University of Louisville

ThinkIR: The University of Louisville's Institutional Repository

$5-2018$

\title{
Investigation of cardiovascular tissue following 56Fe-radiation and potential countermeasure effectiveness.
}

Alexandra R DeCarlo

University of Louisville

Follow this and additional works at: https://ir.library.louisville.edu/etd

Part of the Molecular, Cellular, and Tissue Engineering Commons

\section{Recommended Citation}

DeCarlo, Alexandra R, "Investigation of cardiovascular tissue following 56Fe-radiation and potential countermeasure effectiveness." (2018). Electronic Theses and Dissertations. Paper 2887.

https://doi.org/10.18297/etd/2887

This Master's Thesis is brought to you for free and open access by ThinkIR: The University of Louisville's Institutional Repository. It has been accepted for inclusion in Electronic Theses and Dissertations by an authorized administrator of ThinkIR: The University of Louisville's Institutional Repository. This title appears here courtesy of the author, who has retained all other copyrights. For more information, please contact thinkir@louisville.edu. 
INVESTIGATION OF CARDIOVASCULAR TISSUE FOLLOWING 56FERADIATION AND POTENTIAL COUNTERMEASURE EFFECTIVENESS

\author{
By \\ Alexandra DeCarlo \\ B.S., University of Louisville, 2016

\begin{abstract}
A Thesis
Submitted to the Faculty of the

University of Louisville

J.B. Speed School of Engineering

as Partial Fulfillment of the Requirements

for the Professional Degree
\end{abstract}

MASTER OF ENGINEERING

Department of Bioengineering

April 2018 
INVESTIGATION OF CARDIOVASCULAR TISSUE FOLLOWING 56FERADIATION EXPOSURE AND POTENTIAL COUNTERMEASURES AGAINST RADIATION EFFECTS

Submitted by:

\section{Alexandra DeCarlo}

A Thesis Approved On

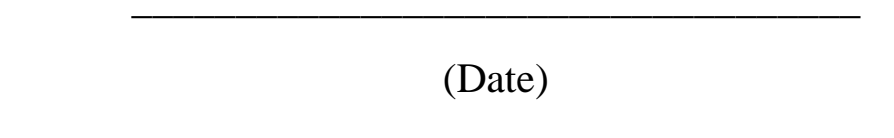

By the Following Reading and Examination Committee:

Dr. Patricia Soucy, Thesis Director

Dr. Kevin Soucy

Dr. Erin Gerber 


\section{ACKNOWLEDGEMENTS}

Thank you to my thesis director, Dr. Patricia Soucy for her guidance and exceptional mentorship as well as the opportunity to work in her lab. Thank you to Betty Nunn, for all of her help in understanding how to work in the lab. Thank you to Dr. Kevin Soucy for his guidance. Thank you to Nick Allen and Larissa Pack for both their assistance with my thesis and for being amazing office mates. Thank you to the members of my thesis committee: Dr. Patricia Soucy, Dr. Kevin Soucy, and Dr. Erin Gerber. Thank you to Dr. Martin O’Toole, Ishita Jain, Rayeanne Balgemann, Rajat Chauhan, Curtis James, Morgan Sharpe, and Agharnan Gandhi as well. Thank you to the University of Louisville's Department of Bioengineering and J.B. Speed School of Engineering for an interesting five years as a student of the University of Louisville. Thank you to my friends and family for their encouragement, patience, and support.

Finally, this work was made possible by the funding support from NASA grant number NNX13AD33A 


\begin{abstract}
Exposure to ionizing radiation is an important medical concern because it can lead to health problems including cancer and cardiovascular issues. In space, astronauts are exposed to ionizing radiation that is not experienced by those who remain on Earth. This radiation can cause health problems such as cardiovascular disease. One way this can happen is through the creation of reactive oxygen species, which can activate TGF- $\beta 1$, contributing to fibrosis or other cardiovascular problems. Antioxidants can be a potential pharmacological mitigator of these excess reactive oxygen species because of their ability to neutralize reactive oxygen species. One such antioxidant with potential as a mitigator for radiation induced cell and tissue damage is curcumin, but is quickly excreted by the body because it is a hydrophobic molecule with a short half-life. In order to retain therapeutically relevant curcumin levels in the body, curcumin needs to be delivered by a drug delivery vehicle. The work presented here examines mice irradiated with ${ }^{56} \mathrm{Fe}$ radiation, to mimic space radiation. These mice were treated with a drug delivery vehicle containing curcumin or a saline sham prior to radiation. Two weeks after radiation, the mice were sacrificed and the hearts and aortas were studied. To examine the effects of radiation on the mouse cardiovascular system, the tissues were evaluated with an aortic outgrowth assay, ROS assay, Masson's trichrome staining, hematoxylin and eosin staining, and wall thickness studies. Statistically, the only significant tissue changes from ${ }^{56} \mathrm{Fe}$ radiation were noted in the thickening of the right ventricle and in the reduction of cardiac muscle per nuclei in the hematoxylin and eosin staining also in the right ventricle. The only significant results of the drug treatments were in the reduction of thinning of the left ventricle by the Alb/CCM and CCM treatments. There were trends of thinning of the
\end{abstract}


left ventricle and thickening of the aortas as a results of the ${ }^{56} \mathrm{Fe}$ radiation. There were drug treatment trends with Alb/CCM leading to the largest outgrowth of the aorta and all treatments leading to a reduced thickening of the right ventricle. 


\section{Table of Contents}

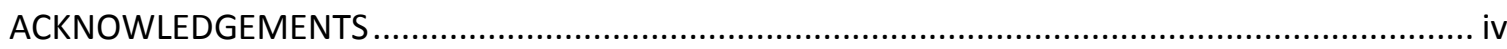

ABSTRACT

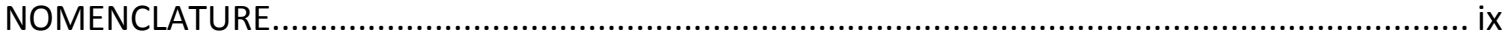

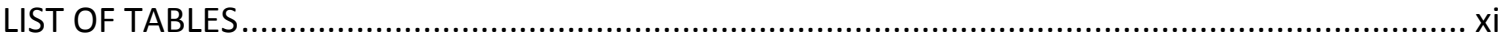

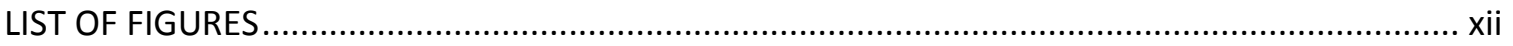

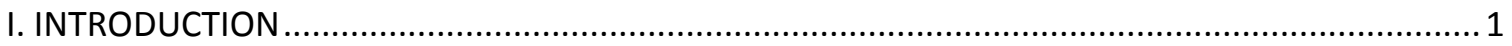

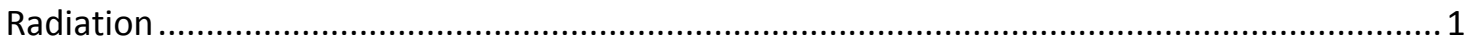

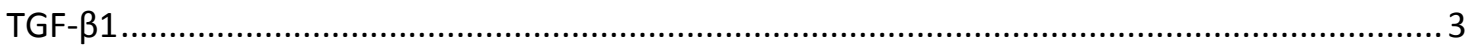

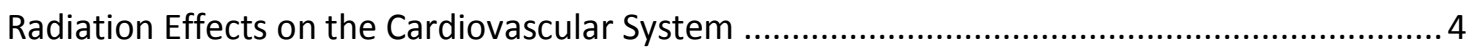

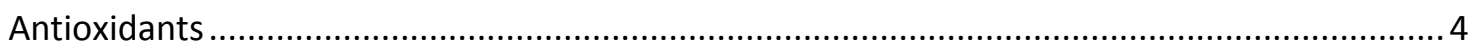

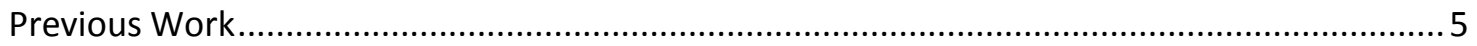

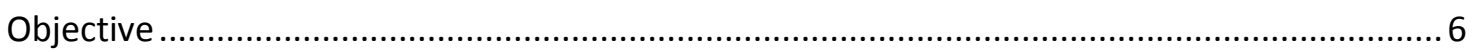

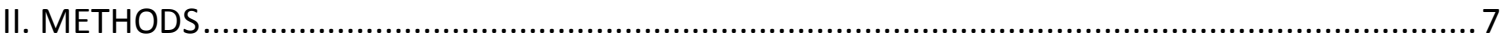

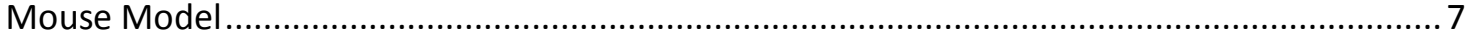

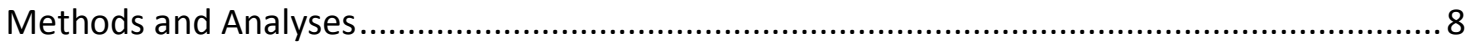

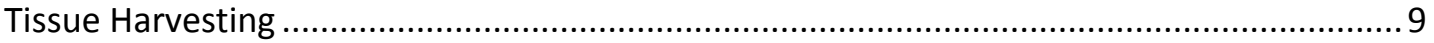

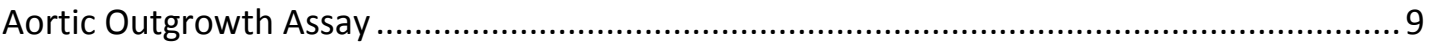

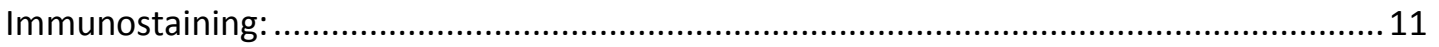

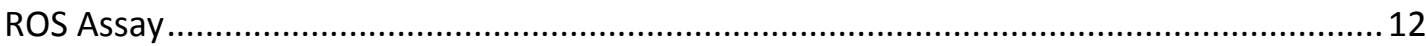

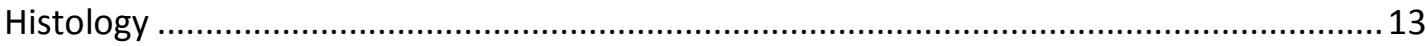

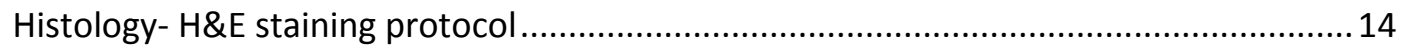

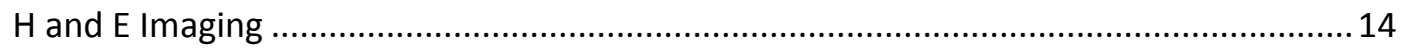

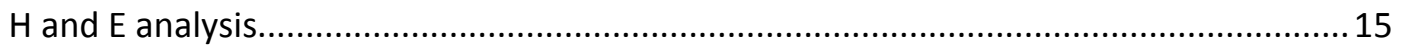

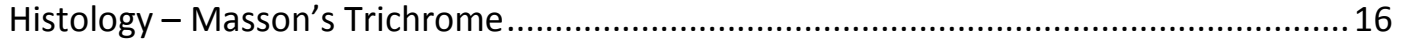

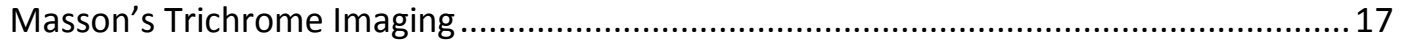

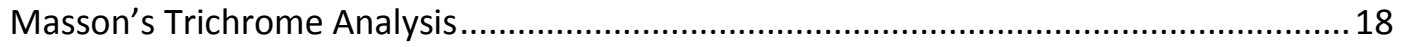

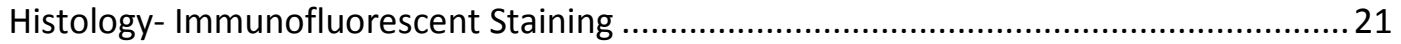

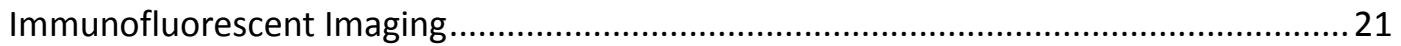

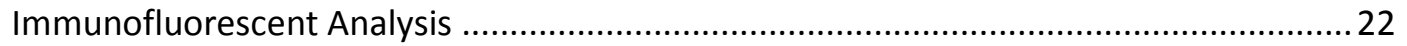

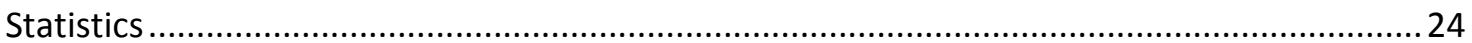

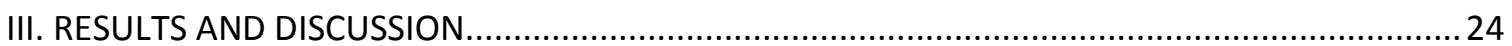

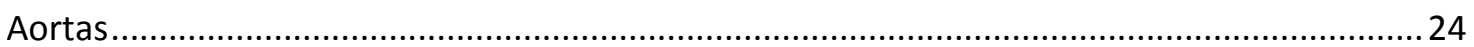




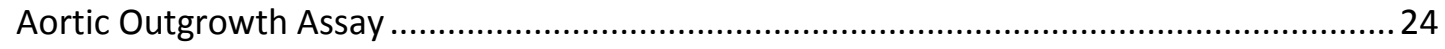

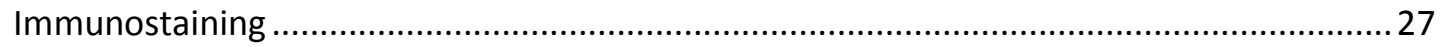

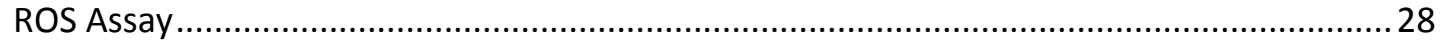

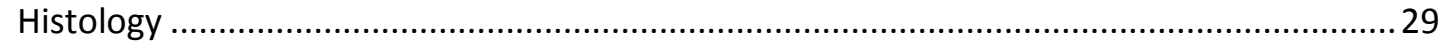

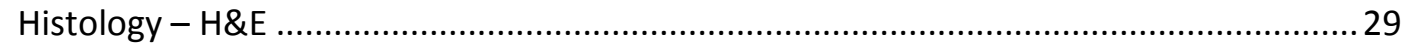

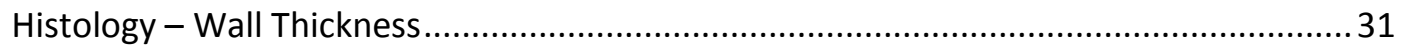

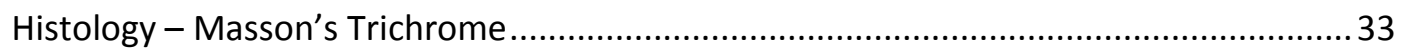

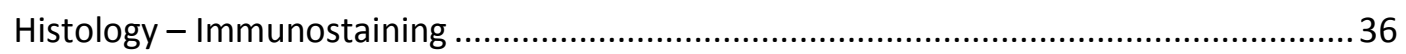

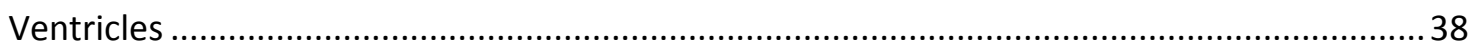

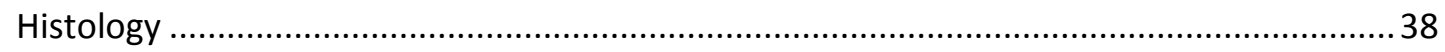

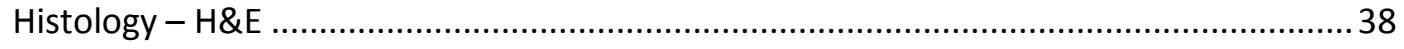

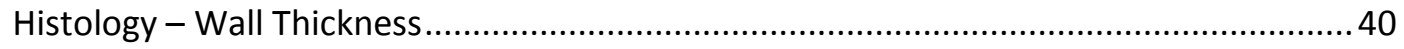

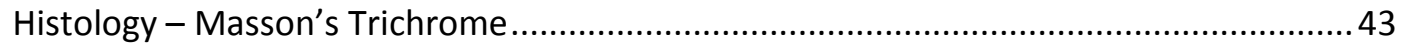

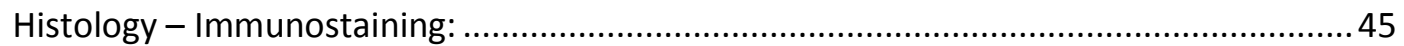

IV. CONCLUSIONS, DISCUSSION, \& RECOMMENDATIONS FOR FUTURE WORK ........................... 48

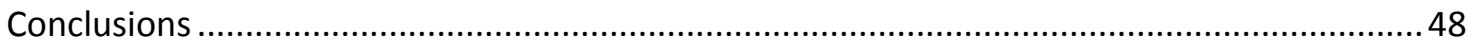

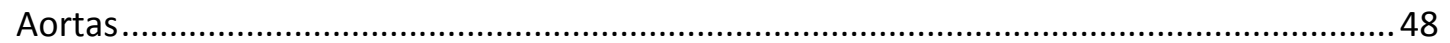

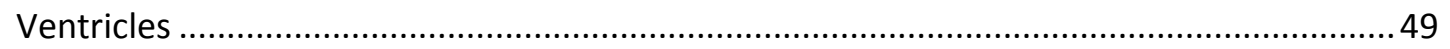

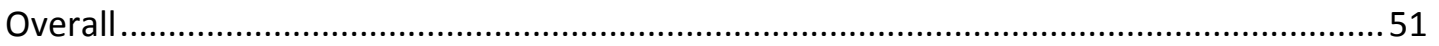

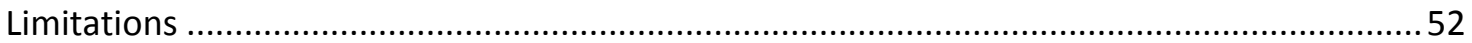

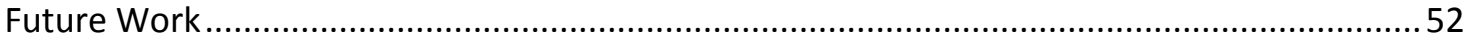

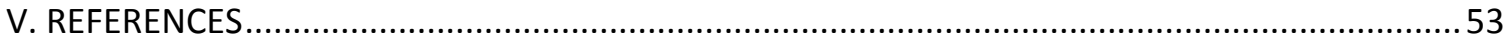

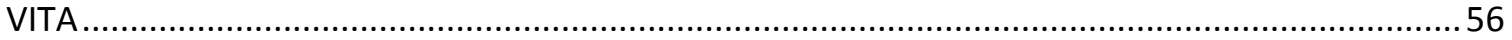

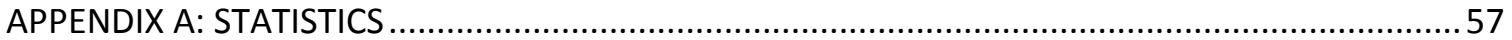


GCR: Galactic Cosmic Radiation

HZE: High Charged Energy

Sv: Sievert

mSv: milliSeivert

Gy: Gray

cGy: centiGray

SPE: Solar Particle Events

ROS: Reactive Oxygen Species

ATP: Adenosine triphosphate

DNA: deoxyribonucleic acid

TGF- $\beta$ : Transforming Growth Factor Beta

TGF- $\beta 1$ : Transforming growth factor beta 1

ECM: Extracellular Matrix

$\mu \mathrm{L}:$ Microliter

$\mu \mathrm{m}$ : micrometer

NR Sal: Non-radiated Saline

Rad Sal: Radiated Saline

Chitocurc: Chitosan-curcumin

Lipids: lipids containing curcumin

Alb/CCM: Albumin-curcumin

CCM: curcumin

$\mathrm{MeV} / \mathrm{n}$ : Megaelectron volt per nucleon

PDMS: polydimethylsiloxane

${ }^{\circ} \mathrm{C}$ : degrees Celsius

PBS: Phosphate buffered Saline

IB4: Isolectin-B4- AF488

BSA: bovine serum albumin

DAPI: 4',6-diamino-2-phenylindole

RIPA: Radioimmunoprecipitation assay 
$\mu \mathrm{g}$ : micrograms

$\mathrm{ml}$ : microliters

mM: millimolar

EDTA: Ethylenediaminetetraacetic acid

TBA: thiobarbituric acid

MDA: 3,4-Methylenedioxyamphetamine

$\mathrm{nm}$ : nanometers

$\mathrm{H}$ and $\mathrm{E}$ : hematoxylin and eosin $(\mathrm{H}$ and $\mathrm{E})$

DI: Deionized

RO: reverse osmosis 


\section{$\underline{\text { LIST OF TABLES }}$}

TABLE 1: Table of the different test groups of mice

TABLE 2: Curcumin delivery and release from delivery vehicles

TABLE 3: Number of mice in each group for aortic outgrowth assay

TABLE 4: Number of mice in each group for ROS assay

TABLE 5: Number of mice in each group for H\&E staining

TABLE 6: Number of mice in each group for Masson's Trichrome staining

TABLE 7: Number of mice in each group for Immunofluorescent staining 


\section{$\underline{\text { LIST OF FIGURES }}$}

FIGURE 1: ImageJ analysis of aortic outgrowth

FIGURE 2: H\&E staining analysis

FIGURE 3: Masson's trichrome imaging

FIGURE 4: Quantification of TGF- $\beta 1$ in immunofluorescent images

FIGURE 5: Aortic outgrowth quantification graph

FIGURE 6: Representative images for aortic outgrowth assay

FIGURE 7: IB4 immunostained aorta

FIGURE 8: Graph of the ROS assay

FIGURE 9: Representative images for the amount of smooth muscle per nuclei in the aortas

FIGURE 10: Graph of the averages and standard deviations for the smooth muscle per nuclei measured in $\mu \mathrm{m}$ in the H\&E stained aortas

FIGURE 11: Representative images for the wall thickness of the aorta

FIGURE 12: Graph of the wall thicknesses of the H\&E stained aortas

FIGURE 13: Graph of the wall thicknesses of each layer of the aorta stained with Masson's trichrome

FIGURE 14: Graph of the adventitia thickness divided by the total aorta thickness for each group

FIGURE 15: Representative images of wall thicknesses of aortas stained with Masson's trichrome

FIGURE 16: Graph of the \% of the total aorta area that is adventitia and the amount of the total aorta area that is collagen.

FIGURE 17: Representative images for the immunostained histology aorta slides

FIGURE 18: Averages and standard deviations for immunostained aortas for both the adventitia and smooth muscle

FIGURE 19: Representative Images for the amount of cardiac muscle per nuclei in the right ventricle

FIGURE 20: Representative Images for the amount of cardiac muscle per nuclei in the left ventricle 
FIGURE 21: Graphs of the averages and standard deviations for the cardiac muscle in $\mu \mathrm{m}$ per nuclei in the H\&E stained right and left ventricles

FIGURE 22: Representative images for the wall thickness of the right ventricle FIGURE 23: Representative images for the wall thickness of the left ventricle

FIGURE 24: Graph of the wall thicknesses of the right and left ventricle as measured from the H\&E stained slides

FIGURE 25: Representative images of the collagen amount found in the left ventricle of the hearts

FIGURE 26: Graph of the amount of collagen found in the Masson's trichrome stained heart

FIGURE 27: Representative images for the TGF- $\beta 1$ intensity of the right ventricle.

FIGURE 28: Representative images for the TGF- $\beta 1$ intensity of the left ventricle FIGURE 29: Graph of the intensity of TGF- $\beta 1$ in the immunofluorescent stained heart. 
Radiation:

\section{INTRODUCTION}

Exposure to radiation can occur with medical treatment such as radiotherapy for breast cancer or Hodgkin's lymphoma [1-4], in areas of occupational exposure such as nuclear or radiation workers [5, 6], or during manned space flight, such as Moon or Mars missions[7-9]. When astronauts are on missions that involve leaving the protection from the Earth's magnetosphere, they are exposed to higher levels of ionizing radiation [10].The high energy type of ionizing radiation is more dangerous than non-ionizing radiation and can result in increased risks of cancer and DNA damage as well as cardiovascular problems [10]. . Ionizing radiation includes gamma rays, $\mathrm{x}$-rays, and galactic cosmic radiation (GCR), which is common during spaceflight. About $1 \%$ of GCR are a type of highly charged ion called High Charged Energy (HZE) particles, which can be of many elements such as carbon, iron, or nickel nuclei. Of these HZE particles seen in space, iron, ${ }^{56} \mathrm{Fe}$ ions, give the largest dose equivalent, which is an expression of radiation dose in terms of biological effect based on the effect of gamma radiation. Thus, ${ }^{56} \mathrm{Fe}$ radiation is the most important biologically and important to study for understanding spaceflight radiation exposure[11]. Since there are many different types of radiation varying

degrees of biological damage, dose equivalent is used to represent the damage and is measured in Sievert (Sv). Conversely, the absorbed dose of radiation is measured in Gray (Gy). Of the GCR, HZE particles are the most dangerous, since they have the strongest ability to pass thorough matter and ionize atoms as they pass. There are also risks of solar particle events (SPE) during space travel [8]. NASA calculated the radiation dose for astronauts as they leave the Earth's magnetosphere. For the Apollo 14 mission ( 9 day mission to the moon) the radiation dose was $11.4 \mathrm{mSv}$, while for a 3 year Mars mission, the estimated radiation dose would be $1200 \mathrm{mSv}$. 
There are also possibilities of solar events which would increase the radiation dose[12]. The NASA career exposure limit for astronauts is between 1 and $4 \mathrm{~Sv}$ depending on age and gender, with younger people having lower limits since they are likely to be exposed to more radiation through the rest of their lives[10]. The Mars mission alone would surpass the astronaut's career exposure limit of 1 . The career exposure limit is based upon a maximum $3 \%$ increase in risk of cancer mortality. The longer the mission and the deeper into space the astronauts go, the more important it is to develop proper protection for the astronauts' health from radiation exposure.

The main ways to reduce exposure to radiation from manned space missions are by limiting astronaut time in space and using shielding[13, 14]. Future missions to Mars will extend the tine in space instead of limiting the time. In addition, only so much shielding can be added to the spaceship due to weight and cost concerns for the spaceship. Another option is a pharmacological approach to minimize damage to the cells and tissue from the radiation exposure. If the exposure cannot be prevented, minimizing the resultant damage is a promising approach.

Understanding molecular changes in cells can aid in the development of drugs to mitigate radiation damage. Ionizing radiation is known to cause reactive oxygen species (ROS), which are chemically reactive molecules (free radicals) that contain oxygen such as superoxide anions, hydroxyl radicals, and hydrogen peroxide. ROS serve as signaling molecules under normal physiological conditions [15]. Intracellular ROS are produced largely by the mitochondria as the mitochondria use oxygen to produce ATP. The main ROS produced by mitochondria is

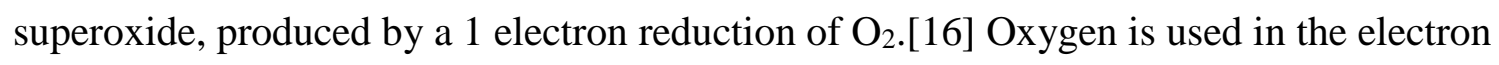
transport chain, as oxygen in reduced by electrons donated from NADH and FADH2 and then turned to water. If the electrons leak out early to oxygen, superoxide is formed [17]. This usually 
occurs at complex I or complex III. Whether or not superoxide is produced and how quickly depends on concentrations of enzyme containing electron carriers that are transiently in a redox form and able to react with $\mathrm{O}_{2}$. [16] If there is a high $\mathrm{NADH} / \mathrm{NAD}^{+}$ratio, it can cause superoxide production in complex 1 due to electron leakage. If there is a reduced CoQ pool and $\mathrm{Q}_{i}$ site inhibited by antimycin, superoxide production occurs in complex III [16]

There are also ROS generated by phagocytic cells to fight off infection. ROS can also serve as second messengers in signal transduction pathways in the body[18]. A rise in ROS levels leads to cellular damage as well as the activation of signaling pathways and has been linked to diseases such as atherosclerosis. There is a natural antioxidant defense system of the body that scavenges excessive ROS. The ROS and antioxidant levels stay in a balance, but if the formation of ROS outpaces the degradation by antioxidants, oxidative stress will result. Exposure to radiation increases ROS levels, causing DNA damage as well as long term effects on mitochondrial functions, and results in activation of metabolic pathways [19]. Along with the general damage to the cells that ROS can cause, ROS can activate other biologically active molecules such as growth factors like TGF- $\beta$, which can lead to fibrosis of tissues [20].

\section{$\underline{\text { TGF- } \beta 1:}$}

Transforming growth factor beta 1 (TGF- $\beta 1)$ is a cytokine mostly implicated in wound healing and in fibrosis [21]. TGF- $\beta 1$ has a role in the activation of fibroblast, promotion of fibrosis, and formation of scar tissue [22]. Others have demonstrated radiation increased ROS and subsequent activation of TGF- $\beta 1$, in mouse bone marrow and mammary glands and in cells $[23,24]$. Excess TGF- $\beta 1$ is linked to progressive fibrosis via the deposition of collagen and other extracellular matrix (ECM) components. TGF- $\beta 1$ can directly affect the health of the cardiovascular system, causing excess ECM production in the heart and arteries [25]. 


\section{$\underline{\text { Radiation Effects on the Cardiovascular System: }}$}

One system affected by ionizing radiation is the cardiovascular system $[3,5,7,26]$ with an increased risk of myocardial infarction, coronary heart disease, and atherosclerosis[3]. Both gamma radiation (at doses of $0,50,160$, and $500 \mathrm{cGy}$ ) and $\mathrm{HZE}{ }^{56} \mathrm{Fe}$ radiation (at doses of 0 , 0.5 , and $1 \mathrm{~Gy}$ ) have been shown to cause impaired angiogenesis in rats, which indicated poor vascular health [11,27]. Radiation exposure also increased the stiffness of the blood vessels resulting in increased blood pressure, decreased endothelial function, and atherosclerosis [27]. Terrestrial ionizing radiation from radiotherapy has also shown vessel thickening in clinical settings[28]. Biopsies were taken of arteries at a mean of 4 years after irradiation (with a range of 3 months to 26 years) from patients undergoing free-flap reconstruction surgery, and the thickening of the intima was found by comparing the intima thickness to the media thickness. The ratio of intima thickness to media thickness was larger in cases of radiation, with the largest effects observed in those receiving the surgery longer after the initial radiation [28]. The main tissue protein related to stiffness is collagen, while the protein most related to flexibility in tissue is elastin. The levels of these proteins help determine the overall mechanical properties of the arteries or heart. For example, in mice with cardiomyopathy, ventricular thickening was shown to be due to oxidative stress, which resulted in higher expression of fibrosis markers and of collagen [29].

\section{Antioxidants:}

The body's natural defense against ROS is antioxidants, which can be produced by cells or found in external sources. One antioxidant is curcumin, derived from the spice turmeric and shown to have anti-carcinogenic effects, anti-proliferative effects, antioxidant effects, antiinflammatory effects, and anti-angiogenic effects as well as an ability to repair DNA [30-32]. Curcumin has the ability to reduce oxidative stress, as well as to inhibit the transcription of genes 
that relates to inflammatory responses and modulate the paths of growth factors such as TGF- $\beta 1$ [33]. Curcumin has been shown to be non-toxic at up to 8g/day in humans [34]. However curcumin is unstable with degradation rates dependent on $\mathrm{pH}$ [30]. Given its hydrophobicity, curcumin has poor update in the body when administered orally. In addition, orally administered curcumin is quickly metabolized and excreted in the urine and feces. Intravenous administration of curcumin is quickly removed in the bile [30]. Thus, curcumin has strong potential as an antioxidant for mitigation of radiation induced damage, but it has low bioavailability in vivo.

Nanoparticles have been shown as a solution to enhance the bioavailability curcumin [31, 35]. A curcumin-chitosan polymer was able to stay stable in water for over 30 days as compared to the 30 minutes that would occur with curcumin alone [35]. Phospholipid vehicles have been shown to extend the bioavailability of orally delivered curcumin in mice. The maximum concentration of curcumin was increased from $266.70 \mathrm{ng} / \mathrm{ml}$ to $600.93 \mathrm{ng} / \mathrm{ml}$ and the duration of the curcumin increased from 1.62 hours to 2.33 hours [36]. Albumin nanoparticles have also been tested for delivery of curcumin, with resulting higher accumulation in tissue and detectable amounts of curcumin retained in the tissue after 4 hours [37].

\section{Previous Work:}

Our group has fabricated three novel drug delivery systems for curcumin: albumin, lipids, and chitosan. Albumin, in this case Human Serum Albumin, is the main protein of human blood plasma. Albumin is a common drug delivery vehicle and has been shown to bind to curcumin, as have lipids and chitosan $[35,38,39]$. Lipids are biological substances including fats that are soluble in nonpolar solvents. Chitosan is a polysaccharide made by treating chitin from crustacean shells with an alkaline. Chitosan was bonded covalently to curcumin to make a product called Chitocurc which provided a burst release after exposure to radiation [35]. 
Albumin, fusogenic lipids, and chitosan all release curcumin at various rates: lipids-minutes, albumin-hours, chitosan-days. The specific release times are provided in Table 2.

A mouse model was developed to test the three nanoparticle drug delivery vehicles, during which the mice the nanoparticles were injected via the tail vein and then the mice were exposed to 0 or $4 \mathrm{~Gy}$ cesium- $137 \gamma$ radiation. Ten days later the mice were sacrificed and the tissues were harvested. Each of the three drug delivery vehicles demonstrated some protective effects. ROS in bone marrow was higher in irradiated mice than non-irradiated. The mice receiving particle treatments still had increased ROS compared to non-irradiated mice, but ROS levels were less than irradiated mice treated with saline. The treatment with the lowest ROS was Chitocurc. Radiation also damaged hematopoietic progenitor cells, and the lipid vesicle treatment provided the most protection as measured by increased number of bone marrow colonies. Finally, radiation resulted in a near 50\% reduction in endothelial cell outgrowth from aortas, a marker of endothelium damage. While the treatments could not fully prevent the endothelium damage, lipid vesicles showed more outgrowth than the radiated saline.

\section{Objective:}

The goal of this work was to assess the curcumin nanoparticles' effectiveness at reducing the ${ }^{56} \mathrm{Fe}$ radiation induced cardiovascular pathologies. We hypothesized that mice treated with the curcumin nanoparticles will exhibit reduced ROS in the cardiovascular tissue, reduced tissue remodeling, and reduced endothelial cell damage in response to radiation exposure. To achieve this, two aims were addressed.

Aim 1: Characterize the effect of ${ }^{56} \mathrm{Fe}$ radiation on mouse cardiovascular tissue (heart and aorta). 
Aim 2: Examine the effectiveness of three independent curcumin drug delivery mechanisms at reducing mouse cardiovascular tissue damage caused by ${ }^{56} \mathrm{Fe}$ radiation.

\section{METHODS}

Mouse Model:

The overall experimental design involved a post-test only design on 54 wild type mice (C57BL/6J 6 month old males) because the assessment of the cardiovascular system following radiation exposure required sacrifice of the mice. There were a total of 6 groups of mice: 1 . Injected with saline but not irradiated (NR Saline), 2. Injected with saline and irradiated (Rad Saline), 3. Injected with curcumin without a drug delivery vehicle and irradiated (CCM), 4. Injected with lipid drug delivery vehicle with CCM and irradiated (Lipids), 5. Injected with albumin drug delivery vehicle with $\mathrm{CCM}$ and irradiated (Alb/CCM), and 6. Injected with chitosan drug delivery vehicle with CCM and irradiated (Chitocurc). The number of mice given each treatment is listed in Table 1 below. The amounts of curcumin delivered with each drug delivery vehicle and their release times are listed below in Table 2. All studies carried out followed procedures approved by the University of Louisville and Brookhaven National Labs IACUCs. 
TABLE 1: Table of the different test groups of mice.

\begin{tabular}{|l|l|l|l|}
\hline Contents Injected & Radiation (yes/no) & $\begin{array}{l}\text { Treatment } \\
\text { Abbreviation }\end{array}$ & Number of mice \\
\hline Saline & No & NR Sal & 9 \\
\hline Saline & Yes & Rad Sal & 10 \\
\hline $\begin{array}{l}\text { Chitosan with } \\
\text { curcumin }\end{array}$ & Yes & Chitocurc & 9 \\
\hline Lipids with curcumin & Yes & Lipids & 10 \\
\hline $\begin{array}{l}\text { Albumin with } \\
\text { curcumin }\end{array}$ & Yes & Alb/CCM & 10 \\
\hline Curcumin & Yes & CCM & 6 \\
\hline
\end{tabular}

TABLE 2: Curcumin release from delivery vehicles

\begin{tabular}{|l|l|l|}
\hline Delivery Vehicle & CCM Release in $\mu \mathrm{g} / \mathrm{kg}$ & $100 \%$ release time in vitro \\
\hline Lipids & 121 & $15 \mathrm{~min}$ \\
\hline Alb/CCM & 252 & $60-90 \mathrm{~min}$ \\
\hline Chitocurc & 6900 & 19 days \\
\hline CCM & 273 & Immediate \\
\hline
\end{tabular}

Methods and Analyses:

Radiation:

Mice (6 months old and of type C57BL/6J) are common animals used in high energy radiation models [40-42]. Each of the mice was injected in the tail vein with either one of the drug delivery mechanisms or saline. All groups except for the non-radiated saline (NR Sal) treatment group were exposed to $20 \mathrm{cGy}$ of $1000 \mathrm{MeV} / \mathrm{n}{ }^{56} \mathrm{Fe}$ at $20 \mathrm{cGy} / \mathrm{min}$ at Brookhaven National Labs. Two weeks (14 days \pm 2 days) following radiation the mice were sacrificed. The two week time point was selected to allow for transport back to UofL and minimize any possible 
effects from transportation. For this particular project, the hearts and aortas were harvested to examine the effects of the radiation and the drug delivery vehicles.

\section{Tissue Harvesting:}

The hearts and aortas were kept together when dissected from the mice, and immediately placed in endothelial growth media without serum (medium 200). The connective tissue was carefully removed from the aorta and heart under a dissecting scope. Briefly the heart and aorta were pinned to a layer of PDMS in a petri dish and covered in endothelial growth media for the cleaning process. A set of Vannas spring scissors was used to gently pull the connective tissue away without damaging the heart or aorta. Then the heart and aorta were cut so that they were no longer connected to each other. Each aorta was flushed with endothelial growth media to remove blood. Using a scalpel, the heart was initially cut into three cross-sections containing: the atria, the ventricles, and the apical ventricles.

\section{Aortic Outgrowth Assay:}

The aortic outgrowth assay was adapted from the method used by K. Soucy et al [40]. A one centimeter cross-section was cut from the center of each aorta with a scalpel for the aortic outgrowth assay. This one centimeter section was cut into four equal pieces with a scalpel, three of which were used in aortic outgrowth assay, so that each aorta could be tested in triplicate. The number of mice used from each treatment group are listed in Table 3. The assay was a Matrigel matrix endothelial cell outgrowth assay utilizing Matrigel (BD Biosciences) diluted 1:1 with endothelial cell outgrowth assay with serum (Medium 200 phenol red free). The diluted Matrigel solution $(150 \mu \mathrm{L})$ was added to each well of a sterile 48 -well cell culture plate with care to minimize bubbles and incubated at $37^{\circ} \mathrm{C}$ for at least 30 minutes to cure. One ring of aorta was gently placed with tweezers in the center of each well with the lumen of the aorta oriented 
horizontally. Then the aortas were covered with $100 \mu \mathrm{L}$ of diluted Matrigel to fix the aorta in place. The samples were incubated for 4 days and then the cell outgrowth from each end of the aorta was imaged using the 4x objective on a light microscope (Nikon Eclipse TE2000-U, Japan). The image was taken using the focus plane that displayed the greatest number of cellular outgrowth.

TABLE 3: Number of mice in each group for aortic outgrowth assay.

\begin{tabular}{|l|l|}
\hline Treatment & Number of mice \\
\hline NR Saline & 5 \\
\hline Rad Saline & 7 \\
\hline Chitocurc & 6 \\
\hline Lipids & 6 \\
\hline Alb/CCM & 6 \\
\hline CCM & 6 \\
\hline
\end{tabular}

NIH ImageJ software was used to quantify aortic outgrowth. Only one image per aorta slice was quantified, resulting in a total of three images per mouse, one from each well. The end of the aorta with the most cell growth from each slice was selected for imaging and quantification. Each image was opened in ImageJ twice so that one was used for quantification and the other used for comparison during the analysis. A custom macro was run to convert the image to 8-bit and set the scale (pixel to micron ratio) as well as to run the functions "background correction" and "unsharp mask" in ImageJ. The resultant image is shown in Figure 1B. Then the image was given a threshold using the Threshold command as shown in Figure 1C to filter out any selections that are not outgrowth. The automatic threshold was accepted unless it resulted in non-outgrowth selections, in which case the threshold was adjusted until only cell 
outgrowth was selected. Then the figure was converted to binary (Figure $1 \mathrm{C}$ below) in order to make a monochromatic image. A polygon was then drawn around the outgrowth using the Freehand Selection tool to separate the outgrowth from the aorta (Figure 1E). The measurement of the outgrowth within the polygon area was taken, allowing for quantification of the total area of the outgrowth from each aorta.

A

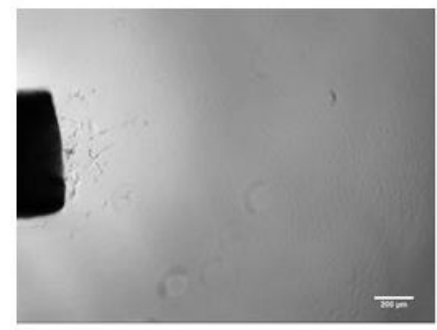

D

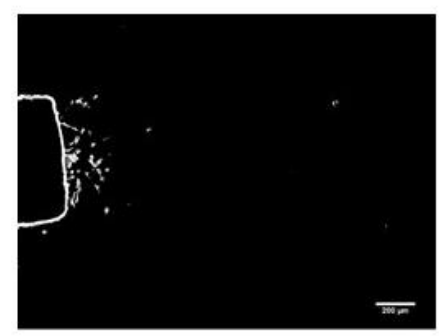

B

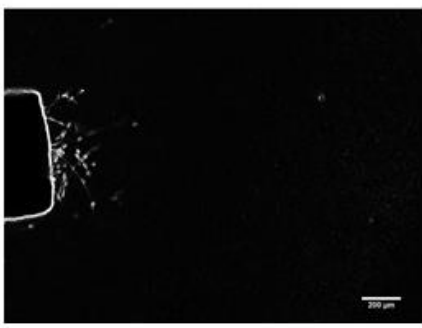

$\mathrm{E}$

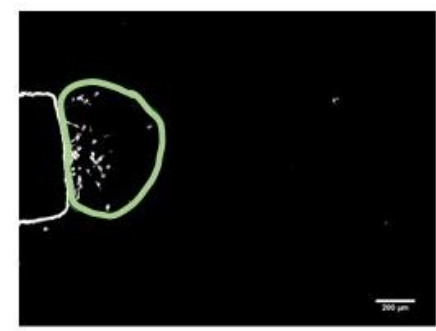

C

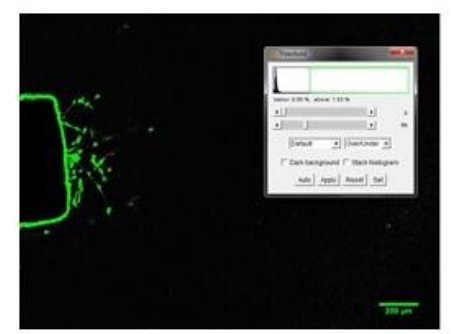

FIGURE 1: Image J Analysis of Aortic Outgrowth. All images with scale bar of $50 \mu \mathrm{m}$. A. Original image of the aorta. B. Outgrowth quantification macro applied. C. Thresholded image. D. Image converted to Binary. E. Freehand selection of the outgrowth area.

Immunostaining:

Immunostaining was done on the outgrowth assay aortas to ensure that the cells quantified were endothelial cells. Immediately after the light images described above were captured, the samples were fixed with $4 \%$ formaldehyde for 1 hour. The samples were stored in $1 \mathrm{X} P B S$ in the refrigerator. 
Isolectin-B4- AF488 (Invitrogen) is an antibody that stains endothelial cells specifically. Isolectin-B4- AF488 (IB4) was diluted to $1: 1000$ in $0.1 \%$ bovine serum albumin (BSA) and added to the samples, which were incubated for 2 hours at 37C. Samples were washed four times with PBS at room temperature for 10 minutes each. DAPI (SIGMA-Aldrich) at $30 \mu \mathrm{M}$ in $0.1 \%$ BSA was used to stain the nuclei in the sample for 15 minutes at $37^{\circ} \mathrm{C}$. Samples were washed 6 times with PBS and then imaged with fresh PBS.

The immunostained aortas were visualized on an epifluorescent microscope with a fluorescence illumination system (X-Cite 120 from EXCO). A white light image was also taken for the purpose of confirming location. The aortas were captured at 20x magnification using NIS Elements Acquisition program. Three images were taken for each side of the aorta, each on a different focal plane since in Matrigel the cells were in a three dimensional matrix and so did not all grow in one plane. The violet filter with an excitation wavelength range between 379 and 420 on the fluorescent microscope was used to locate viable nuclei that had been stained with DAPI. Then the white light filter was used for an overall view before the blue light filter with an excitation wavelength range between 420 and 495 was used to identify the endothelial cells stained with IB4. The IB4, white light, and DAPI images were taken without changing the focus so that the images could be merged.

\section{ROS Assay:}

The remaining aorta pieces from the mice shown in Table 4 below were weighed and solubilized in RIPA buffer(Cayman Chemicals) with $10 \mu \mathrm{g} / \mathrm{ml}$ leupeptin and $1 \mathrm{mM}$ EDTA and then homogenized with an electric tissue homogenizer (Fisher Scientific, USA) on ice. After homogenization, the samples were be centrifuged at $1600 \mathrm{xg}$ at $4^{\circ} \mathrm{C}$ for 10 minutes, and the supernatants were be collected and frozen at $-80^{\circ} \mathrm{C}$ to be saved for the ROS assay (TBARS 
Assay Kit (Cayman Chemical)). The amount of ROS in the aortas was quantified to assess the cardiovascular damage from the radiation. For the assay $1 \mathrm{~g}$ thiobarbituric acid, $10 \mathrm{~mL}$ acetic acid in $40 \mathrm{~mL}$ water, $10 \mathrm{~mL}$ of $3.5 \mathrm{M}$ sodium hydroxide in $40 \mathrm{~mL}$ water, $1 \mathrm{~mL}$ of $500 \mu \mathrm{M}$ malondialdehyde, and $10 \mathrm{~mL}$ of $10 \%$ trichloroacetic acid was prepared following manufacturer's instructions. To prepare the color reagent, $106 \mathrm{mg}$ of TBA, $10 \mathrm{~mL}$ of the diluted acetic acid, and $10 \mathrm{~mL}$ of the diluted sodium hydroxide were mixed together. For calorimetric measurement, the standards were created according to the TBARS assay kit protocol of standards of MDA of concentrations from $0-50 \mu \mathrm{M}$. After reagent preparation, $100 \mu \mathrm{L}$ of a sample was added to 100 $\mu \mathrm{L}$ trichloroacetic acid and $800 \mu \mathrm{L}$ of the color reagent. Each sample was vortexed and boiled for one hour. Next, the samples were placed in ice for 10 minutes to stop the reaction, then they were centrifuged. Triplicates of each sample were pipetted into wells (200 $\mu \mathrm{L} /$ well) on the 96 well plate. The absorbance was measured at 530-540 $\mathrm{nm}$.

TABLE 4: Number of mice in each group for ROS assay

\begin{tabular}{|l|l|}
\hline Treatment & Number of mice \\
\hline NR Saline & 3 \\
\hline Rad Saline & 3 \\
\hline Chitocurc & 2 \\
\hline Lipids & 3 \\
\hline Alb/CCM & 4 \\
\hline
\end{tabular}

\section{Histology:}

The fourth section of the one centimeter section of aorta was placed in $4 \%$ formaldehyde for 24 hours then switched to $70 \%$ ethanol to later be fixed in paraffin for histology. The ventricles were also placed in $4 \%$ formaldehyde for 24 hours then switched to $70 \%$ ethanol. 
These aortas and ventricles samples were sent to the University of Louisville's Pathology lab to be embedded in paraffin and cross-sectioned into $5 \mu \mathrm{m}$ slices. Four slides containing three tissue sections were made from each paraffin block, with the first being stained with hematoxylin and eosin ( $\mathrm{H}$ and $\mathrm{E}$ stain) and the other three unstained

\section{Histology- H\&E staining protocol:}

$\mathrm{H}$ and $\mathrm{E}$ is stain where hematoxylin stains nuclei while eosin stains eosinophilic structures like proteins. This was done for the aortas in Table 5 below. The stained slides were imaged using an EVOS XL Core Brightfield Microscope.

TABLE 5: Number of mice in each group for H\&E staining.

\begin{tabular}{|l|l|l|}
\hline Treatment & Number of hearts & Number of aortas \\
\hline NR Saline & 5 & 2 \\
\hline Rad Saline & 4 & 3 \\
\hline Chitocurc & 4 & 4 \\
\hline Lipids & 3 & 3 \\
\hline Alb/CCM & 4 & 2 \\
\hline CCM & 4 & 3 \\
\hline
\end{tabular}

\section{$\underline{H}$ and E Imaging:}

For the each cross-section of the ventricles, two images were taken at $4 \mathrm{x}$ to combine into a full view of the heart slice, two images were taken at 40x at different places on the left ventricle, and two images were taken at 40x at different places on the right ventricle. For the aortas, one image was taken at 10x for a full view and two images were taken at different locations on the aorta at 40x. The difference in magnification was due to the difference in tissue size between hearts and aortas. Since the hearts are much larger, a lower magnification was needed to take full images of the heart slices. 


\section{$\underline{\mathrm{H} \text { and } \mathrm{E} \text { analysis: }}$}

Once these images were taken, they were analyzed in ImageJ. For the hearts, thickness of the left and right ventricle walls was quantified to examine for signs of fibrosis or cardiovascular disease. For this analysis, the image was first opened in ImageJ. Then the scale was set to change the distance from pixels to microns based on the magnification from the microscope. Once the image was opened, the Straight tool was used to draw lines across the left ventricle to measure the thickness. This was done at 5 points along each ventricle, and the mean thickness was noted for the left and right ventricles. The same thickness characterization and comparison was also done for the aortas.

The second ImageJ quantification was a red/blue contrast quantification of the 40x ventricle images and the 40x aorta images. $\mathrm{H}$ and $\mathrm{E}$ stains nuclei blue and intracellular and extracellular protein red. There are a variety of shades of red for different proteins, and muscle in particular is stained dark red. Using thresholding techniques in Image $\mathbf{J}$, the area of nuclei and muscle tissue can be quantified. To do this, first the image was opened in ImageJ. Then the scale was set using the Set Scale command to find the measurements in microns. Then, the Color Threshold command was used twice. The first time was to find the percent of the image that was muscle tissue. The Color Threshold command was used, and the scales were adjusted as shown in Figure $2 \mathrm{~B}$ so that only the red hue area was selected as show in Figure $2 \mathrm{C}$. Then the image was analyzed using the Analyze Particles command, choosing the create mask option in the drop down list. This mask is shown in Figure 2D. This process was repeated to select only the nuclei without the muscle layer as shown in Figure 2E. Again the image was analyzed and a mask created, shown below in $2 \mathrm{~F}$. 
A

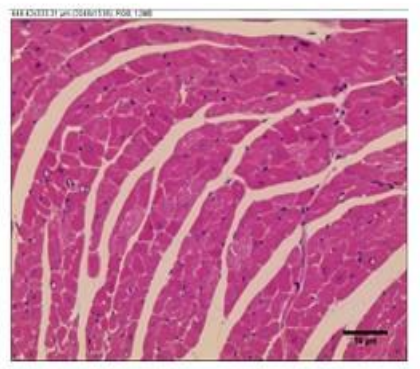

D

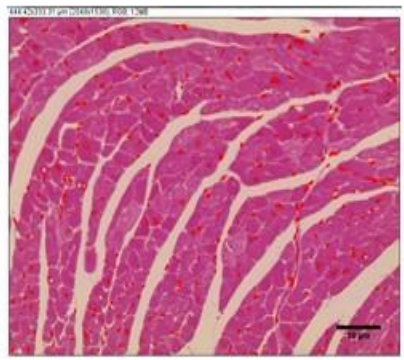

B

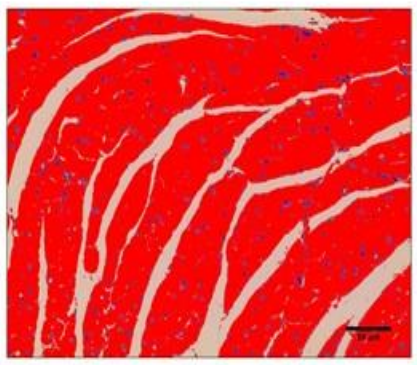

$\mathrm{E}$

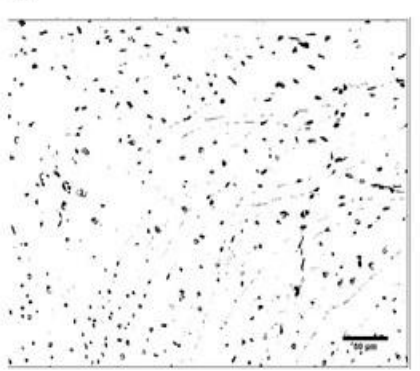

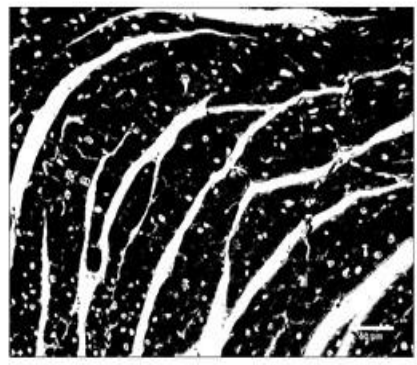

FIGURE 2: H\&E staining analysis with scale bars of $50 \mu \mathrm{m}$. A: the original left ventricle image.
B: muscle tissue selected in red. C: mask of muscle tissue. D: Nuclei selected in red. E: mask of nuclei.

Histology - Masson's Trichrome:

Masson's trichrome staining of the heart and aorta slices was also performed. This was done for one slide of each heart or aorta shown in Table 6 below. The staining for Masson's trichrome was to differentiate between different extracellular matrix components, specifically collagen and elastin. The procedure for Masson's trichrome staining was done with a kit from Sigma-Aldrich (HT15-1KT), by following manufacturer's instructions. Weigerts Iron Hematoxylin solution was made from equal parts of hematoxylin component A and hematoxylin component B (Sigma). The Bouin's solution as poured into a coplin jar that was then heated in the oven until it reached $56^{\circ} \mathrm{C}$. The phosphotungstic/phosphomolybdic acid solution was made with one part phospotungstic, one part phosphomolybdic, and two parts reverse osmosis (RO) water. The paraffin as removed with two five minute washes in xylene, a three minute wash in $100 \%$ ethanol, and three minute wash in $70 \%$ ethanol, and three minute wash in 50\% ethanol, 
and a three minute wash in distilled water. The slides are then placed in warmed Bouin's solution for 15 minutes. Then the slides are placed in tap water for 5 minutes before being rinsed carefully under running RO water. Then the slides were dipped in Weigerts iron hematoxylin solution for 5 minutes before being rinsed in tap water for 5 minutes. Then the slides were placed in Biebrich scarlet-acid fucshin for five minutes followed by another RO water rinse. The slides were then stained with phosphotungstic/phosphomolybdic acid solution for five minutes followed by an aniline blue stain for five minutes. The slides were immediately placed in $1 \%$ acetic acid for 2 minutes then rinsed in $\mathrm{RO}$ water. The slides were then dehydrated with a one minute soak in $95 \%$ ethanol, two one-minute soaks in $100 \%$ ethanol, and two four-minute soaks in xylene. The slides are mounted with Permount and left to dry for 4 days before imaging.

TABLE 6: Number of mice in each group for Masson's Trichrome staining

\begin{tabular}{|l|l|l|}
\hline Treatment & Number of hearts & Number of aortas \\
\hline NR Saline & 3 & 2 \\
\hline Rad Saline & 3 & 3 \\
\hline Chitocurc & 3 & 3 \\
\hline Lipids & 3 & 3 \\
\hline Alb/CCM & 3 & 2 \\
\hline CCM & 3 & 3 \\
\hline
\end{tabular}

\section{Masson's Trichrome Imaging}

The Masson's Trichrome stained hearts and aortas were also imaged on the EVOS XL

Core Brightfield Microscope. For the each slice of heart, two images were taken at $4 \mathrm{x}$ to combine into a full view of the heart slice, two images were taken at 40x at different places on the left ventricle, and two images were taken at 40x at different places on the right ventricle. For 
the aortas, one image was taken at 10x for a full view and two images were taken at different locations on the aorta at 40x.

\section{Masson's Trichrome Analysis}

These images were also analyzed in ImageJ. The scale was set using the Set Scale command to find the measurements in microns. Then, the Color Threshold command was used which has 3 adjustable options: Hue (a specific color tone), Saturation (how vivid a color is), and Brightness (relative lightness or darkness). Each of these options is from 0-255, allowing for 256 possible values of color hue, saturation, and brightness with 8 bits per pixel. The first time, for the selection of the entire aorta, the Hue slider covered the entire range of options from 0-255. Then Saturation was adjusted to eliminate background noise by not selecting the lowest saturation levels. The brightness was selected as 100-255, since this was the point at which all parts of the aorta were selected without any pieces mistaken for background. The image was then converted to binary and measured as shown in Figure 3B. Next, the original reopened and the color Threshold was used again with the Hue slider adjusted so it only selected the red range, generally between 215 or 225 to 255 . This excluded the adventitia and nuclei from quantification. The saturation and brightness were the same as the previous image. The binary image is shown in Figure 3C. The third step was to find the elastin in the media layer. For this, the image was zoomed in on so that the lines of elastin could be seen. The hue slider was moved to exclude the lower levels of red, usually selecting around 248 or $250-255$. The image was then converted to binary and measured as shown in Figure 3D. The next selection was of smooth muscle cells. The bottom of the media layer threshold from the previous measurement in $3 \mathrm{C}$ served as the bottom threshold for the hue. The lower number of the elastin threshold in 3D minus one was the upper threshold for the hue threshold. These measurements would result in a hue range similar to $215-248$. The brightness was made to include the full range of brightness. 
This was then converted to binary and measured as shown in Figure 3E, representing smooth muscle in the media layer. The next selection was of the adventitia layer. The low threshold for the hue was adjusted until as much of the adventitia as selected as possible without other layers being selected, often at around 90 . The high threshold for the adventitia was defined as the low threshold from the media layer (2C) minus one. This image was converted to binary and measured, representing adventitia area (Figure 3F). The final selection was of the collagen in the adventitia. The lower hue threshold was measured by zooming in to the image and selecting until as much of the collagen was selected as possible without other parts of the aorta being selected, similar to the method of selecting elastin. The high threshold of the adventitia starts at the low threshold for the media layer minus one, but may need to be reduced further to eliminate some pink or purple area in the adventitia. The collagen layer in the adventitia was also converted to binary and measured as shown in Figure 3G. The thickness was calculated for the total aorta (2B), the media layer, and the adventitia layer by taking the binary images and measuring the width. 


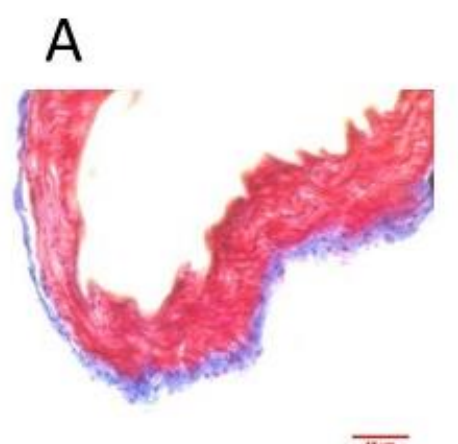

D

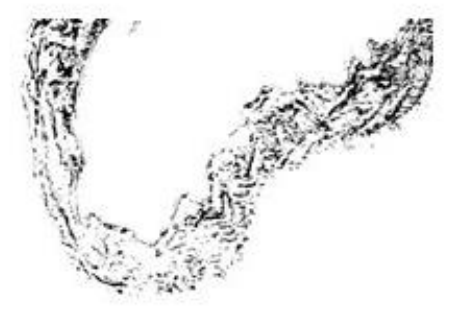

G

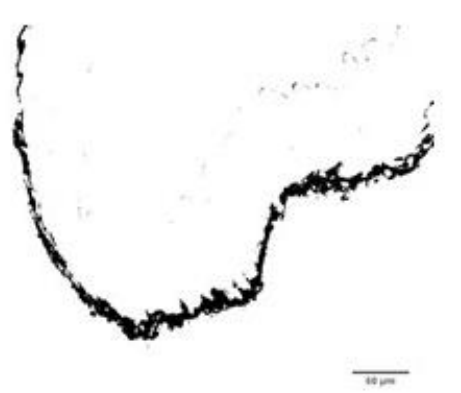

B

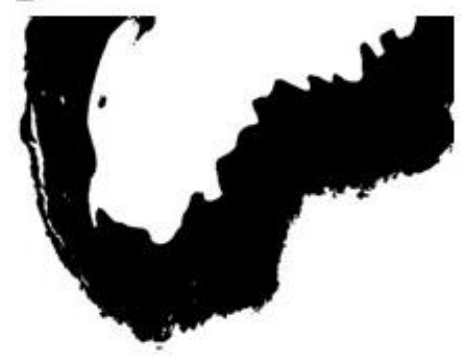

$E$

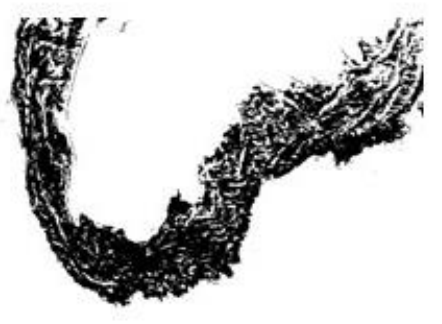

C

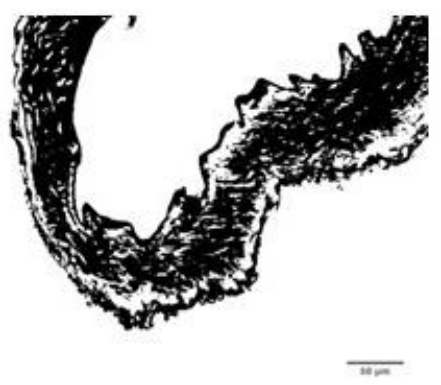

$\mathrm{F}$

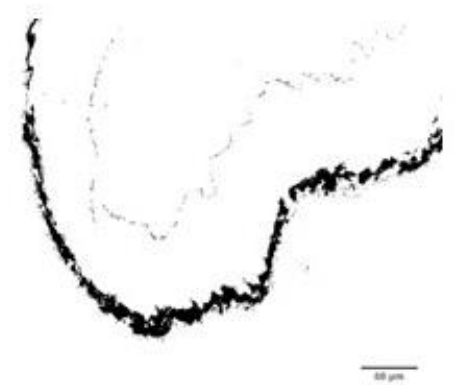

FIGURE 3: Masson's Trichrome Imaging with scale bar of $200 \mu \mathrm{m}$. A. Original image of an aorta. B: Selection of entire aorta. C: Selection of aorta without the nuclei. D. Selection of elastin in muscle layer. E. Selection of smooth muscle cells. F. Adventitia. G. Collagen in adventitia layer.

The same method of analysis used for the aortas was used for the hearts. The entire heart tissue excluding the nuclei was selected as described for the aortas. The amount of collagen (blue area) in the heat tissue was determined using the same technique described above in the aortas as well. 


\section{Histology- Immunofluorescent Staining:}

The immunofluorescence staining also started with rehydrating the tissue on the slides (one slide per heart or aorta as show in Table 7 below), with two five-minute rinses each in xylene, $100 \%$ ethanol, $95 \%$ ethanol, $90 \%$ ethanol, $85 \%$ ethanol, $80 \%$ ethanol, and $70 \%$ ethanol. All of the rinses were done in coplin jars. These rinses were followed by a five minute rinse in DI water and then a five minute rinse in phosphate buffered saline (PBS). The tissue was permeabilized with $1 \%$ triton in $1 \mathrm{M}$ glycine for 30 minutes at room temperature in a humidity chamber before being rinsed three times in PBS for five minutes each. Before the permeabilization step, an aquapen was used to draw a circle around the tissue slices to hold the triton mixture in place above the tissue. The 5\% BSA was added to the slide, which was placed in a humidity chamber. After three more rinses in PBS, the primary antibody solution was added with an overnight incubation at $4^{\circ} \mathrm{C}$ in the humidity chamber. The primary antibody solution was a 1:50 mixture of polyclonal rabbit anti-TGF- $\beta$ (sc-146, Santa Cruz Biotechnology) and PBS. After this step, everything was done in the dark since the secondary antibody and DAPI are photo-sensitive. Then the sample was rinsed 3 times in PBS, and the secondary antibody solution was added to incubate for 90 minutes at room temperature in the humidity chamber. The secondary antibody was Alexa Fluor 555 donkey anti-rabbit diluted a 1:15 solution with PBS. There were then another three rinses then five minutes of staining with $300 \mu \mathrm{M}$ DAPI diluted 1:10 in PBS to show nuclei. Finally samples were rinsed 3 times with PBS and three times with water before the 1 drop of FluorSave was added as mounting solution and covered with a coverslip.

Immunofluorescent Imaging

A confocal microscope, the Nikon Eclipse Ti, was used for the imaging (Table 7). The images were captured and analyzed using NIS Elements Analysis. The capture was done at 60x, 
and 3 images were taken per slice of aorta, making 6 images per slide since there were two slices per slide. The settings for all images captured was pinhole size at 1.0, frame rate was $1 / 8$, size at 1024, DAPI laser power at 1.25, TxRed laser power at 1.5, and light $\mathrm{HV}$ at 125 . When imaging the hearts, the TxRed laser power was 2.5 rather than the 1.5 used for the aortas because the 1.5 power did not show the fluorescence well in the hearts while 2.5 did.

TABLE 7: Number of mice in each group for Immunofluorescent staining

\begin{tabular}{|l|l|l|}
\hline Treatment & Number of hearts & Number of aortas \\
\hline NR Saline & 3 & 2 \\
\hline Rad Saline & 3 & 2 \\
\hline Chitocurc & 3 & 3 \\
\hline Lipids & 3 & 2 \\
\hline Alb/CCM & 2 & 2 \\
\hline CCM & 3 & 2 \\
\hline
\end{tabular}

Immunofluorescent Analysis

An image was opened in NIS Elements Analysis, the TD (white light) tab was chosen, and the white light was brightened as shown in Figure 4B. Then, the Draw Polygon tool from the Annotations and Measurements tab was used to separate the adventitia and smooth muscle of the aorta (in the case of imaging aortas) as shown in Figure 4C. Once this was done, the image was changed over to the TxRed tab, which contains the image of TGF- $\beta 1$ fluorescence. A 60 pixel by 120 pixel rectangle was created. Four of these rectangles were in the adventitia layer, 4 in the medial layer, and 4 not in the aorta measuring the background intensity. The mean of these 4 TxRed sum intensity measurements was taken for each section. For the ventricles, rather than four rectangles, a single 500 pixel x 500 pixel square was used for intensity measurements since the ventricles were not split into specific layers. To measure background noise, a 50 pixel x 50 
pixel square was placed in an area without tissue. The sum intensity of this measurement was used as the background signal and multiplied by 100 before subtracting out of all tissue measurements. The multiplication of the sum intensity from the background measurement by 100 was to have equivalent box sizes to the tissue measurement boxes for proper background subtraction.
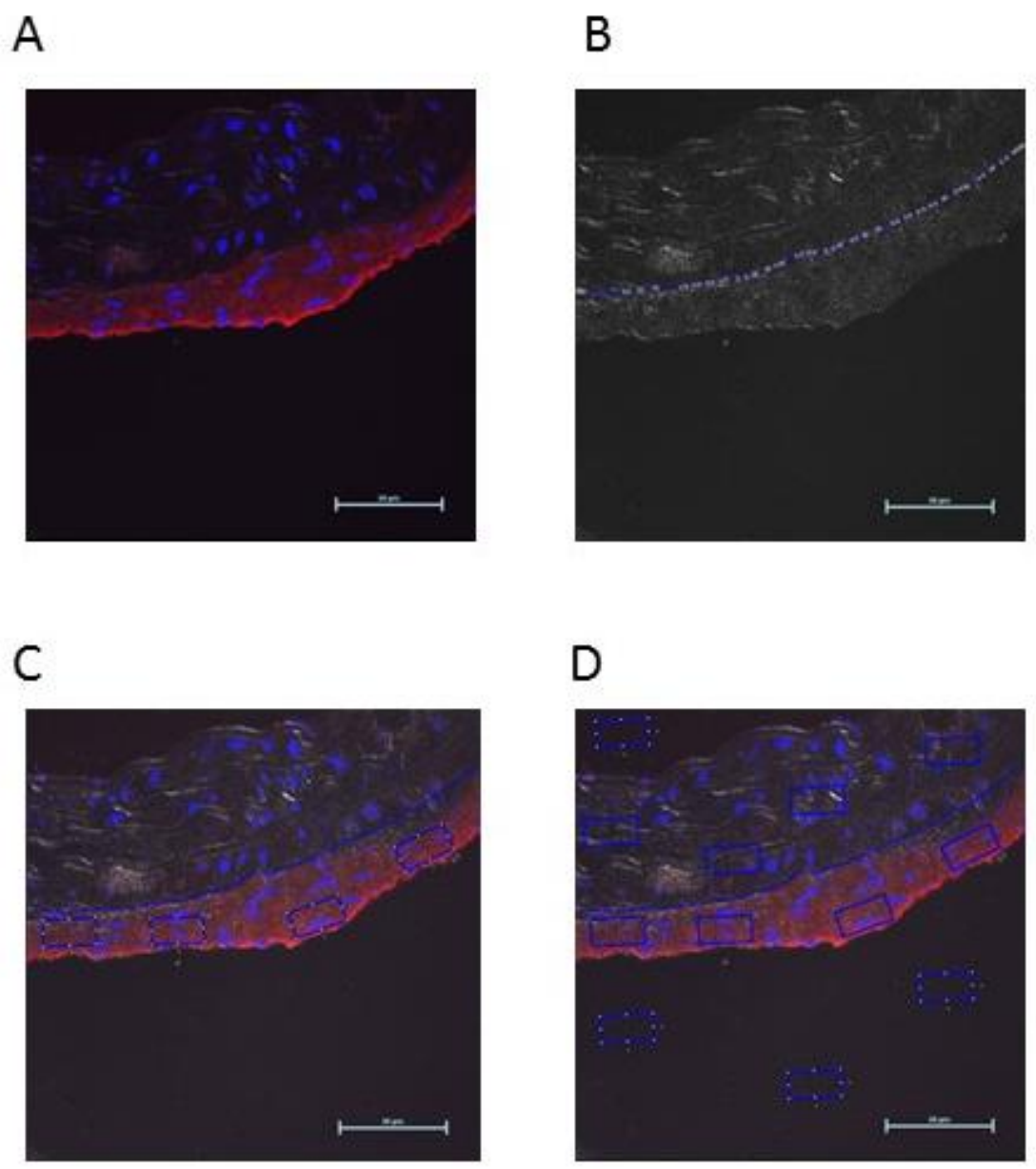

FIGURE 4: Quantification of TGF- $\beta 1$, shown in red, in immunofluorescent images with a scale bar of $50 \mu \mathrm{m}$. A: the stained aorta with all channels showing. B: the lightening of the white light image using the LUT panel, using the draw polygon tool of the annotations and measurements tab to divide the adventitia and smooth muscle layers. C. All channels shown and the calculation boxes in the adventitia. D. All layers and boxes shown with boxes in the background highlighted. 


\section{Statistics:}

The same statistical analysis techniques were used for the aortic outgrowth, ROS assays, and all histological tests. To fulfill aim 1, "Characterize the effect of ${ }^{56} \mathrm{Fe}$ on mouse cardiovascular tissue (heart and aorta)", the NR Sal and Rad Sal groups were compared using a $\mathrm{t}$ test with an alpha of 0.05 to indicate significance. To fulfill aim 2 "Examine the effectiveness of three independent curcumin drug delivery mechanisms at reducing mouse cardiovascular tissue damage caused by ${ }^{56} \mathrm{Fe}$, all groups that received radiation were compared using an ANOVA with an alpha value of 0.05 to indicate significance, after which Tukey tests were run for the post test.

The $\mathrm{n}$ values used for each assay are in the relevant methods sections, as are representative images for each treatment group. For each test, there is a graph in the relevant methods section containing the averages for each treatment group as well as the standard deviations.

\section{RESULTS AND DISCUSSION}

\section{Aortas:}

Aortic Outgrowth Assay:

Aortic outgrowth was measured by quantifying the area of the new cell growth from the sliced aorta using ImageJ. The cell outgrowth represents the aorta's ability to repair itself, which is hindered by radiation, as shown by compromised angiogenesis in a study by Soucy et al [40]. Ionizing radiation has also been shown to cause endothelial cell apoptosis[43], further hindering the growth of the aorta. The results of this study did not show a significant difference between Rad Sal and NR Sal groups, with a p value of 0.530 in the t-test. The treatment groups did not show a significant difference in outgrowth compared to Rad Sal either ( $p=0.160$ for the ANOVA). The resultant graph is shown below in Figure 5, and representative images for each 
treatment group are shown below in Figure 6. The aortic outgrowth trends in this study were replicated by a separate blinded analysis of the images. While there are no statistically significant differences between the groups, there are trends showing the Alb/CCM treatment inspired the highest outgrowth, with a 35\% increase in outgrowth area compared to Rad Sal. This increase in outgrowth could be from enhanced effectiveness of the antioxidant curcumin, which scavenges ROS that causes decreased aortic outgrowth. However in other studies curcumin has been shown to decrease angiogenesis. The effects of curcumin on cell proliferation which is important for angiogenesis is variable throughout literature. Curcumin's effect on angiogenesis is possibly dependent on dosage and tissue. Curcumin has been found to hasten wound healing, increasing proliferation and collagen synthesis at a wound site $[44,45]$. In a rat experiment with topically applied curcumin for wound healing, experimental rats received $200 \mu 1$ of the curcumin at a concentration of 40-mg/kg-body weight, and in that experiment curcumin led to proliferation [44]. In other applications, such as cancer prevention, curcumin has been shown to limit proliferation and angiogenesis [46]. In the Arbiser et. al study, concentrations of curcumin of 0.5- $10 \mu \mathrm{M}$ were added to primary endothelial cells in the eye, with curcumin inhibiting endothelial growth, the largest cell decrease happening at $10 \mu \mathrm{M}$. Given the different doses of curcumin released for each nanoparticle in our study (listed in Table 2), decreased aortic outgrowth could be from decreased proliferation induced by curcumin in the Lipids, Chitocurc, and CCM treatments.

Compared to the study by Soucy et al. [40], the results described here show less effect of radiation Soucy et al used gamma radiation at doses of $0.5 \mathrm{~Gy}$ and above rather than $20 \mathrm{cGy}$ of ${ }^{56} \mathrm{Fe}$ radiation. In the Soucy et al. study, the higher doses of radiation reduced aortic outgrowth, and the lowest does of 0.5 Gy dose had a small significant effect (reduction of outgrowth from 
$1.00 \pm 0.22$ at $0 \mathrm{~Gy}$ to $0.897 \pm 0.022$ at $0.5 \mathrm{~Gy}, \mathrm{p}<0.05)$. Whereas in this study, the radiation dose did not show a significant effect, with a slight increase in outgrowth due to radiation. This unexpected result could be due to the lower dose of radiation and different type of radiation exposure used here. Since the variation in the data was higher in this study, this could be another reason why the radiation effects did not prove to be significant as well as explaining why there was a reverse of the expected trend in the Rad Sal and NR Sal groups.

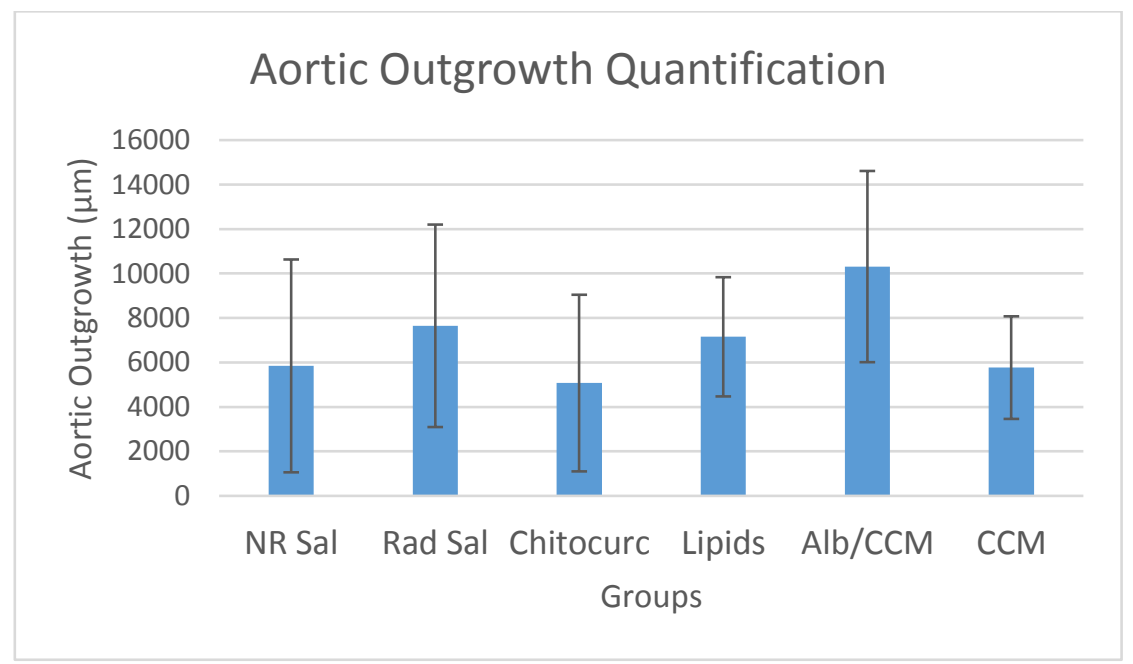

FIGURE 5: Aortic Outgrowth Quantification graph - averages of outgrowth area with standard deviations. 
A

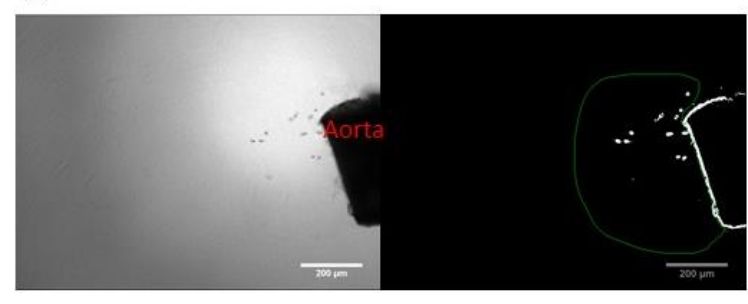

C

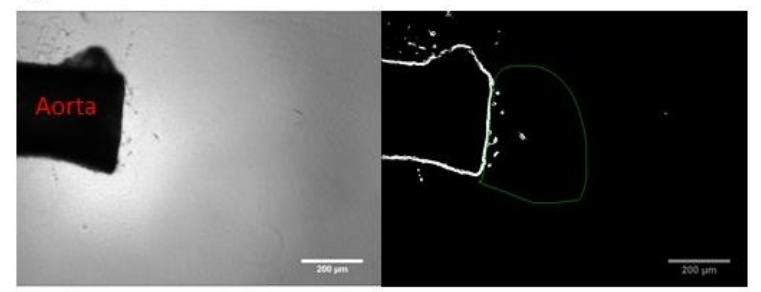

$\mathrm{E}$

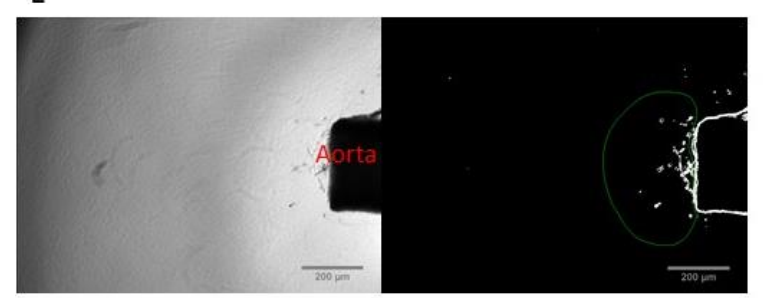

FIGURE 6: Representative images for Aortic Outgrowth Assay - A: NR Sal, B: Rad Sal. C: Chitocurc. D: Lipids. E: Alb/CCM. F: CCM

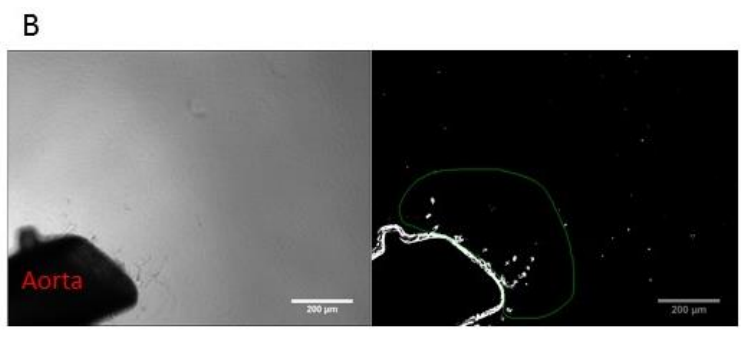

D

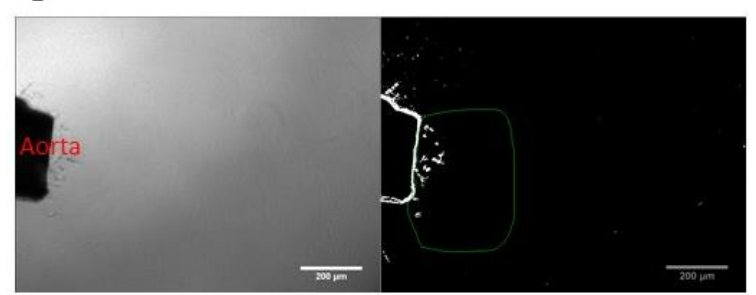

$\mathrm{F}$

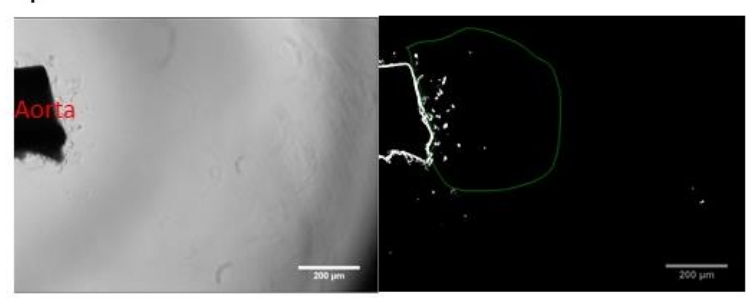

\section{Chitocurc. D: Lipids. E: Alb/CCM. F: CCM}

\section{Immunostaining:}

Immunostaining was performed on several of the aortic outgrowth assay wells with higher levels of outgrowth to ensure that the cells growing from the cut ends of the aorta in the aortic outgrowth assay were in fact endothelial cells. It has been observed in a study by Soucy et al. that in aortic outgrowth, the majority of the outgrowth comes from the endothelium [40]. Endothelial cells are the ones that demonstrate the growth and repair of the vasculature. In Figure 7 below, the white light demonstrates the full image of the outgrowth, the blue image shows the DAPI stained nuclei, and green images show the IB4 stained endothelial cells. Although the staining is very dim, the majority of outgrowth was stained lightly as endothelial cells, rather than cells from the connective tissue. 


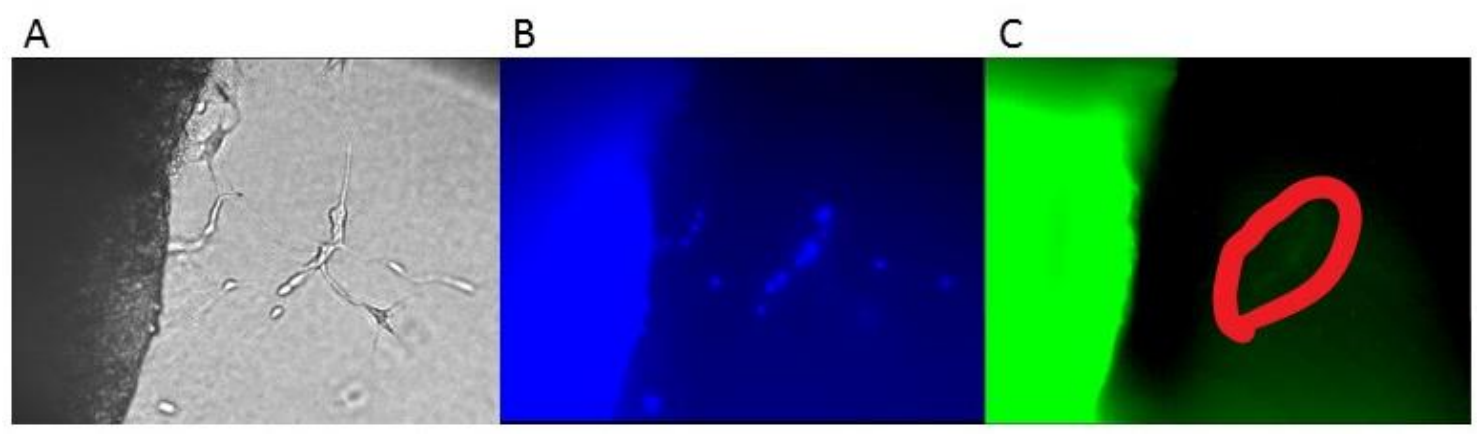

FIGURE 7: IB4 immunostained aorta - A: Brightfield image. B: violet filter/DAPI image. C: blue filter/FITC image. Due to low intensity in the IB4 stained image (image C), additional post processing was done to enhance visualization. Background subtraction was done in ImageJ using the max greyscale value found in the area in the bottom right corner. Then an unsharp mask was applied with a radius (sigma) of 80 pixels and mask weight (0.1-0.9) of 0.65 .

\section{ROS Assay:}

The ROS assay was performed to assess the amount of ROS in the aortas. These measurements examined the ROS levels in aortas 14 days after radiation. The reason for examining the amount of chronic ROS was because the treatments contained curcumin, an antioxidant, which can reduce ROS levels. The results are shown in Figure 8 below. When the results were run in Minitab, there were no significant differences shown between the NR Sal and Rad Sal treatment groups, with a p value of 0.772 . This data suggests the radiation did not increase chronic ROS in aortas. The ANOVA run for the treatment groups excluding NR Sal had a $\mathrm{p}$ value of 0.719 , showing a lack of effect from the CCM treatments. All of the groups except Chitocurc had averages very similar to each other, with ROS in mouse tissue treated with Chitocurc demonstrating a 29\% decrease in ROS compared to Rad Sal. One possible explanation for the reduced ROS in Chitocurc treated tissues was that Chitocurc released CCM over the course of 19 days in this treatment, so the antioxidant release could still be occurring at the time of. These results differ from a separate ROS assay done on the bone marrow of the same mice by a collaborator in which there was no significant difference between the NR Sal and Rad Sal, but all the drug treatments did lower the ROS levels. The differences in results might exist due to the 
fact that the collaborator used fresh tissue in their study while this study used frozen tissue to assess the ROS in the aortas. In addition, the sensitivity to radiation and ROS may vary from tissue to tissue. However, both assays of 2 different tissues found that ROS levels were not elevated 14 days after the radiation treatment in saline treated mice.

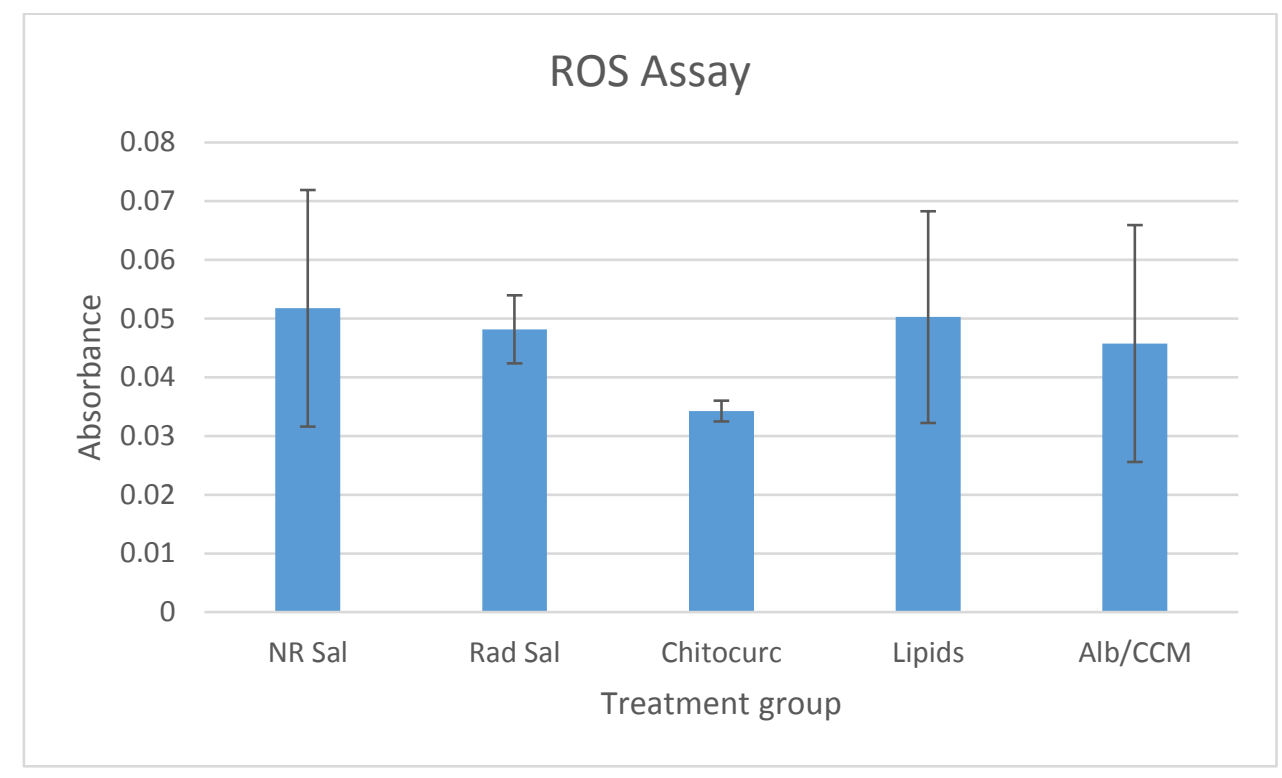

FIGURE 8: Graph of the ROS assay.

Histology:

$\underline{\text { Histology }-\mathrm{H} \& \mathrm{E}}$

In order to see if the radiation and treatments effected the heart and aorta tissue organization, hematoxylin and eosin staining was performed. Hematoxylin and Eosin staining is a global stain of nuclei in which stains nuclei blue and smooth muscle red. The amount of smooth muscle per nuclei was calculated using ImageJ to examine changes in cell growth. The calculations were done measuring the amount of smooth muscle per nuclei with the assumption that a smaller area of smooth muscle per nuclei would indicate cell multiplication. The representative images are in Figure 9 below, and the graph of the average amount of smooth muscle area per nuclei is in Figure 10 below. There was not a statistical difference between the 
NR Sal and Rad Sal groups, with a t test $p$ value of 0.760 . There was also no difference between any of the drug treatment groups and the Rad Sal group, with a p value 0.454 . The trends showed Rad Sal only had a $6 \%$ decrease in smooth muscle per nuclei compared to NR Sal, while the CCM group had a $21 \%$ increase in smooth muscle per nuclei compared to Rad Sal, suggesting ventricular smooth muscle cell hypertrophy. Chitocurc was very close in value to NR Sal with an $8 \%$ increase in smooth muscle per nuclei compared to Rad Sal, while Lipids actually showed a $12 \%$ decrease in smooth muscle per nuclei compared to Rad Sal, suggesting an increase in ventricular smooth muscle cell proliferation.
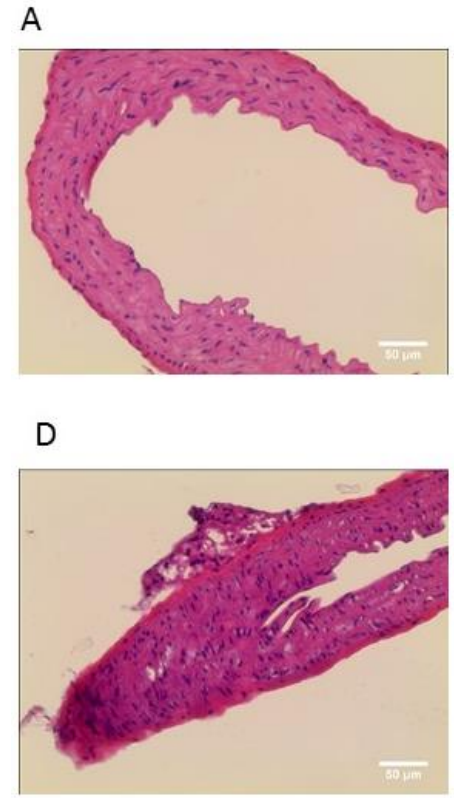

B

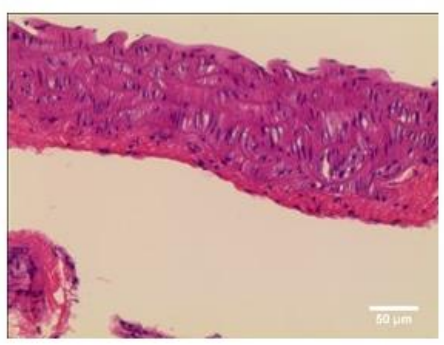

$\mathrm{E}$

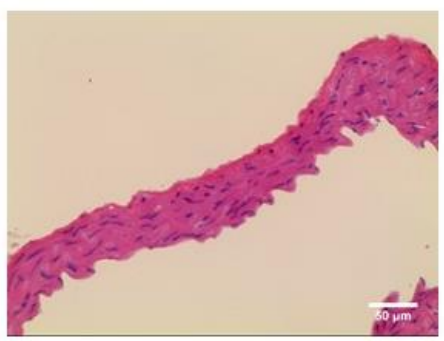

C

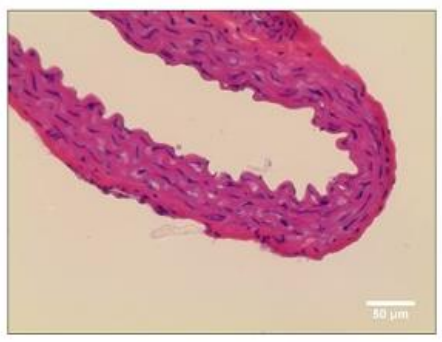

F

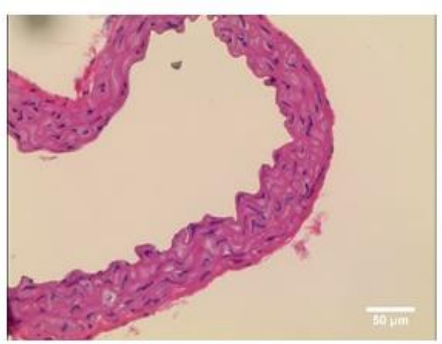

FIGURE 9: Representative Images for the amount of smooth muscle per nuclei in the aortas. A: NR Sal, B: Rad Sal. C: Chitocurc. D: Lipids. E: Alb/CCM. F: CCM 


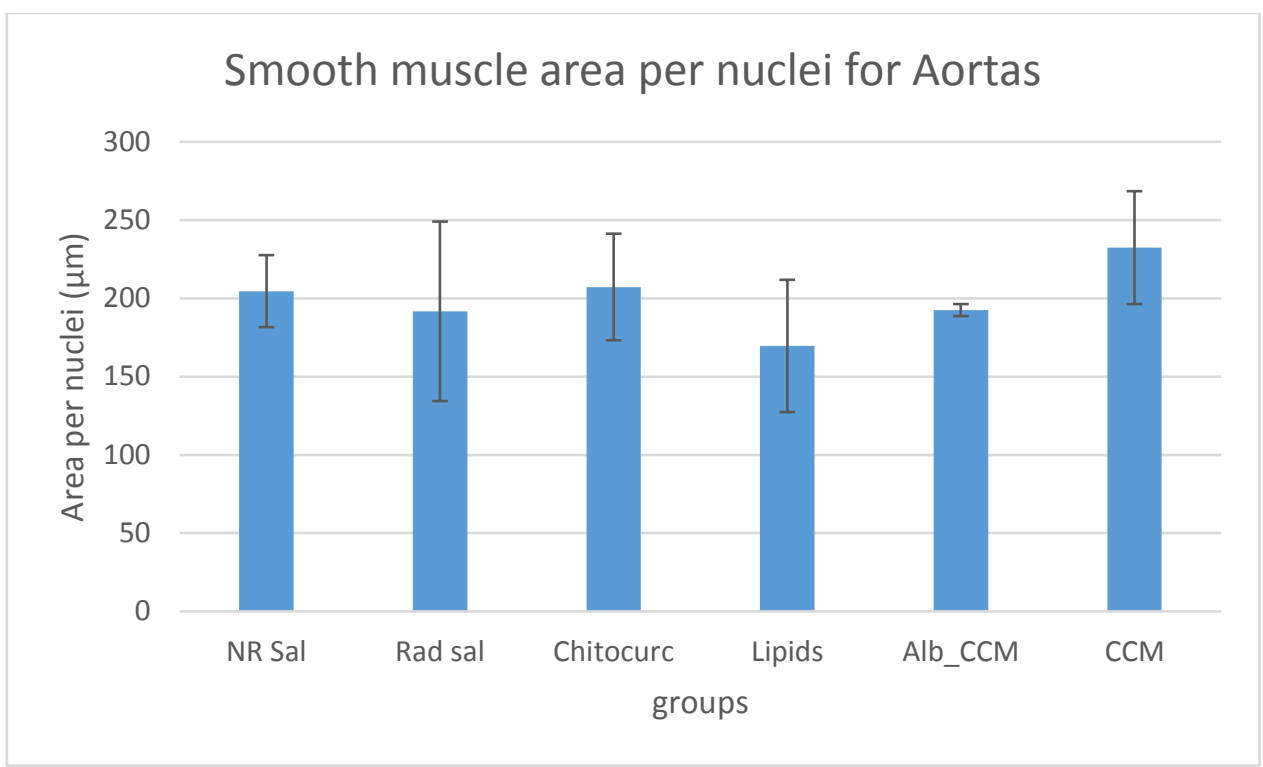

FIGURE 10: Graph of the averages and standard deviations for the smooth muscle per nuclei measured in $\mu \mathrm{m}^{2}$ in the $\mathrm{H} \& \mathrm{E}$ stained aortas.

Histology - Wall Thickness:

The wall thicknesses of the aortas were compared between treatment groups to test if the radiation caused thickening of the aorta walls. Thickening of the aorta walls would lead to decreased flexibility of the vasculature and so increased blood pressure and cardiovascular damage. Thickening of other arteries such as the carotid arteries has been shown as a result of ${ }^{56} \mathrm{Fe}$ radiation, shown in the thickening of the intima as show by immunohistochemical and immunofluorescence staining at 13 weeks after radiation[47]. Similar thickening was expected of the aorta. The representative images are shown below in Figure 11 while the graph of the averages and standard deviations for each group is in Figure 12. There were no significant differences between NR Sal and Rad Sal, with a p value of 0.240 in the $t$ test, but the Rad Sal group had a 39\% increase in wall thickness compared to the NR Sal group. The different drug treatment groups did not have significant differences either, with a p value of 0.501 in the ANOVA. The Alb/CCM and Lipids treatments lead to thinner aortas than the Rad Sal group with a $38 \%$ and $26 \%$ decrease in wall thickness compared to Rad Sal respectively. This data shows 
some protective ability from vessel thickening from the curcumin delivered by Alb/CCM and Lipids. There are also lower standard deviations for the NR Sal, Chitocurc, and Alb/CCM groups, with standard deviations of $0.35(0.6 \%), 5.4(7.1 \%)$, and $4.9(9.7 \%)$ respectively compared to the standard deviation of 24 (29\%) for the Rad Sal group. This could imply that the radiation effects were highly varied between mice and be a consequence of a low $n$ value.

A

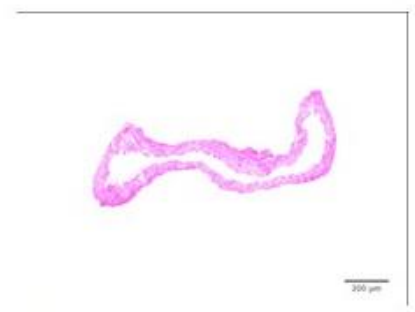

D

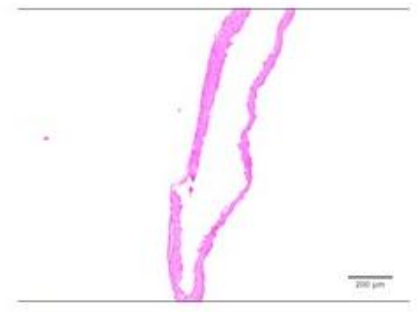

B

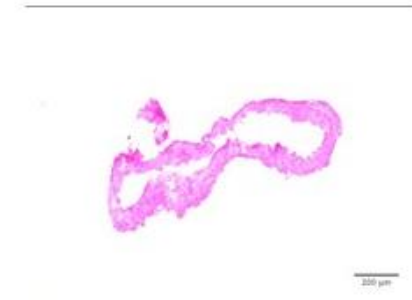

$E$

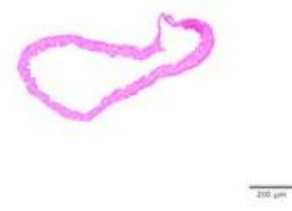

C

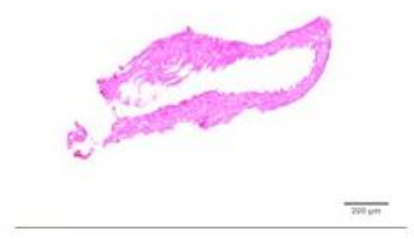

$\mathrm{F}$

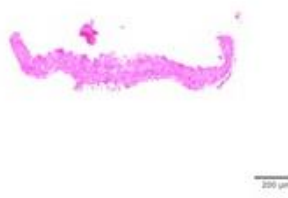

FIGURE 11: Representative images for the wall thickness of the aorta. A: NR Sal, B: Rad Sal. C: Chitocurc. D: Lipids. E: Alb/CCM. F: CCM 


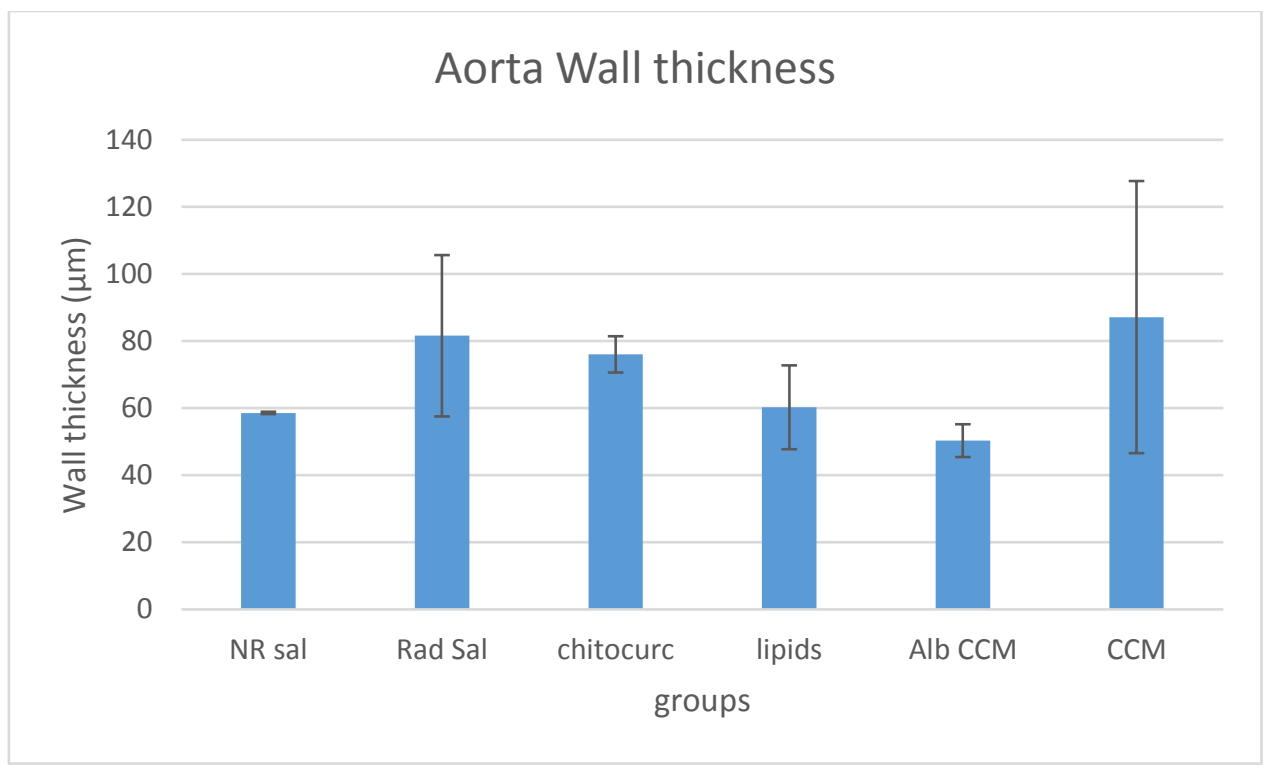

FIGURE 12: Graph of the wall thicknesses of the H\&E stained aortas.

Histology - Masson's Trichrome:

Masson's Trichrome is a three color stain that stains muscle tissue dark pink/red, ECM light blue, and nuclei dark blue. This stain allows the separate layers of the aorta to be differentiated into the media layer and adventitia layer. The thicknesses of the adventitia layer of the Masson's trichrome stained aortas was measured to determine whether a certain layer was thickening rather than the entire aorta. For the adventitia thickness alone, there was no significant difference between NR Sal and Rad Sal as a result of radiation with a p value of 0.498 . Nor was there a difference between Rad Sal and the treatment groups, with a p value of 0.788 . The adventitia layer thickness was also measured as a percentage of the total aorta thickness. There was no significant difference in the \% of the total aorta thickness that was adventitia between the NR Sal and Rad Sal groups, with a p value of 0.736 . Nor was there a difference in the percentage of the aorta that was adventitia as a result of the treatments, with a p value of 0.819 between the treatment groups and Rad Sal. Below in Figure 13 the thicknesses of each layer of the aorta are shown for each group, and the \% of the total thickness that is adventitia is shown in Figure 14. 
All treatment groups had higher percentages of adventitia compared to NR Sal. The representative images are shown in Figure 15 below, with the strip of blue on each aorta being the adventitia layer.

After finding the amount of adventitia, the amount of the adventitia that is the ECM protein collagen was measured. In this case, it was area rather than thickness that was found in ImageJ. The collagen is the specific ECM protein that is linked to fibrosis, so it serves as a signifier of cardiovascular damage. The radiation did not show a significant effect on the percent collagen within the aorta compared to NR Saline, with a p value of 0.777 in the $t$ tests, none of the treatments were significantly different than the Rad Saline with a p value of 0.840 in the ANOVA. There were very high standard deviations as shown in Figure 16, with standard deviations as high as $92 \%$ of the $\%$ collagen. This standard deviation was partially due to differences between mice and partially due to the method of analysis which required identifying collagen from the rest of the adventitia by eye. The Rad Sal group has a higher average level of collagen than the NR Sal group, with a $23 \%$ increase in collagen area, though the standard deviations are high enough that there is no significant difference. 


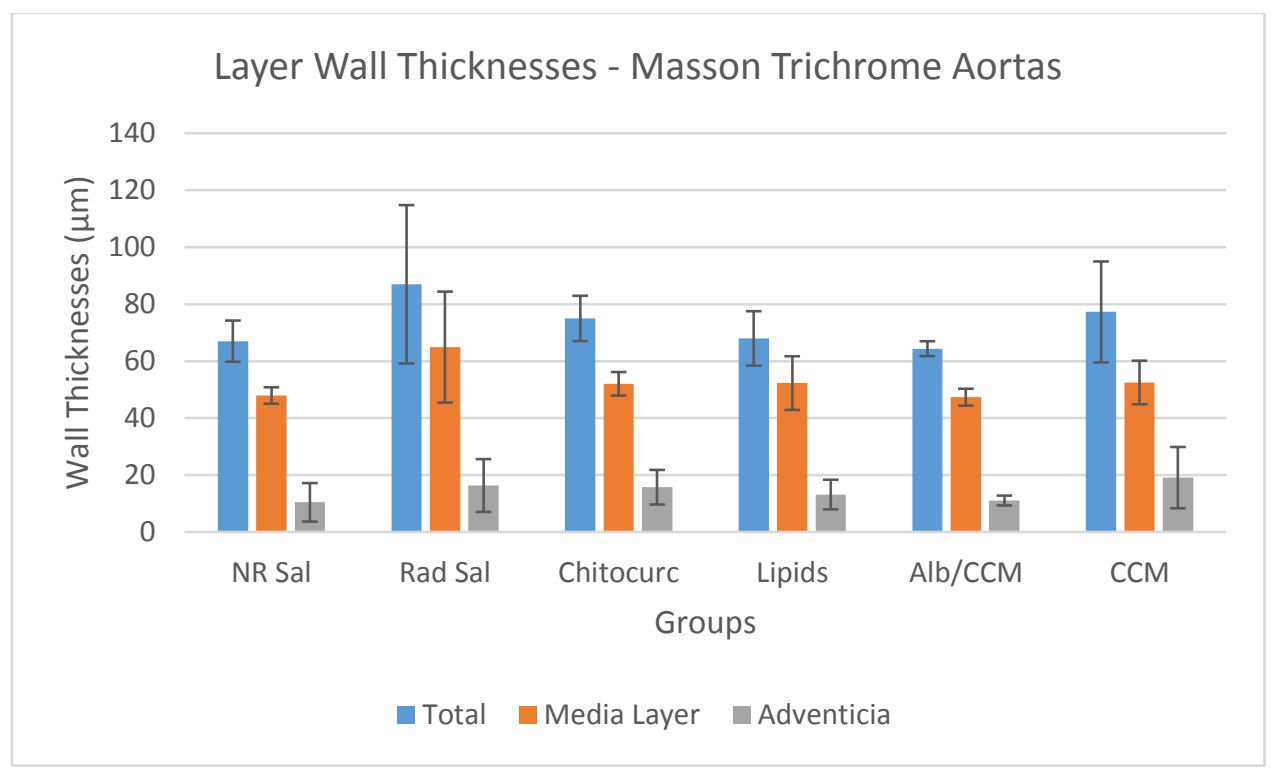

FIGURE 13: Graph of the wall thicknesses of each layer of the aorta stained with Masson's trichrome.

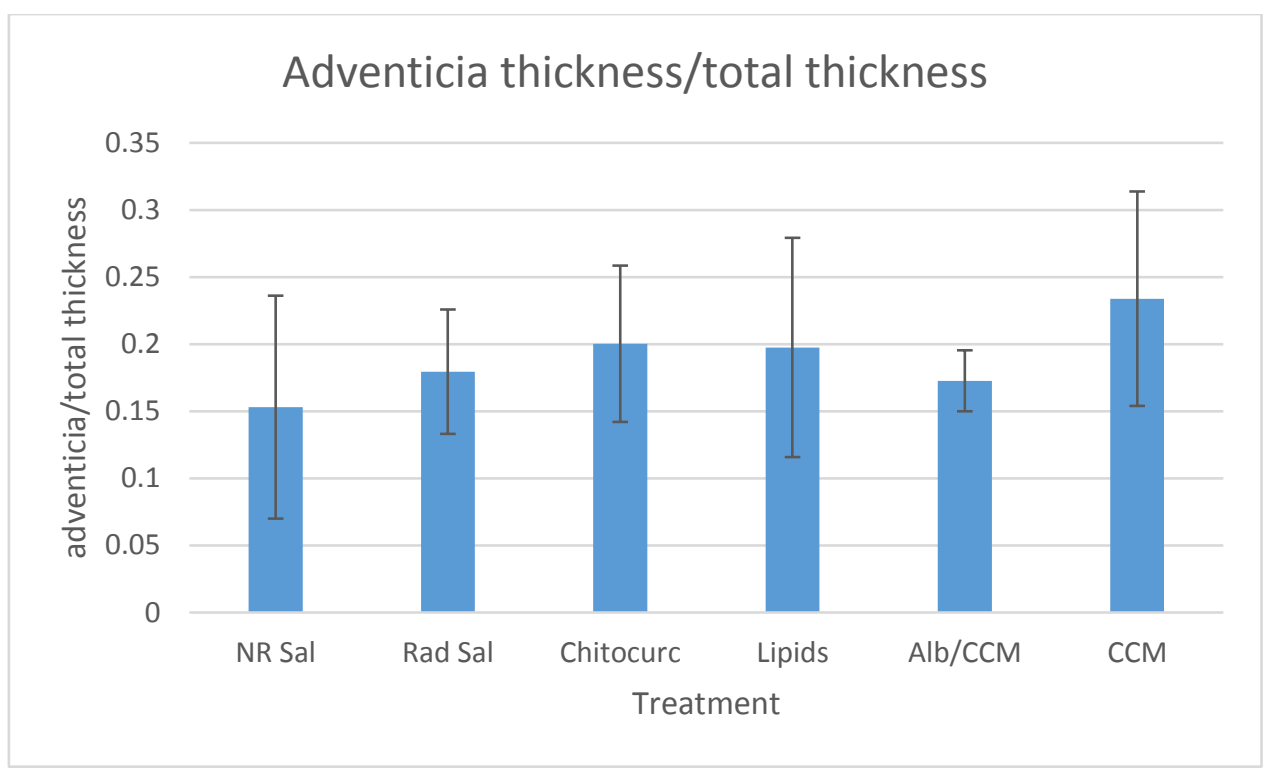

FIGURE 14: Graph of the adventitia thickness divided by the total aorta thickness for each group. 
A

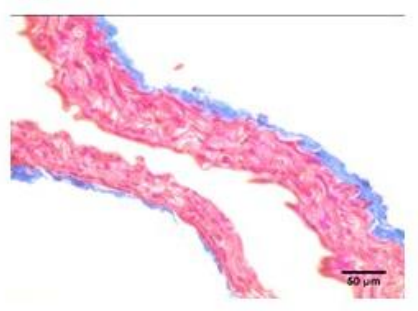

$\mathrm{D}$

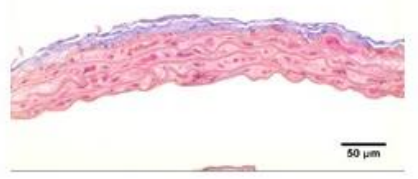

B

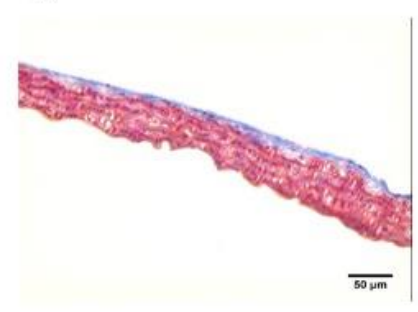

$\mathrm{E}$

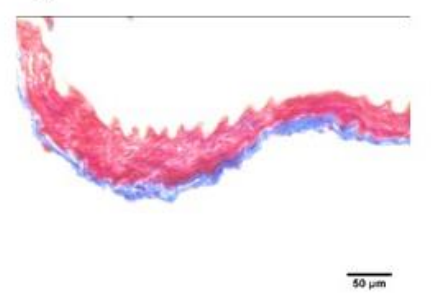

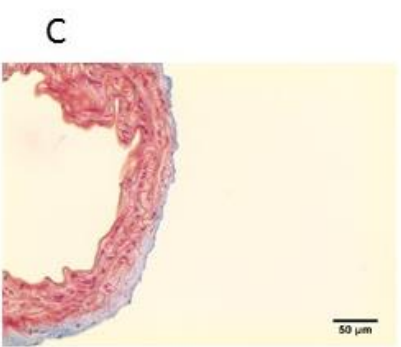

$\mathrm{F}$

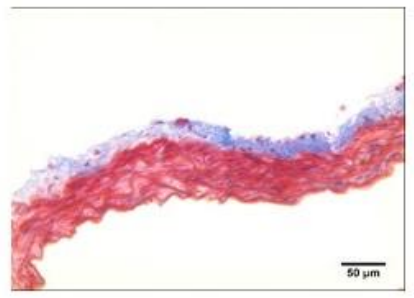

FIGURE 15: Representative images of wall thicknesses of aortas stained with Masson's trichrome. A: NR Sal, B: Rad Sal. C: Chitocurc. D: Lipids. E: Alb/CCM. F: CCM

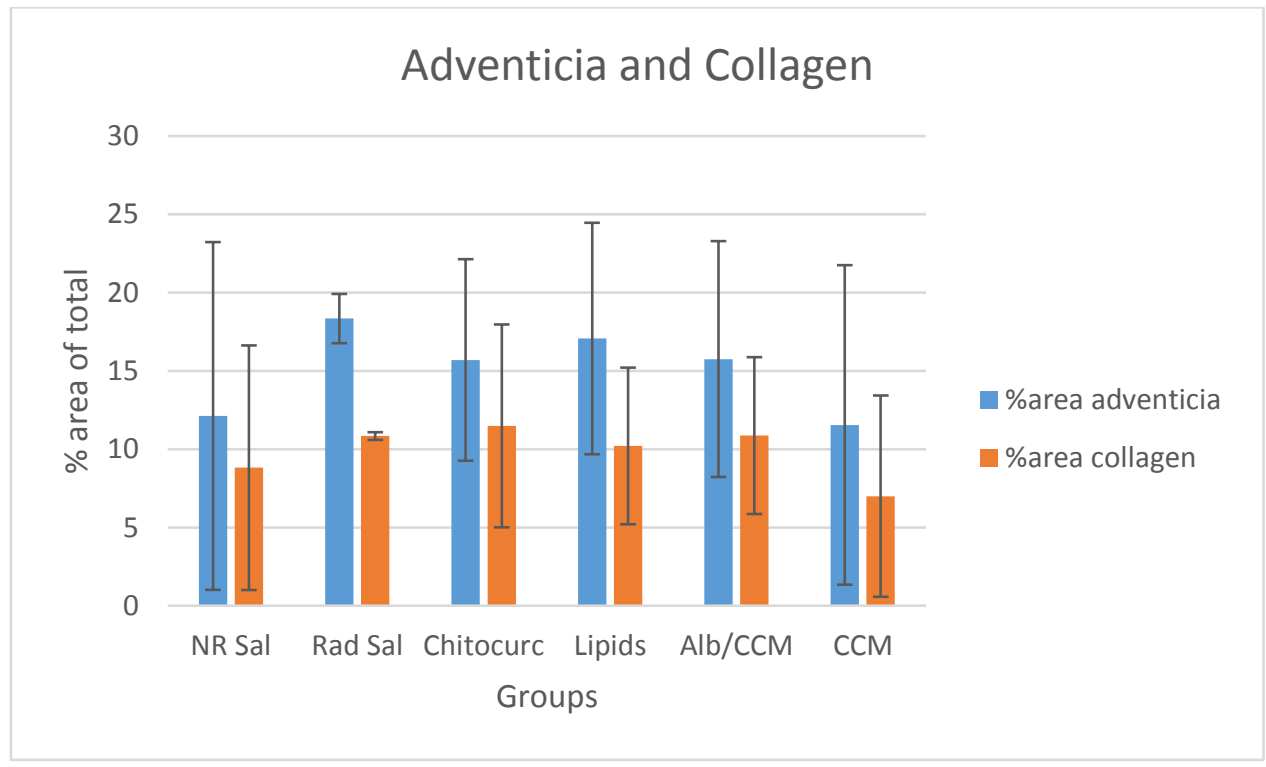

FIGURE 16: Graph of the \% of the total aorta area that is adventitia and the amount of the total aorta area that is collagen.

Histology - Immunostaining:

TGF- $\beta 1$ is a signaling molecule highly expressed in fibrosis. The images for the aortas in which TGF- $\beta 1$ is stained for are shown below in Figure 17, and the averages of the intensity of the fluorescence of the TGF- $\beta 1$ and their standard deviations are in Figure 18. The standard 
deviations are very high between groups, resulting data difficult to draw conclusions from. There is no significant effect of radiation according to the $t$ test between NR Sal and Rad Sal with a p value of 0.600 . There was also no significant effect of the drug treatments according to the ANOVA with the $\mathrm{p}$ value of 0.832 . There are trends indicating that the NR Sal and Lipids groups have the lowest amount of TGF- $\beta 1$ compared to Chitocurc or Rad Sal, however, the standard deviation is too high to be certain. The standard deviations are lower in the case or NR Sal and Lipids compared to those of the Rad Sal and Chitocurc treatments, indicating that the increase in TGF- $\beta 1$ expression is variable between groups and within groups.

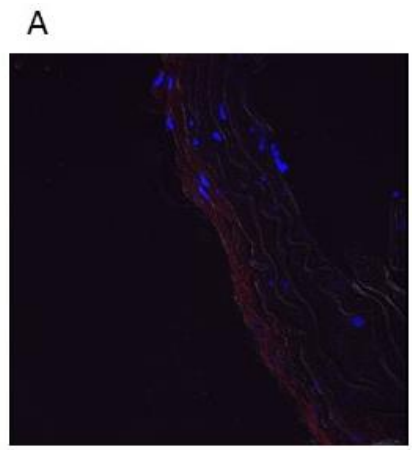

D

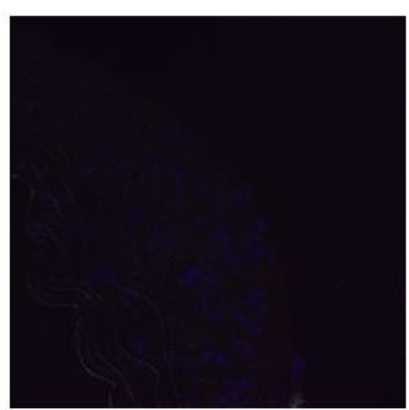

B

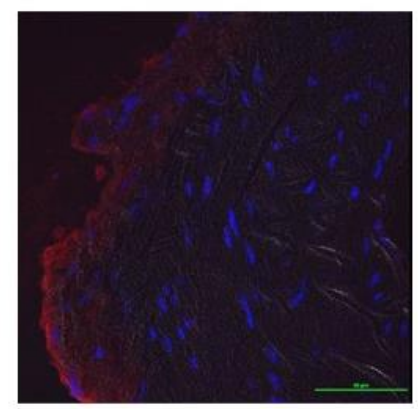

$\mathrm{E}$

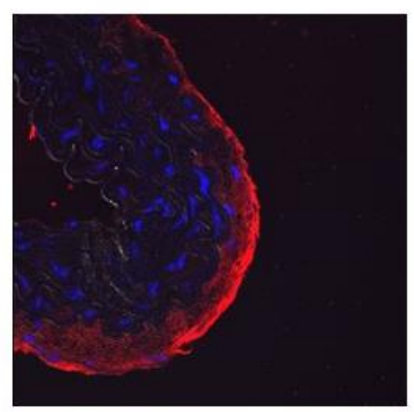

C

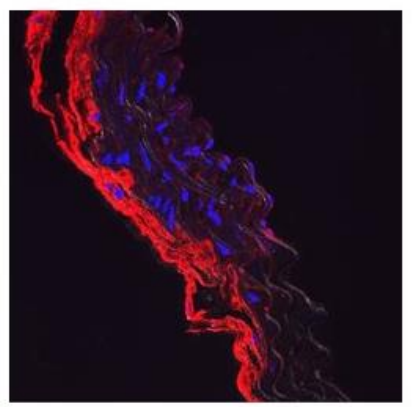

$\mathrm{F}$

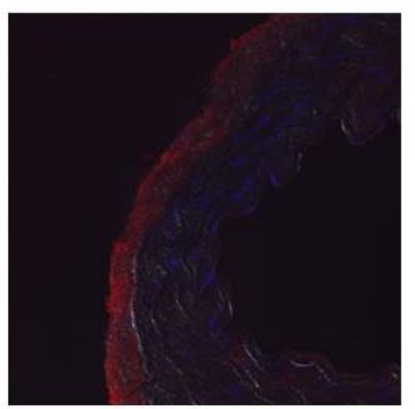

FIGURE 17: Representative images for the immunostained histology aorta slides. A: NR Sal, B: Rad Sal. C: Chitocurc. D: Lipids. E: Alb/CCM. F: CCM 


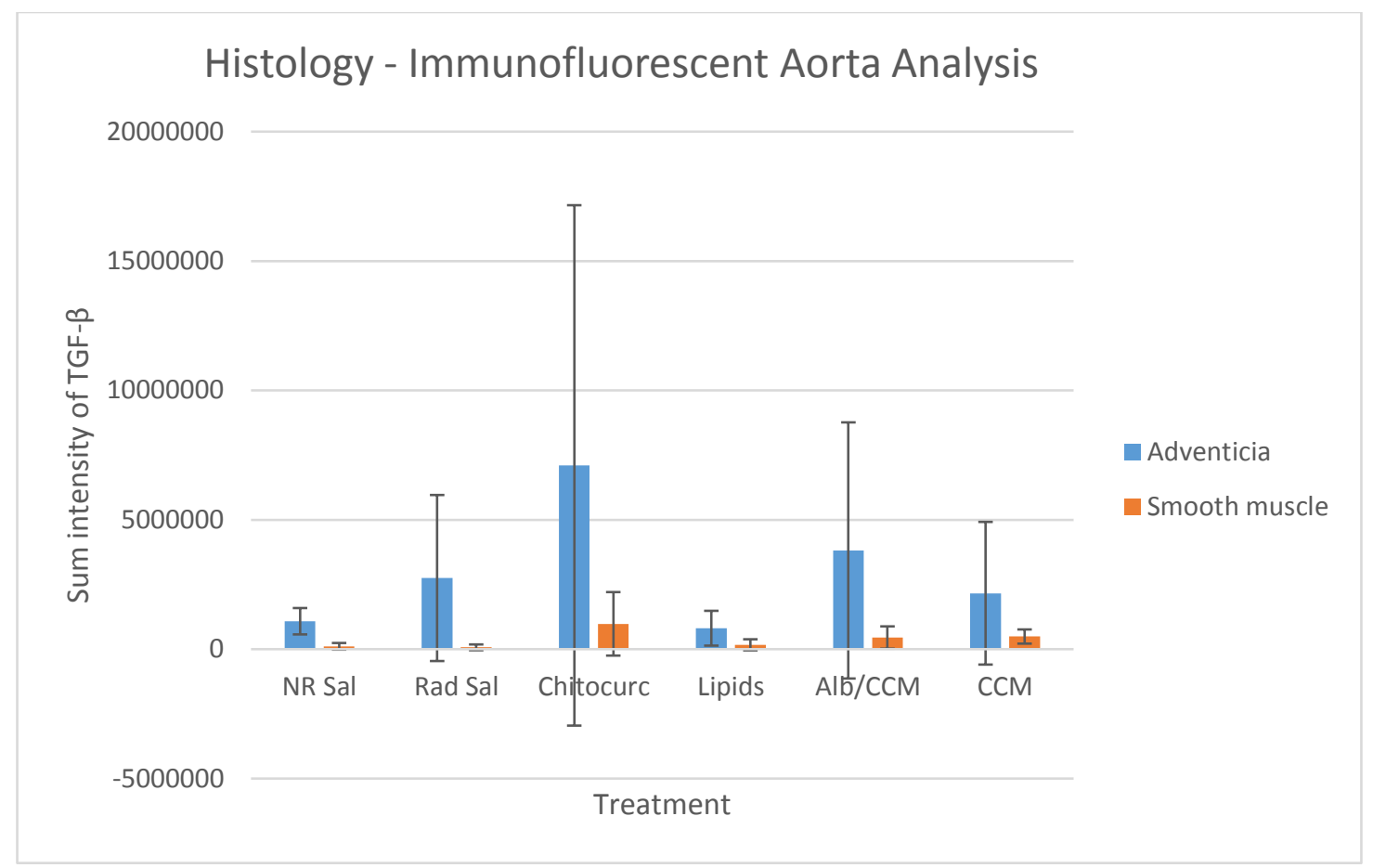

FIGURE 18: Averages and standard deviations for immunostained aortas for both the adventitia and smooth muscle.

\section{Ventricles:}

Histology:

Histology $-\mathrm{H} \& \mathrm{E}$

In order to see if the radiation and treatments effected the heart, hematoxylin and eosin staining was performed. Hematoxylin and Eosin staining is a global stain of nuclei in which stains nuclei blue and cardiac muscle red. The representative images are in Figure 19 and 20 for the right and left ventricles respectively, and the graph of the average amount of cardiac muscle area per nuclei is in Figure 21 below. The radiation had a significant effect on the right ventricle area per nuclei, with a $\mathrm{p}$ value of 0.041 in the $\mathrm{t}$ test. The drug treatments did not have a significant impact on area per nuclei, with a $\mathrm{p}$ value of 0.997 from the ANOVA. For the right ventricle, the radiation caused a $16 \%$ decrease in cardiac muscle area per nuclei, comparing Rad Sal to NR Sal. None of the antioxidant treatments altered this effect, with the largest change being in Chitocurc with a $3.6 \%$ increase in cardiac muscle area per nuclei compared to Rad Sal. 
There was no significant effect of radiation in the amount of cardiac muscle per nuclei in the left ventricle, with a $p$ value of 0.562 in the $t$ test. There was not a significant effect of the drugs either, with a $\mathrm{p}$ value of 0.347 in the ANOVA. There was only a $6 \%$ decrease in cardiac muscle area per nuclei, and the only group that increased the cardiac muscle area per nuclei was CCM, with an increase of $13 \%$ compared to Rad Sal.
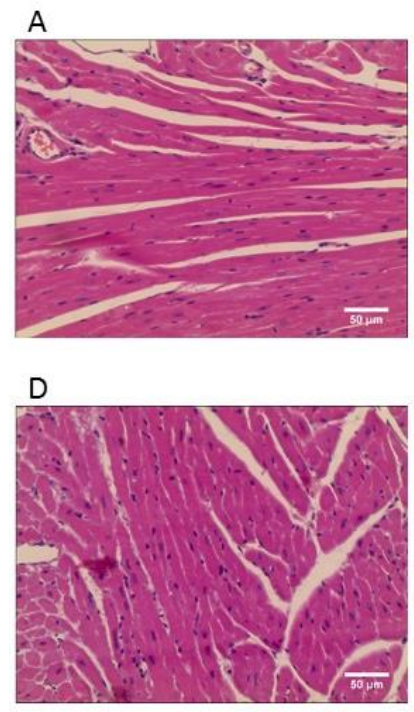

B

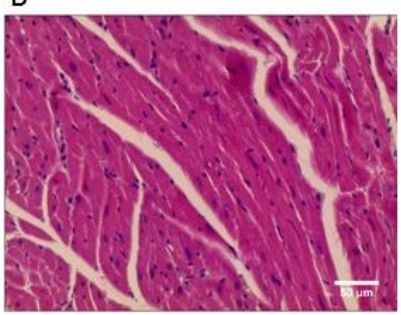

$\mathrm{E}$

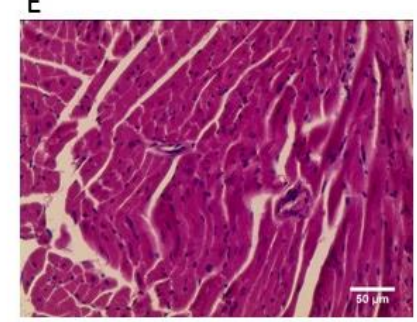

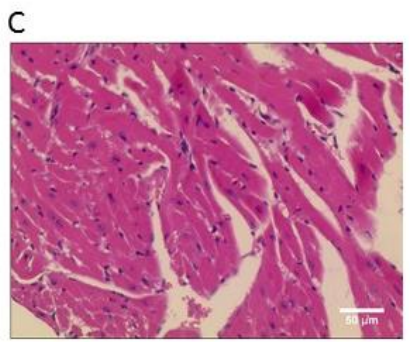

$\mathrm{F}$

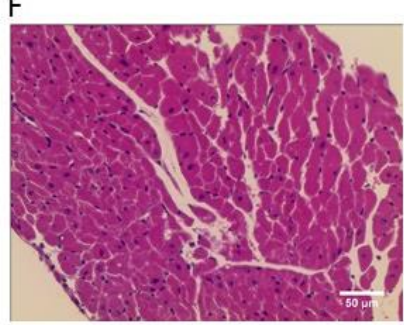

FIGURE 19: Representative Images for the amount of cardiac muscle per nuclei in the right ventricle. For each image, the original, smooth muscle, and nuclei are shown. A: NR Sal, B: Rad Sal. C: Chitocurc. D: Lipids. E: Alb/CCM. F: CCM 


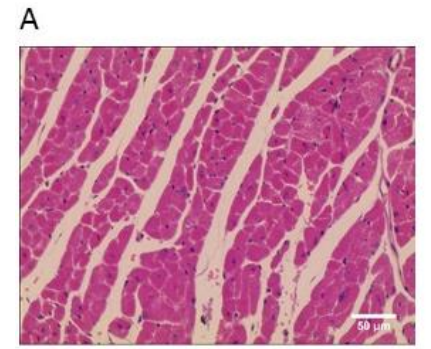

D

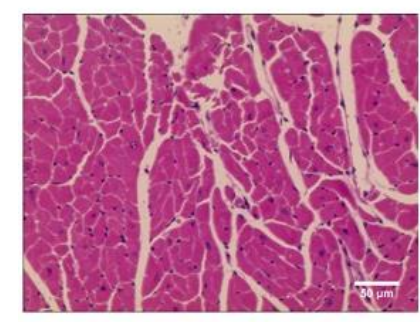

B

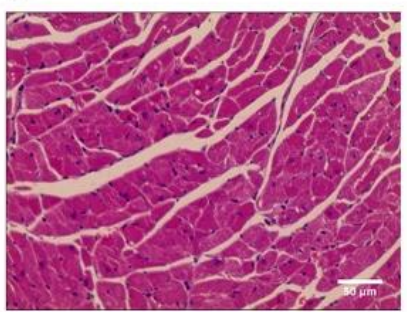

$E$

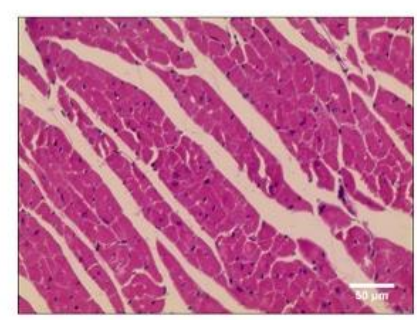

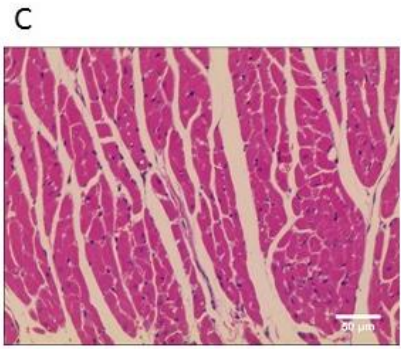

$\mathrm{F}$

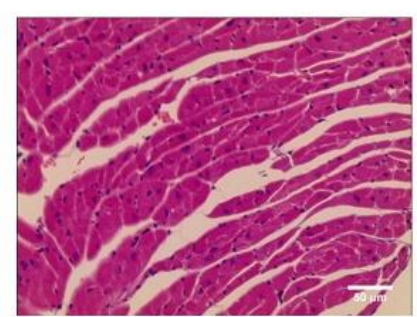

FIGURE 20: Representative Images for the amount of cardiac muscle per nuclei in the left ventricle. For each image, the original, smooth muscle, and nuclei are shown. A: NR Sal, B: Rad Sal. C: Chitocurc. D: Lipids. E: Alb/CCM. F: CCM

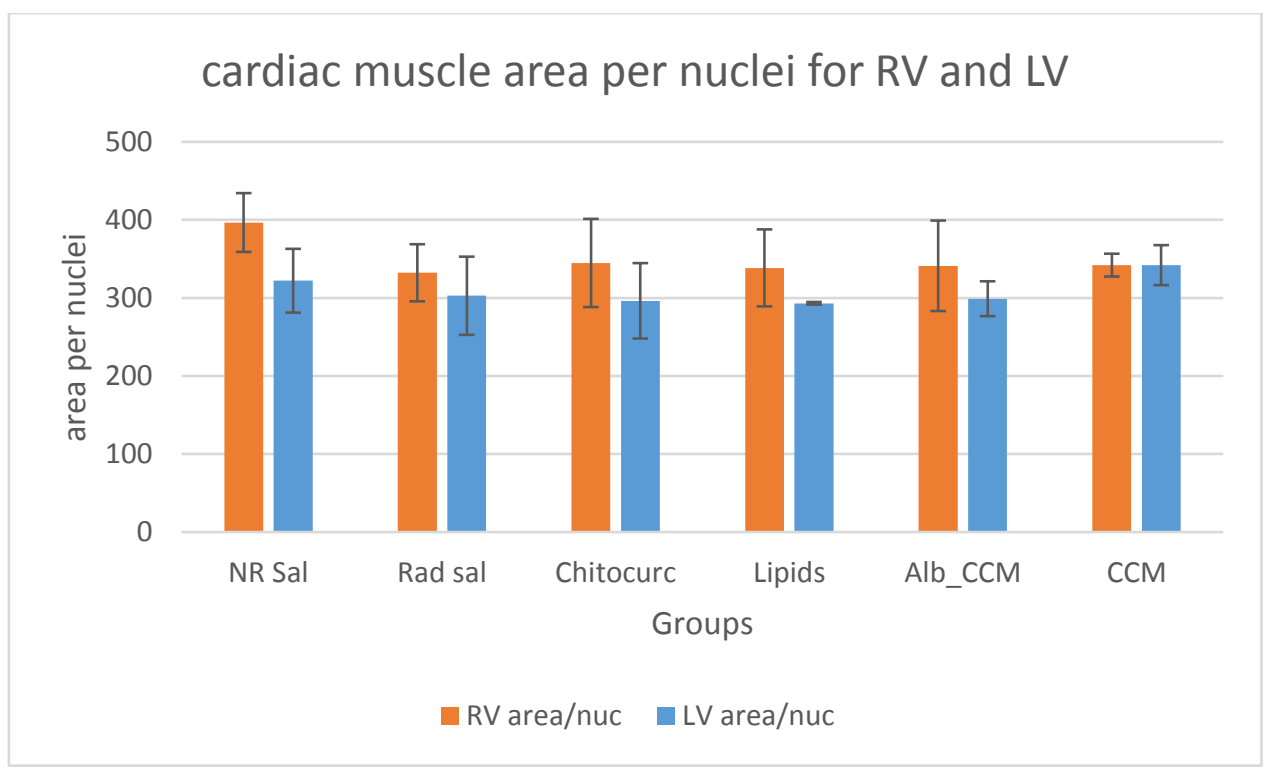

FIGURE 21: Graphs of the averages and standard deviations for the cardiac muscle in $\mu \mathrm{m}$ per nuclei in the $H \& E$ stained right and left ventricles

$\underline{\text { Histology - Wall Thickness }}$

The wall thicknesses of the ventricles were compared between treatment groups to test if the radiation caused thickening of the ventricle walls. Effects of thickening of the ventricle walls 
could lead to similar effects as the stiffening of arteries, with decreased flexibility and increased blood pressure. This would imply cardiac hypertrophy which indicates increased effort for the heart to pump. The representative images of the right and left ventricle are shown below in Figure 22 and 23, while the graph of the averages and standard deviations for each group is in Figure 24.

For the right ventricle, there is a significant difference between the NR Sal and Rad Sal showing an effect of radiation, with a p value of 0.026 in the $\mathrm{t}$ test. The Rad Sal shows a $15 \%$ increase in wall thickness compared to the NR Sal. There is not a significant effect of the antioxidant treatments in comparison to Rad Sal, with a p value of 0.497. All antioxidant treatments showed a trend of a slight decrease in wall thickness, with Chitocurc, Lipids, $\mathrm{Alb} / \mathrm{CCM}$, and CCM showing a 14\%, 13\%, $8 \%$, and $12 \%$ decrease respectively compared to Rad Sal.

For the left ventricle, there is not a significant difference between NR Sal and Rad Sal, with a $\mathrm{p}$ value of 0.343 , but there is an effect of the antioxidant treatments since the ANOVA has a $p$ value $<0.001$. In this case, rather than a thickening as a result of radiation, there seems to be a thinning, with Rad Sal decreasing in wall thickness by $22 \%$ compared to NR Sal. The $\mathrm{Alb} / \mathrm{CCM}$ and CCM antioxidant treatments seem to prevent this ventricular thinning, with an increase of $55 \%$ and $61 \%$ increase in wall thickness respectively compared to Rad Sal. The Tukey test used as a post test for the ANOVA resulted in an adjusted $p$ value of 0.018 between Alb/CCM and Rad Sal. There was an adjusted p value of 0.009 between CCM and Rad Sal. 

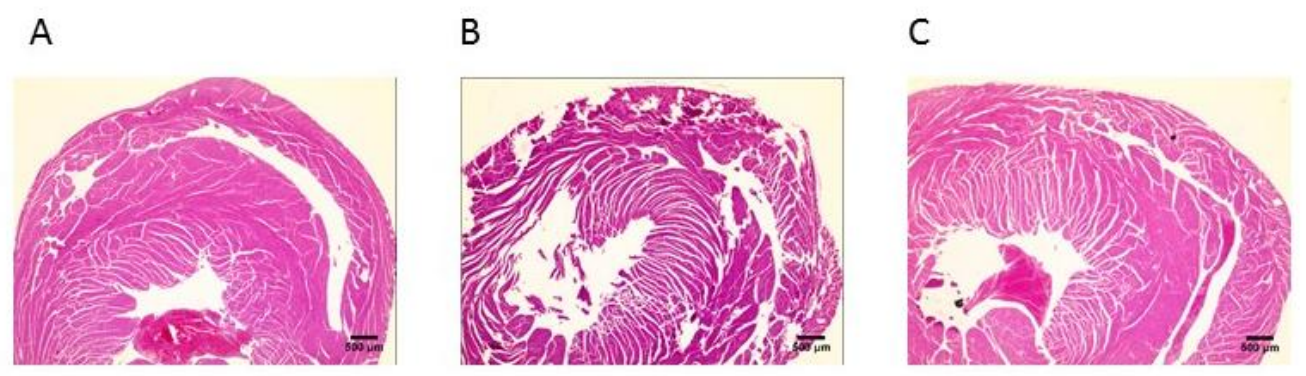

D

$\mathrm{E}$
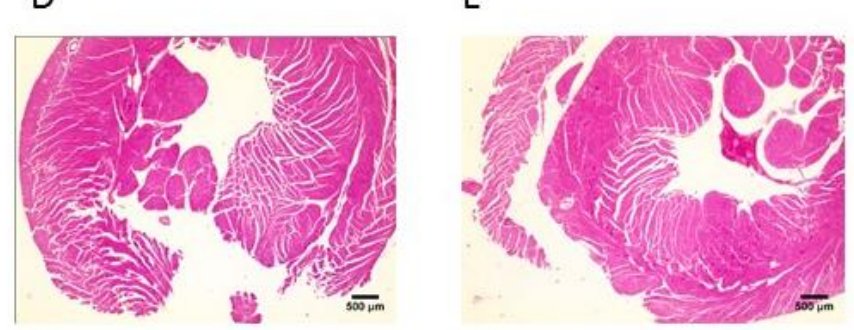

$\mathrm{F}$

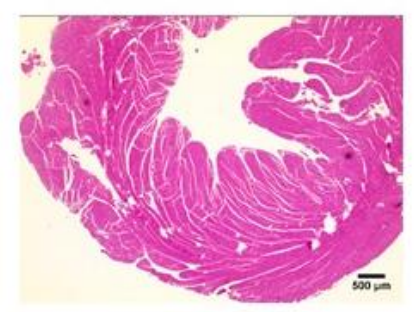

FIGURE 22: Representative images for the wall thickness of the right ventricle. A: NR Sal, B: Rad Sal. C: Chitocurc. D: Lipids. E: Alb/CCM. F: CCM

A

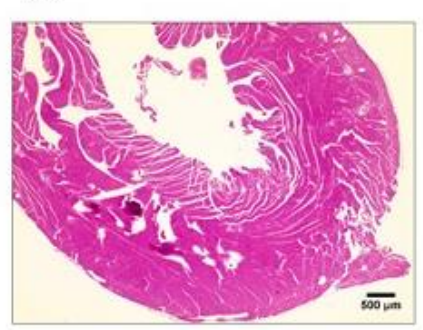

D

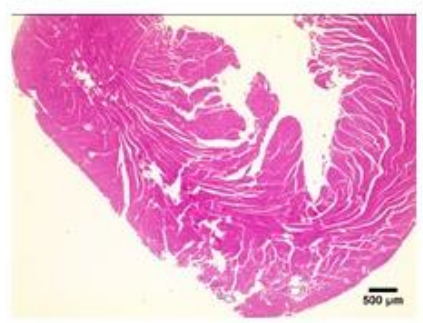

B

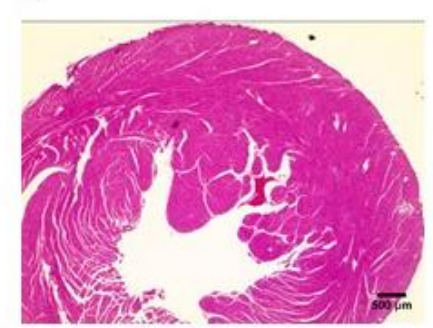

$\mathrm{E}$

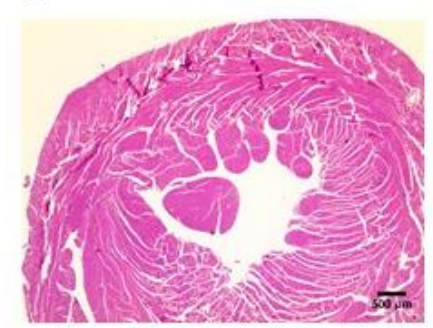

C

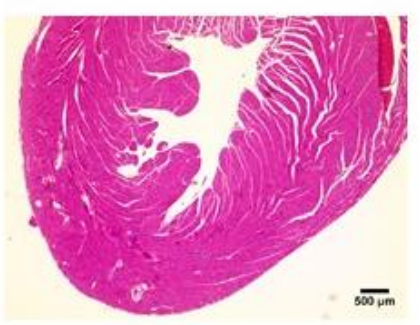

$\mathrm{F}$

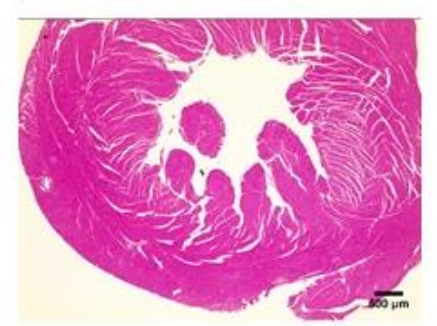

FIGURE 23: Representative images for the wall thickness of the left ventricle. A: NR Sal, B: Rad Sal. C: Chitocurc. D: Lipids. E: Alb/CCM. F: CCM 


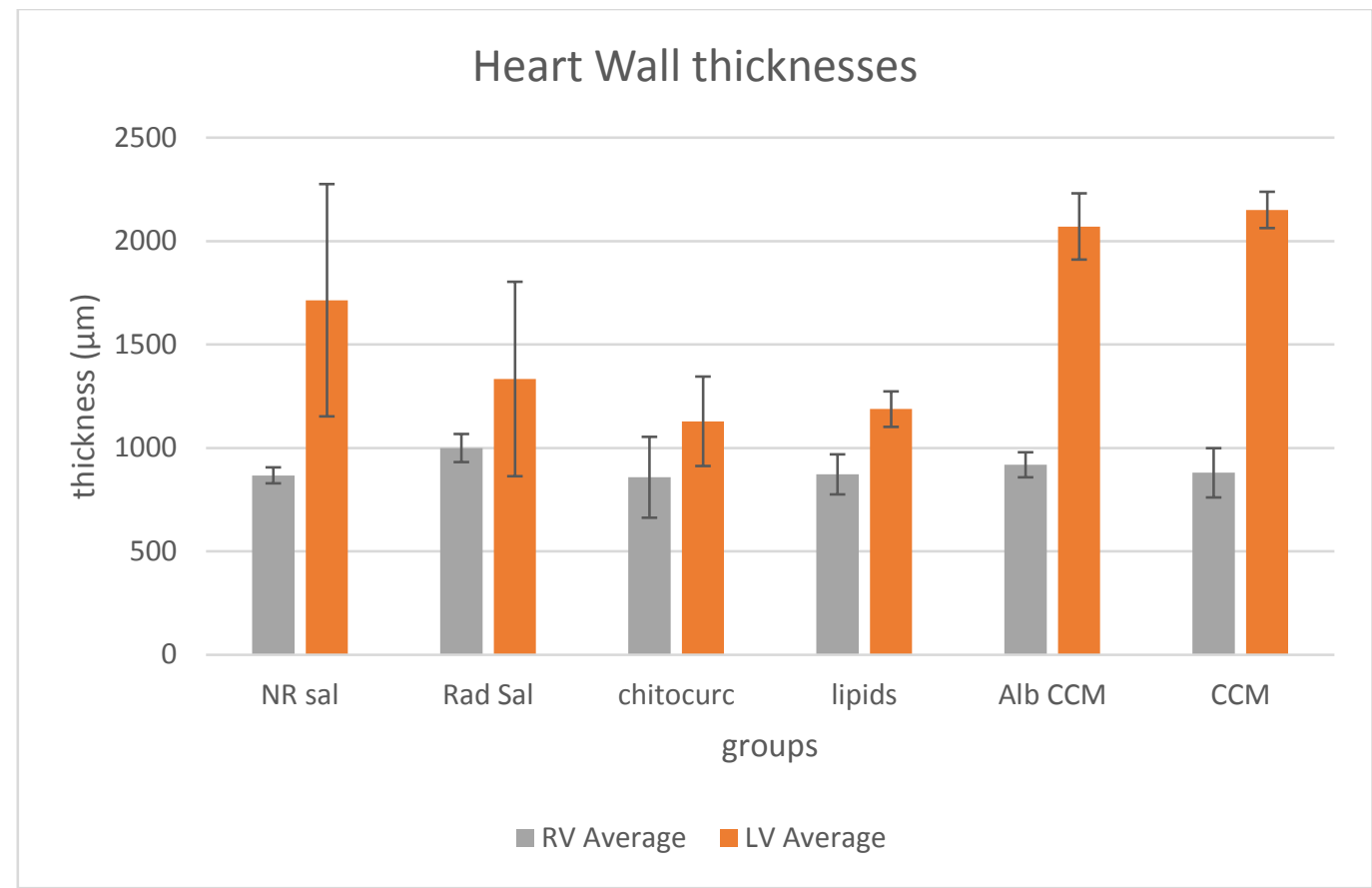

FIGURE 24: Graph of the wall thicknesses of the right and left ventricle as measured from the H\&E stained slides.

\section{Histology - Masson's Trichrome}

Masson's Trichrome is a three color stain that stains smooth muscle dark pink/red, ECM

light blue, and nuclei dark blue. In this case this would allow the amount of collagen to be quantified in ImageJ, since collagen is a major marker of fibrosis. In this case, however, the collagen made up less than $1 \%$ of the total area in each ventricle as can be seen in Figure 25 below which shows representative images of the left ventricle. The collagen would show up blue, but little if any blue can be seen in the images below. The graph indicating the levels of collagen in the left and right ventricles is in Figure 26 below. Since the highest level of collagen was still below $1 \%$, it can be concluded that increased collagen levels associated with fibrosis was not observed in this study. These results contrast with those of a study by Zeng et al. [48] which studied the effects of radiation induced damage of the myocardium with gamma radiation. In that study, there was an increase in collagen deposition in the myocardium after the 15 and 22 
Gy radiation and as much as a 5 fold increase in collagen compared to the non-radiated mice. In the Zeng et al. study, however, the mice were given much larger doses of radiation compared to the study here as well as sacrificing the mice after 4 months rather than 2 weeks.
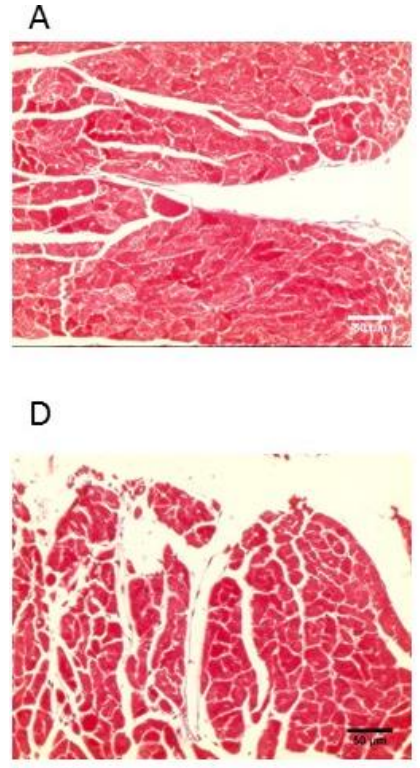

B

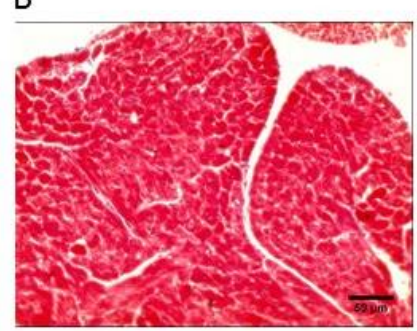

$E$

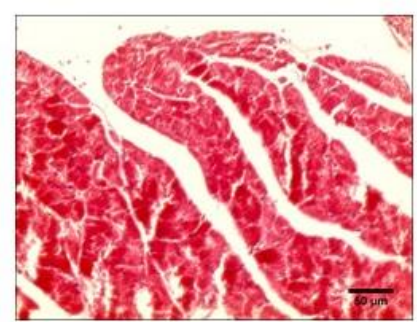

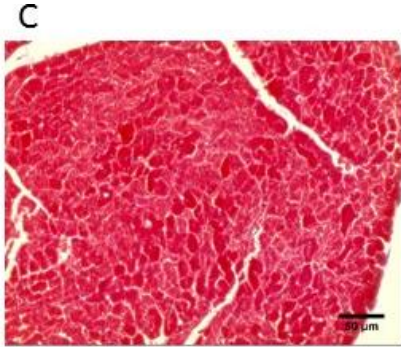

$\mathrm{F}$

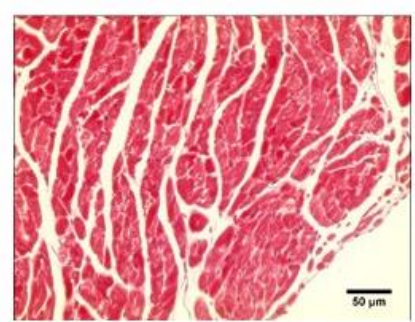

FIGURE 25: Representative images of the collagen amount found in the left ventricle of the hearts. A: NR Sal, B: Rad Sal. C: Chitocurc. D: Lipids. E: Alb/CCM. F: CCM

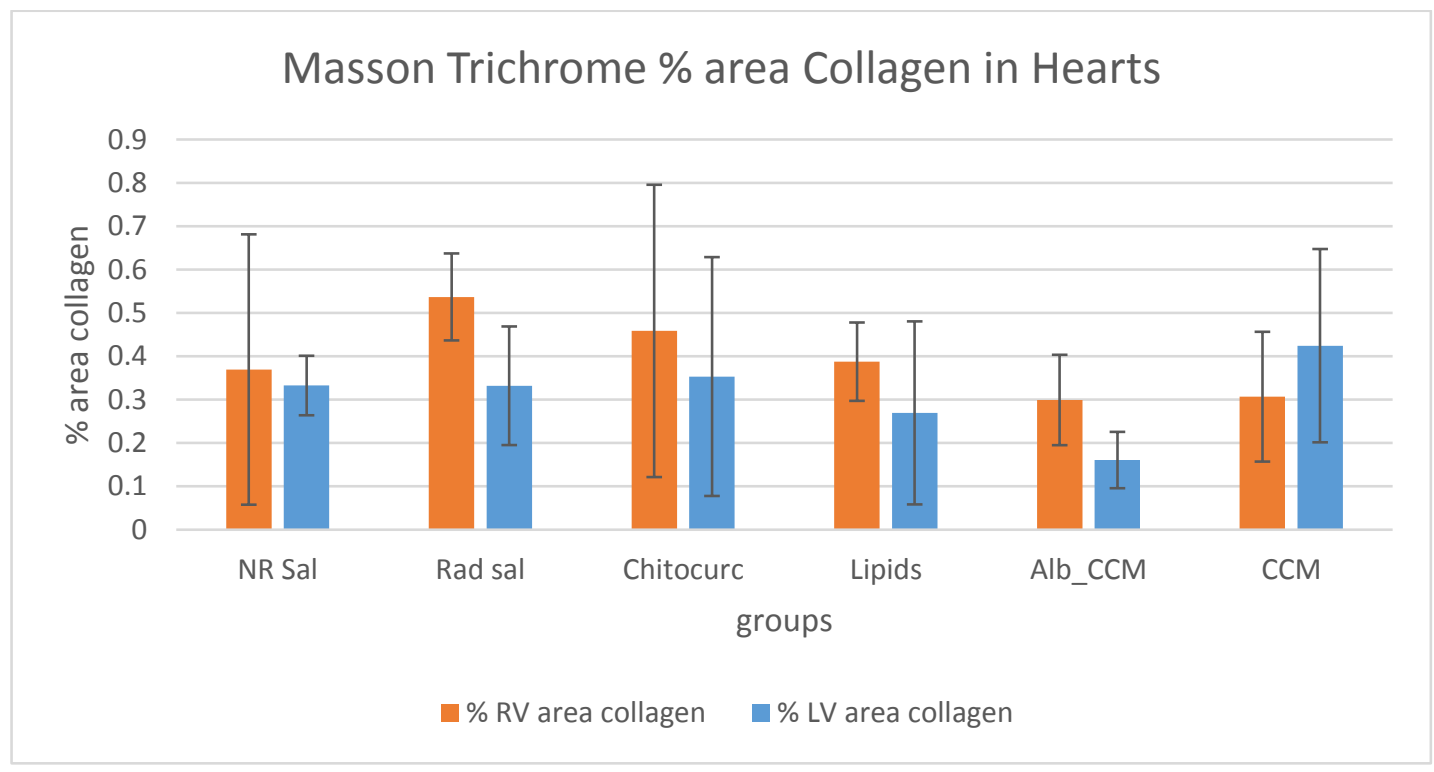

FIGURE 26: Graph of the amount of collagen found in the Masson's trichrome stained heart. 


\section{Histology - Immunostaining:}

The ventricles were also tested for TGF- $\beta 1$ via immunohistology since TGF- $\beta 1$ is linked to collagen deposition and cardiac fibrosis. The graph of the averages and standard deviations is below in Figure 29. The representative images are below in Figures 27 and 28. There was no significant effect of the radiation in the right or left ventricles comparing NR Sal and Rad Sal treatments, with $\mathrm{t}$ tests showing $\mathrm{p}$ values of 0.920 and 0.440 respectively. There were also no significant effects of the curcumin delivery vehicles in the right or left ventricles, as shown by ANOVAs with $p$ values of 0.620 and 0.894 respectively. While there were no significant differences, there were trends. For the right ventricle, there was almost no change based on the radiation, with a 4\% decrease in Rad Sal compared to NR Sal. The Chitocurc and CCM groups featured increases of $40 \%$ and $41 \%$ increases in TGF- $\beta 1$ compared to Rad Sal. The Alb/CCM treatment showed a 35\% decrease in TGF- $\beta 1$ compared to Rad Sal. For the left ventricle, there was a 55\% increase in TGF- $\beta 1$ in the Rad Sal treatment compared to NR Sal. There is a 37\% decrease in TGF- $\beta 1$ expression in the Alb/CCM group compared to Rad Sal. These trends could show that the Alb/CCM treatment does the most to reduce the expression of TGF- $\beta 1$, while Chitocurc and CCM may increase the expression of TGF- $\beta 1$, at least in the right ventricle. A study by Liu et al. using 20 Gy gamma radiation noted something more similar to the left ventricle results, with cardiac TGF- $\beta 1$ mRNA being expressed more than in the non-radiated group with peaks at 2 and 12 weeks [49]. The Liu et al. study used a polymerase chain reaction to quantify the TGF- $\beta 1$ as well as sacrificing at $2,4,8,12$, and 24 weeks rather than staining heart tissue to quantify and sacrifice after 2 weeks as was done in this study. Since Liu et al. quantified expression of mRNA rather that protein, it is possible that the peaks found by their mRNA studies at 2 weeks would not have translated into an increase in protein expression at that time point, similar to the observations found in this study. 

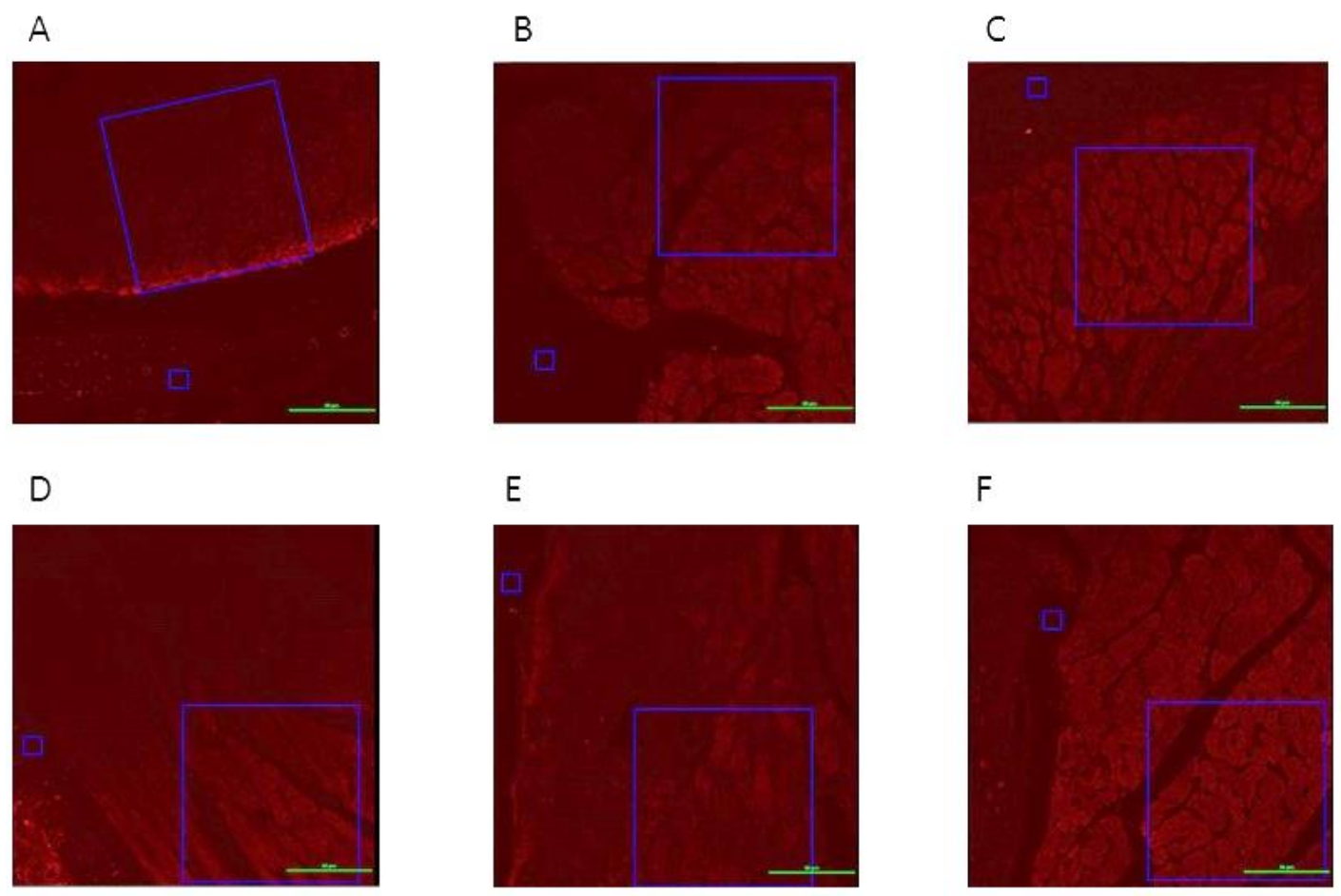

FIGURE 27: Representative images for the TGF- $\beta 1$ intensity of the right ventricle. A: NR Sal, B: Rad Sal. C: Chitocurc. D: Lipids. E: Alb/CCM. F: CCM. The representative images were all given stronger contrast in post processing to make the TGF- $\beta 1$ more visible. 

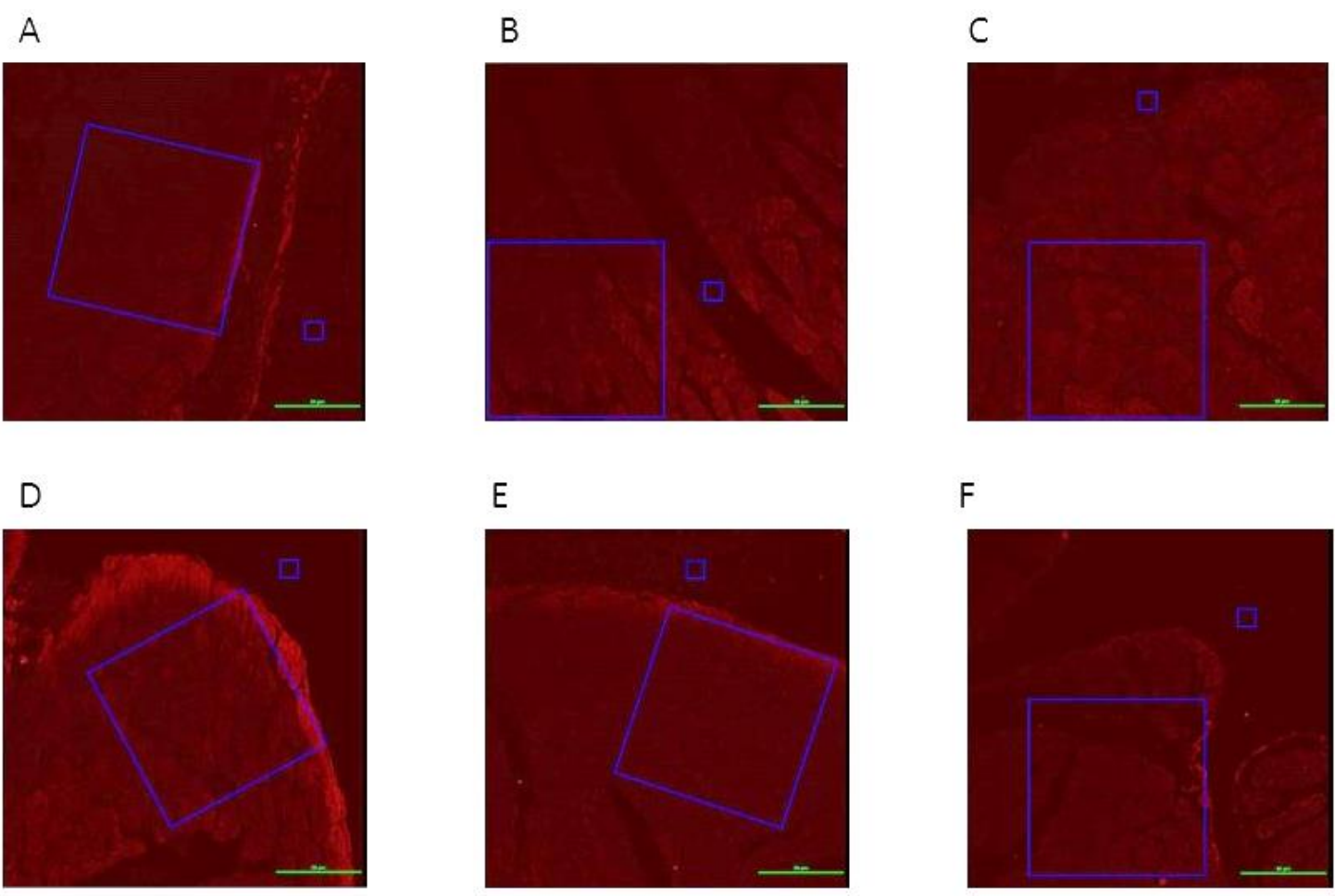

FIGURE 28: Representative images for the TGF- $\beta 1$ intensity of the left ventricle. A: NR Sal, B:

Rad Sal. C: Chitocurc. D: Lipids. E: Alb/CCM. F: CCM. The representative images were all given stronger contrast in post processing to make the TGF- $\beta 1$ more visible.

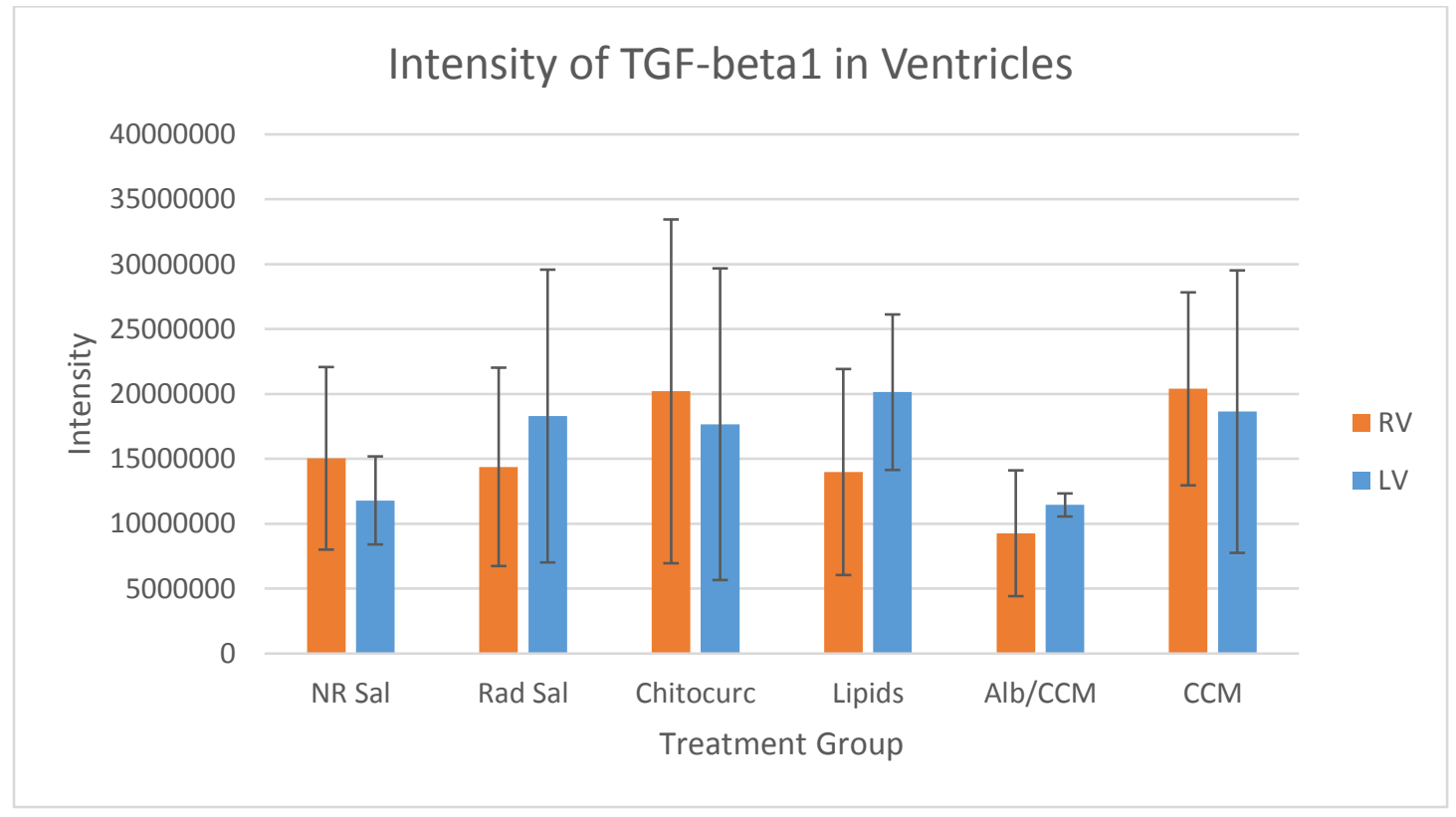

FIGURE 29: Graph of the intensity of TGF- $\beta 1$ in the immunofluorescent stained heart. 


\section{CONCLUSIONS, DISCUSSION, \& RECOMMENDATIONS FOR FUTURE WORK}

Conclusions:

Aortas:

Aim 1 was to characterize the effects of heavy iron radiation on mouse cardiovascular tissue. To test this, the Rad Sal and NR Sal treatment groups were compared in all of the previously mentioned assays using $t$ tests. The differences between the two were not significant in most of the tests, likely due to the low dose of radiation, type of radiation, and time point, but there were notable trends for each test. For the aortas, radiation seemed to increase in wall thickness compared to NR Sal, though the increase was variable. This thickening could result in stiffening of the vessel walls, which could lead to further cardiovascular problems or pathologies. Radiation exposure also increased the percent collagen in the aorta compared to NR Sal. This collagen increase would coincide with the vessel thickening, and with vessel stiffening documented by others.

Aim 2 was to examine the effectiveness of three independent curcumin drug delivery mechanisms at reducing mouse cardiovascular tissue damage caused by heavy iron radiation. In the case of the aortas, there were no significant differences shown by ANOVAs done in Minitab, but there were trends. For the aortic outgrowth assay, the Alb/CCM treatment showed the highest level of aortic outgrowth in mice compared to the other treatments, with a noticeable increase compared to Rad Sal. This would imply that the Alb/CCM treatment was most effective in promoting angiogenesis. In the wall thickness measurements, the Alb/CCM and Lipids treatments led to large decreases in wall thickness compared to Rad Sal, implying that these treatments helped to prevent radiation-induced stiffening. These results show that while not statistically significant, there were noticeable effects of the antioxidant treatments to the aorta. 
Each of the treatments had some effect counteracting the effects of radiation, with Alb/CCM showing the most positive countermeasure against the effects of radiation treatment.

\section{Ventricles:}

Aim 1 was to characterize the effects of heavy iron radiation on mouse cardiovascular tissue. To test this, the Rad Sal and NR Sal treatment groups were compared in all of the previously mentioned assays using $\mathrm{t}$ tests.

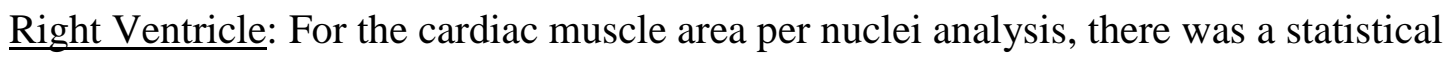
difference, with the radiation leading to a decrease in cardiac muscle area per nuclei comparing Rad Sal to NR Sal, indicating an increase in cells. For the wall thickness analysis, there was also a significant difference for the right ventricle, where the radiation appears to cause thickening of the right ventricle as signified by an increase in wall thickness in Rad Sal as compared to NR Sal. This thickening would lead to stiffening of the right ventricle, which may be an indication of cardiovascular disease.

Left Ventricle: For the cardiac muscle area per nuclei analysis the radiation led to a decrease in cardiac muscle area per nuclei comparing Rad Sal to NR Sal. For the wall thickness analysis, there was a trend with a decrease in left ventricular wall thickness as a result of radiation compared to NR Sal. This was not an expected result and bears further study, possibly indicating a thinning of the left ventricle that can occur before hypertrophy as shown by Sutton and Sharpe [50]. There could also be some effect elsewhere in the heart or arteries downstream leading to this thinning. There was an increase in TGF- $\beta 1$ left ventricle in the Rad Sal group compared to NR Sal. TGF- $\beta 1$ stimulates growth and could be there in an attempt to repair the thinning of the left ventricle. 
Aim 2 was to examine the effectiveness of three independent curcumin drug delivery mechanisms at reducing mouse cardiovascular tissue damage caused by heavy iron radiation.

Right Ventricle: For the cardiac muscle area per nuclei analysis, there was not a statistical difference between any of the antioxidant treatment groups and Rad Sal, nor were there any noticeable trends, signifying that there was no protective effect observed for this metric. For the wall thickness analysis all antioxidant treatments showed slight drops in wall thickness, with Chitocurc, Lipids, Alb/CCM, and CCM showing 14\%, 13\%, $8 \%$, and $12 \%$ decreases in wall thickness compared to Rad Sal. This would imply that all treatments provided some protective effect. When testing TGF- $\beta 1$ appearance in the heart, the Alb/CCM treatment showed a decrease in TGF- $\beta 1$ levels which would correspond with less fibrosis and the thinner right ventricles that were measured. Overall, these results show a small effect from all antioxidant treatments to mitigate the effects of radiation, with Alb/CCM showing the most effects.

Left Ventricle: For the cardiac muscle area per nuclei analysis, there was not a statistical difference between any of the antioxidant treatment groups and Rad Sal. For the wall thickness analysis, there was a significant effect between the treatment groups and Rad Sal. The radiation appeared to cause left ventricular thinning, and the Alb/CCM and CCM antioxidant treatments seemed to prevent this thinning, with increased in wall thickness. One explanation for the thinning could be stiffening of the vasculature prior to the left ventricle, since the aorta is not the only vessel that can be affected by radiation but was the only vessel investigated here. This work didn't measure stiffness but noted increased wall thickness and collagen content that could result in stiffening of the aorta. For the TGF- $\beta 1$ testing, there was a decrease in TGF- $\beta 1$ levels in the Alb/CCM treatment. Overall, these results show an ability on the part of Alb/CCM and CCM 
treatments to mitigate the effects of radiation induced cardiovascular damage in the left ventricle, especially Alb/CCM.

\section{Overall:}

The mice in this study were sacrificed two weeks after radiation. At that time point, the mice still appeared healthy, and did not have obvious signs of radiation damage. Based on literature, it is likely that a longer post-radiation study would have been indicated more conclusive radiation-induced cardiovascular damage. The significant effects shown as a result of radiation were a decrease in cardiac muscle per nuclei in the right ventricle and thickening of the right ventricle. Notable trends were also observed as a result of radiation, with thickening of the aortas and thinning of the left ventricle. These trends could have been more significant if larger sample sizes were used. The low amount of significant results reported here mirrors a paper by Heselich et al. in which heavy ion radiation (both titanium and iron) was used, and significant effects were not seen, indicating that single doses of radiation and short post-radiation sacrifices result in fewer significant observable effects [51]. In the Heselich paper, radiation led to DNA damage and a reduction of S-phase cells in cell cultures, hindering proliferation. Within 24 hours these cell cultures were able to repair DNA and recover S-phase cell levels, demonstrating that for radiation to cause long term effects it may need to be given repeatedly or over extended periods of time more like what happens in space.

Of the radiation effects noted here, Alb/CCM was the drug delivery vehicle that had a significant effect in mitigating changes in the left ventricle thickness. All of the treatments had some helpful effects, especially in reducing the changes in ventricular wall thickness, but $\mathrm{Alb} / \mathrm{CCM}$ was shown to have effects in all three tissue types, making it the most promising. 


\section{Limitations:}

There is inherent variability between mice, and this could not be eliminated because each mouse could only be given one of the drug delivery mechanisms. The mice could also not be tested before and after the radiation, since the tests required the mice to be sacrificed. As a result, the natural, pre-radiation differences between the mice could not be factored in to the study.

Another limitation was that the radiation dose administered to the mice was low, and cardiovascular diseases take time to develop. While some studies have shown doses as low as 50 cGy having an effect, most studies referenced here have used doses from 1-5 Gy [11, 27, 51]. A higher dose would likely show a more significant effect. The purpose of this work was to examine the effects of radiation exposure during spaceflight, thus a low dose of radiation was selected to mimic the small doses of radiation that astronauts are exposed to over the time of space travel. The mice were also sacrificed after two weeks which may not have been enough time for fibrosis to be observed.

\section{Future Work:}

Future work would include testing at higher doses of ${ }^{56} \mathrm{Fe}$ radiation and/or waiting longer periods of time before sacrifice of the mice to investigate long term. A higher sample size would be necessary for the different assays to determine significant effects from radiation exposure. Future work would also investigate the thinning of the left ventricle and any resulting cardiovascular disease resulting from it. Future work would also include trying antioxidants other than curcumin, due to some of curcumin's anti-proliferative effects. 


\section{REFERENCES}

1. White, C.R., et al., Heavy-ion (56Fe) irradiation leads to impaired aortic relaxation prior to atherosclerotic plaque formation in ApoE-/- mice. Journal of Radiation Research, 2014. 55(suppl_1): p. i42-i43.

2. Savage, D.E., et al., Radiation effects on left ventricular function and myocardial perfusion in long term survivors of Hodgkin's disease. International Journal of Radiation Oncology*Biology*Physics, 1990. 19(3): p. 721-727.

3. Hancock, S.L., M.A. Tucker, and R.T. Hoppe, Factors affecting late mortality from heart disease after treatment of hodgkin's disease. JAMA, 1993. 270(16): p. 1949-1955.

4. Gerber Thomas, C.T., lonizing radiation in cardiac imaging: a science advisory from the American Heart Association Committee on Cardiac Imaging of the Council on Clinical Cardiology and Committee on Cardiovascular Imaging and Intervention of the Council on Cardiovascular Radiology and Intervention. Circulation, 2009. 119(7): p. 1056-65.

5. Cardis, E., et al., Effects of Low Doses and Low Dose Rates of External Ionizing Radiation: Cancer Mortality among Nuclear Industry Workers in Three Countries. Radiation Research, 1995. 142(2): p. 117-132.

6. Muirhead, C.R., et al., Occupational radiation exposure and mortality: second analysis of the National Registry for Radiation Workers. Journal of Radiological Protection, 1999. 19(1): p. 3.

7. Delp Michael, D.M., Apollo Lunar Astronauts Show Higher Cardiovascular Disease Mortality: Possible Deep Space Radiation Effects on the Vascular Endothelium. Scientific reports, 2016. 6.

8. Cucinotta, F.A. and M. Durante, Cancer risk from exposure to galactic cosmic rays: implications for space exploration by human beings. The Lancet Oncology, 2006. 7(5): p. 431-435.

9. Kennedy, A.R., Biological Effects of Space Radiation and Development of Effective Countermeasures. Life sciences in space research, 2014. 1: p. 10-43.

10. Rask, J., et al., Space Faring: The Radiation Challenge Module 1. 2007.

11. Soucy, K.G., et al., HZE 56Fe-Ion Irradiation Induces Endothelial Dysfunction in Rat Aorta: Role of Xanthine Oxidase. Radiation Research, 2011. 176(4): p. 474-485.

12. Cucinotta, F.A., et al., Radiation dosimetry and biophysical models of space radiation effects. Gravitational and space biology bulletin : publication of the American Society for Gravitational and Space Biology, 2003. 16(2): p. 11-8.

13. Rask, J., et al., Space Faring: The Radiation Challenge Module 3. 2007.

14. Durante, M. and F.A. Cucinotta, Heavy ion carcinogenesis and human space exploration. Nature Reviews Cancer, 2008. 8: p. 465.

15. Finkel, T. and N.J. Holbrook, Oxidants, oxidative stress and the biology of ageing. Nature, 2000. 408: p. 239.

16. Murphy, Michael P., How mitochondria produce reactive oxygen species. Biochemical Journal, 2009. 417(1): p. 1.

17. Jastroch, M., et al., Mitochondrial proton and electron leaks. Essays in biochemistry, 2010. 47: p. 53-67.

18. Finkel, T., Oxygen radicals and signaling. Current Opinion in Cell Biology, 1998. 10(2): p. 248253.

19. Azzam, E.I., J.-P. Jay-Gerin, and D. Pain, lonizing radiation-induced metabolic oxidative stress and prolonged cell injury. Cancer letters, 2012. 327(0): p. 48-60.

20. Richter, K., et al., Redox-fibrosis: Impact of TGFB1 on ROS generators, mediators and functional consequences. Redox Biology, 2015. 6(Supplement C): p. 344-352.

21. Westbury, C.B. and J.R. Yarnold, Radiation Fibrosis - Current Clinical and Therapeutic Perspectives. Clinical Oncology, 2012. 24(10): p. 657-672.

22. Leask, A. and D. Abraham, TGF beta Signaling and the fibrotic response. Vol. 18. 2004. 816-27. 
23. F Jobling, M., et al., Isoform-Specific Activation of Latent Transforming Growth Factor ?? (LTGF-??) by Reactive Oxygen Species. Vol. 166. 2007. 839-48.

24. Barcellos-Hoff, M., C. Park, and E. G Wright, Radiation and the microenvironment Tumorigenesis and therapy. Vol. 5. 2005. 867-75.

25. Ruiz-Ortega, M., et al., TGF-B signaling in vascular fibrosis. Vol. 74. 2007. 196-206.

26. Stewart, F.A., S. Hoving, and N.S. Russell, Vascular Damage as an Underlying Mechanism of Cardiac and Cerebral Toxicity in Irradiated Cancer Patients. Radiation Research, 2010. 174(6): p. 865-869.

27. Soucy, K.G., et al., Single exposure gamma-irradiation amplifies xanthine oxidase activity and induces endothelial dysfunction in rat aorta. Radiation and Environmental Biophysics, 2007. 46(2): p. 179-186.

28. Russell, N.S., et al., Novel insights into pathological changes in muscular arteries of radiotherapy patients. Radiotherapy and Oncology, 2009. 92(3): p. 477-483.

29. Giam Beverly, B., N-acetylcysteine attenuates the development of cardiac fibrosis and remodeling in a mouse model of heart failure. Physiological reports. 4(7).

30. Sharma, R.A., A.J. Gescher, and W.P. Steward, Curcumin: The story so far. European Journal of Cancer, 2005. 41(13): p. 1955-1968.

31. Anand, P., et al., Bioavailability of Curcumin: Problems and Promises. Molecular Pharmaceutics, 2007. 4(6): p. 807-818.

32. Aggarwal, B.B. and K.B. Harikumar, Potential Therapeutic Effects of Curcumin, the Antiinflammatory Agent, Against Neurodegenerative, Cardiovascular, Pulmonary, Metabolic, Autoimmune and Neoplastic Diseases. The international journal of biochemistry \& cell biology, 2009. 41(1): p. 40-59.

33. Hatcher, H., et al., Curcumin: From ancient medicine to current clinical trials. Cellular and Molecular Life Sciences, 2008. 65(11): p. 1631-1652.

34. HSIEH, C.-Y., Phase I clinical trial of curcumin, a chemopreventive agent, in patients with high-risk or pre-malignant lesions. Anticancer research, 2001. 21: p. 2895-2900.

35. O'Toole Martin, G.M., Release-Modulated Antioxidant Activity of a Composite CurcuminChitosan Polymer. Biomacromolecules, 2016. 17(4): p. 1253-60.

36. Liu, A., et al., Validated LC/MS/MS assay for curcumin and tetrahydrocurcumin in rat plasma and application to pharmacokinetic study of phospholipid complex of curcumin. Journal of Pharmaceutical and Biomedical Analysis, 2006. 40(3): p. 720-727.

37. Kim, T.H., et al., Preparation and characterization of water-soluble albumin-bound curcumin nanoparticles with improved antitumor activity. International Journal of Pharmaceutics, 2011. 403(1): p. 285-291.

38. Barik, A., et al., Interaction of curcumin with human serum albumin: Thermodynamic properties, fluorescence energy transfer and denaturation effects. Chemical Physics Letters, 2007. 436(1): p. 239-243.

39. Kunwar, A., et al., Absorption and fluorescence studies of curcumin bound to liposome and living cells. Vol. 285. 2007.

40. Soucy, K.G., et al., Single exposure to radiation produces early anti-angiogenic effects in mouse aorta. Radiation and Environmental Biophysics, 2010. 49(3): p. 397-404.

41. Templin Thomas, T., Proton radiation-induced miRNA signatures in mouse blood: characterization and comparison with $56 \mathrm{Fe}$-ion and gamma radiation. International Journal of Radiation Biology. 88(7): p. 531-9.

42. Baluchamy Sudhakar, S., Reactive oxygen species mediated tissue damage in high energy proton irradiated mouse brain. Molecular and Cellular Biochemistry. 360(1-2): p. 189-95. 
43. Peña, L.A., Z. Fuks, and R.N. Kolesnick, Radiation-induced Apoptosis of Endothelial Cells in the Murine Central Nervous System: Protection by Fibroblast Growth Factor and Sphingomyelinase Deficiency. Cancer Research, 2000. 60(2): p. 321.

44. Panchatcharam, M., et al., Curcumin improves wound healing by modulating collagen and decreasing reactive oxygen species. Molecular and Cellular Biochemistry, 2006. 290(1): p. 87-96.

45. Akbik, D., et al., Curcumin as a wound healing agent. Life Sciences, 2014. 116(1): p. 1-7.

46. Arbiser, J.L., et al., Curcumin is an in vivo inhibitor of angiogenesis. Molecular Medicine, 1998. 4(6): p. 376-383.

47. $\mathrm{Yu}, \mathrm{T}$., et al., Iron-Ion Radiation Accelerates Atherosclerosis in Apolipoprotein E-Deficient Mice. Radiation Research, 2011. 175(6): p. 766-773.

48. Zeng, Y.-C., et al., Sestrin2 protects the myocardium against radiation-induced damage. Radiation and Environmental Biophysics, 2016. 55(2): p. 195-202.

49. Liu, H., et al., [Correlation of TGF-beta1 mRNA expression to irradiation-induced heart damage in rats]. Vol. 27. 2008. 18-24.

50. Sutton, M.G.S.J. and N. Sharpe, Left Ventricular Remodeling After Myocardial Infarction. Circulation, 2000. 101(25): p. 2981.

51. Heselich, A., et al., High LET Radiation Shows no Major Cellular and Functional Effects on Primary Cardiomyocytes in Vitro. Life Sciences in Space Research, 2018. 
VITA

Alexandra DeCarlo London, KY 40744 | alliedecarlo@gmail.com

\section{EDUCATION}

\section{M.Eng in Bioengineering}

J.B Speed School of Engineering UofL_April 2018

B.S. in Bioengineering, summa cum lade

J.B. Speed School of Engineering UofL_May 2016

\section{EMPLOYMENT}

enmodes GmbH - Aachen, Germany May - August 2015, January-April 2016

Cooperative Education Student Employee

- Testing of devices - flow loops and pressure tests

- Compared device results with existing products and created graphs in Excel

- Device assembly and manufacture

- Observed and recorded live animal testing

- Basic designing in Creo Parametric for 3D printed parts

- Produced summaries of existing research and did research on existing patents

Boston Scientific - Spencer, Indiana

August - December 2014

Cooperative Education Student Employee

- Tested medical devices using Instron, Smartscope, LazerMike

- Trained in company protocol regarding paperwork, review, and quality control

- Worked in teams to achieve project goals.

- Created test methods and technical reports

HONORS AND AWARDS

- Alfred T. Chen Memorial Scholarship Award

- Jerry And Pat Sturgeon Academic Excellence Award

- Dean's List 


\title{
Student $\mathbf{t}$ tests
}

Aortic Outgrowth

\section{Two-Sample T-Test and CI: Saline Outgrowth, Saline Treatments}

\author{
Method \\ $\mu_{1}$ : mean of Saline Outgrowth when Saline Treatments $=\mathrm{nr}$ sal \\ $\mu_{2}$ : mean of Saline Outgrowth when Saline Treatments $=$ rad sal \\ Difference: $\mu_{1}-\mu_{2}$ \\ Equal variances are not assumed for this analysis.
}

Descriptive Statistics: Saline Outgrowth

Saline

\begin{tabular}{lrrrr} 
Treatments & N & Mean & StDev & SE Mean \\
\hline nr sal & 5 & 5847 & 4786 & 2140 \\
rad sal & 7 & 7649 & 4552 & 1721
\end{tabular}

Estimation for Difference

\begin{tabular}{rc} 
& $95 \%$ CI for \\
Difference & Difference \\
\hline-1802 & $(-8135,4530)$
\end{tabular}

\section{Test}

Null hypothesis $\quad H_{0}: \mu_{1}-\mu_{2}=0$

Alternative hypothesis $H_{1}: \mu_{1}-\mu_{2} \neq 0$

\begin{tabular}{rrr} 
T-Value & DF & P-Value \\
\hline-0.66 & 8 & 0.530
\end{tabular}

ROS ASSAY

\section{Two-Sample T-Test and CI: Saline ROS, Saline Treatments}

\section{Method \\ $\mu_{1}$ : mean of Saline ROS when Saline Treatments $=$ NR Sal \\ $\mu_{2}$ : mean of Saline ROS when Saline Treatments $=$ Rad Sal \\ Difference: $\mu_{1}-\mu_{2}$}

Equal variances are not assumed for this analysis.

Descriptive Statistics: Saline ROS 
Saline

\begin{tabular}{lrrrr} 
Treatments & N & Mean & StDev & SE Mean \\
\hline NR Sal & 2 & 0.0548 & 0.0244 & 0.017 \\
Rad Sal & 3 & 0.04817 & 0.00580 & 0.0033
\end{tabular}

Estimation for Difference

\begin{tabular}{rc}
$95 \%$ CI for \\
Difference & Difference \\
\hline 0.0066 & $(-0.2167,0.2299)$
\end{tabular}

\section{Test}

Null hypothesis $\quad H_{0}: \mu_{1}-\mu_{2}=0$

Alternative hypothesis $H_{1}: \mu_{1}-\mu_{2} \neq 0$

\begin{tabular}{rrr} 
T-Value & DF & P-Value \\
\hline 0.37 & 1 & 0.772
\end{tabular}

\section{$H \& E$}

Aorta

\section{Two-Sample T-Test and CI: Saline Muscle Tissue/Nuclei ... ents Aorta}

\section{Method}

$\mu_{1}$ : mean of Saline Muscle Tissue/Nuclei Aor when Saline Treatments Aorta $=$ NR sal $\mu_{2}$ : mean of Saline Muscle Tissue/Nuclei Aor when Saline Treatments Aorta $=$ Rad sal Difference: $\mu_{1}-\mu_{2}$

Equal variances are not assumed for this analysis.

Descriptive Statistics: Saline Muscle Tissue/Nuclei Aor

\begin{tabular}{lrrrr}
$\begin{array}{l}\text { Saline } \\
\text { Treatments }\end{array}$ & & & & \\
Aorta & N & Mean & StDev & $\begin{array}{r}\text { SE } \\
\text { Mean }\end{array}$ \\
\hline NR sal & 2 & 204.6 & 23.0 & 16 \\
Rad sal & 3 & 191.7 & 57.3 & 33
\end{tabular}

\section{Estimation for Difference}

\begin{tabular}{rc} 
& $95 \%$ CI for \\
Difference & Difference \\
\hline 12.9 & $(-145.7,171.6)$
\end{tabular}


Test

Null hypothesis $\quad \mathrm{H}_{0}: \mu_{1}-\mu_{2}=0$

Alternative hypothesis $H_{1}: \mu_{1}-\mu_{2} \neq 0$

\begin{tabular}{rrr} 
T-Value & DF & P-Value \\
\hline 0.35 & 2 & 0.760
\end{tabular}

Right Ventricle

\section{Two-Sample T-Test and CI: Saline Muscle Tissue/Nuclei ... atments RV}

\section{Method}

$\mu_{1}$ : mean of Saline Muscle Tissue/Nuclei RV when Saline Treatments RV = NR Sal

$\mu_{2}$ : mean of Saline Muscle Tissue/Nuclei RV when Saline Treatments RV = Rad Sal

Difference: $\mu_{1}-\mu_{2}$

Equal variances are not assumed for this analysis.

\section{Descriptive Statistics: Saline Muscle Tissue/Nuclei RV}

Saline

Treatments SE

\begin{tabular}{lrrrr} 
RV & N & Mean & StDev & Mean \\
\hline NR Sal & 5 & 396.7 & 37.7 & 17
\end{tabular}

$\begin{array}{lllll}\text { Rad Sal } & 4 & 332.3 & 36.6 & 18\end{array}$

Estimation for Difference

\begin{tabular}{rr} 
& $95 \%$ CI for \\
Difference & Difference \\
\hline 64.4 & $(3.6,125.2)$
\end{tabular}

Test

Null hypothesis $\quad H_{0}: \mu_{1}-\mu_{2}=0$

Alternative hypothesis $H_{1}: \mu_{1}-\mu_{2} \neq 0$

\begin{tabular}{rrr} 
T-Value & DF & P-Value \\
\hline 2.59 & 6 & 0.041
\end{tabular}

Left Ventricle 


\title{
Two-Sample T-Test and CI: Saline Muscle Tissue/Nuclei ... atments LV
}

\author{
Method \\ $\mu_{1}$ : mean of Saline Muscle Tissue/Nuclei LV when Saline Treatments LV = NR Sal \\ $\mu_{2}$ : mean of Saline Muscle Tissue/Nuclei LV when Saline Treatments LV $=$ Rad Sal \\ Difference: $\mu_{1}-\mu_{2}$
}

Equal variances are not assumed for this analysis.

Descriptive Statistics: Saline Muscle Tissue/Nuclei LV

\begin{tabular}{lrrrr}
$\begin{array}{l}\text { Saline } \\
\text { Treatments } \\
\text { LV }\end{array}$ & & & & \\
\hline NR Sal & N & Mean & StDev & $\begin{array}{r}\text { SE } \\
\text { Mean }\end{array}$ \\
Rad Sal & 4 & 322.0 & 40.9 & 18 \\
& 4 & 50.1 & 25
\end{tabular}

Estimation for Difference

\begin{tabular}{rc} 
& $95 \%$ CI for \\
Difference & Difference \\
\hline 19.2 & $(-60.5,99.0)$
\end{tabular}

Test

Null hypothesis $\quad \mathrm{H}_{0}: \mu_{1}-\mu_{2}=0$

Alternative hypothesis $H_{1}: \mu_{1}-\mu_{2} \neq 0$

\begin{tabular}{rrr} 
T-Value & DF & P-Value \\
\hline 0.62 & 5 & 0.562
\end{tabular}

Thickness Measurements

Aorta

Two-Sample T-Test and CI: Saline Thickness Aorta, Saline ... ents Aorta

\author{
Method \\ $\mu_{1}$ : mean of Saline Thickness Aorta when Saline Treatments Aorta $=$ NR sal \\ $\mu_{2}$ : mean of Saline Thickness Aorta when Saline Treatments Aorta $=$ Rad Sal \\ Difference: $\mu_{1}-\mu_{2}$ \\ Equal variances are not assumed for this analysis.
}


Descriptive Statistics: Saline Thickness Aorta

\begin{tabular}{lrrrr}
$\begin{array}{l}\text { Saline } \\
\text { Treatments }\end{array}$ & & & & \\
Aorta & $N$ & Mean & StDev & SE Mean \\
\hline NR sal & 2 & 58.583 & 0.353 & 0.25 \\
Rad Sal & 3 & 81.6 & 24.1 & 14
\end{tabular}

Estimation for Difference

\begin{tabular}{rc} 
& $95 \%$ CI for \\
Difference & Difference \\
\hline-23.0 & $(-82.7,36.8)$
\end{tabular}

\section{Test}

Null hypothesis $\quad \mathrm{H}_{0}: \mu_{1}-\mu_{2}=0$

Alternative hypothesis $\mathrm{H}_{1}: \mu_{1}-\mu_{2} \neq 0$

\begin{tabular}{rrr} 
T-Value & DF & P-Value \\
\hline-1.66 & 2 & 0.240
\end{tabular}

Right Ventricle

\section{Two-Sample T-Test and CI: Saline Thickness RV, Saline Treatments RV}

\section{Method}

$\mu_{1}$ : mean of Saline Thickness RV when Saline Treatments RV $=$ NR Sal

$\mu_{2}$ : mean of Saline Thickness RV when Saline Treatments RV = Rad Sal

Difference: $\mu_{1}-\mu_{2}$

Equal variances are not assumed for this analysis.

Descriptive Statistics: Saline Thickness RV

Saline

Treatments SE

\begin{tabular}{lrrrr} 
RV & N & Mean & StDev & Mean \\
\hline NR Sal & 5 & 867.6 & 38.7 & 17
\end{tabular}

$\begin{array}{lllll}\text { Rad Sal } \quad 4 & 999.6 & 67.9 & 34\end{array}$

Estimation for Difference 


\begin{tabular}{rc} 
& $95 \%$ CI for \\
Difference & Difference \\
\hline$-132.0 \quad(-237.8,-26.2)$
\end{tabular}

\title{
Test
}

Null hypothesis $\quad H_{0}: \mu_{1}-\mu_{2}=0$

Alternative hypothesis $H_{1}: \mu_{1}-\mu_{2} \neq 0$

T-Value DF P-Value

$\begin{array}{lll}-3.47 & 4 & 0.026\end{array}$

Left Ventricle

\section{Two-Sample T-Test and CI: Saline Thickness LV, Saline Treatments LV}

\author{
Method \\ $\mu_{1}$ : mean of Saline Thickness LV when Saline Treatments LV $=$ NR Sal \\ $\mu_{2}$ : mean of Saline Thickness LV when Saline Treatments LV $=$ Rad Sal \\ Difference: $\mu_{1}-\mu_{2}$
}

Equal variances are not assumed for this analysis.

\section{Descriptive Statistics: Saline Thickness LV}

Saline

Treatments

\begin{tabular}{lrrrr} 
LV & N & Mean & StDev & SE Mean \\
\hline NR Sal & 5 & 1714 & 561 & 251 \\
Rad Sal & 4 & 1333 & 543 & 271
\end{tabular}

\section{Estimation for Difference}

\begin{tabular}{rc} 
& $95 \%$ CI for \\
Difference & Difference \\
\hline 381 & $(-524,1285)$
\end{tabular}

\section{Test}

Null hypothesis $\quad H_{0}: \mu_{1}-\mu_{2}=0$

Alternative hypothesis $H_{1}: \mu_{1}-\mu_{2} \neq 0$

T-Value DF P-Value

$\begin{array}{lll}1.03 & 6 & 0.343\end{array}$

Masson Trichrome 


\title{
Two-Sample T-Test and CI: Adventitia Thickness ... rta Thickness Radia
}

\author{
Method \\ $\mu_{1}$ : mean of Adventitia Thickness Radiation when Treatment Aorta Thickness Radia $=$ NR Sal \\ $\mu_{2}$ : mean of Adventitia Thickness Radiation when Treatment Aorta Thickness Radia $=$ Rad Sal \\ Difference: $\mu_{1}-\mu_{2}$ \\ Equal variances are not assumed for this analysis.
}

Descriptive Statistics: Adventitia Thickness Radiation

Treatment

Aorta

Thickness

\begin{tabular}{lrrrr} 
Radia & N & Mean & StDev & SE Mean \\
\hline NR Sal & 2 & 10.42 & 6.76 & 4.8
\end{tabular}

$\begin{array}{lllll}\text { Rad Sal } \quad 3 & 16.32 & 9.27 & 5.4\end{array}$

Estimation for Difference

\begin{tabular}{rc} 
& $95 \%$ CI for \\
Difference & Difference \\
\hline-5.90 & $(-36.78,24.98)$
\end{tabular}

\section{Test}

Null hypothesis $\quad \mathrm{H}_{0}: \mu_{1}-\mu_{2}=0$

Alternative hypothesis $H_{1}: \mu_{1}-\mu_{2} \neq 0$

\begin{tabular}{rrr} 
T-Value & DF & P-Value \\
\hline-0.82 & 2 & 0.498
\end{tabular}

\section{Two-Sample T-Test and CI: \%Adventitia of total ... rta Thickness Radia}

\author{
Method \\ $\mu_{1}$ : mean of \%Adventitia of total thicknes_1 when Treatment Aorta Thickness Radia = NR Sal \\ $\mu_{2}$ : mean of \%Adventitia of total thicknes_1 when Treatment Aorta Thickness Radia $=$ Rad \\ Sal \\ Difference: $\mu_{1}-\mu_{2}$ \\ Equal variances are not assumed for this analysis.
}




\section{Descriptive Statistics: \%Adventitia of total thicknes_1}

Treatment

Aorta

Thickness

\begin{tabular}{lrrrr} 
Radia & N & Mean & StDev & SE Mean \\
\hline NR Sal & 2 & 15.09 & 8.46 & 6.0
\end{tabular}

$\begin{array}{lllll}\text { Rad Sal } & 3 & 18.00 & 4.84 & 2.8\end{array}$

\section{Estimation for Difference}

\begin{tabular}{rc} 
& $95 \%$ CI for \\
Difference & Difference \\
\hline-2.91 & $(-86.78,80.97)$
\end{tabular}

\section{Test}

Null hypothesis $\quad \mathrm{H}_{0}: \mu_{1}-\mu_{2}=0$

Alternative hypothesis $H_{1}: \mu_{1}-\mu_{2} \neq 0$

\begin{tabular}{rrr} 
T-Value & DF & P-Value \\
\hline-0.44 & 1 & 0.736
\end{tabular}

\section{Two-Sample T-Test and CI: Saline \% collagen Aorta, ... eatments Aorta}

\section{Method}

$\mu_{1}$ : mean of Saline $\%$ collagen Aorta when Saline Treatments Aorta $=$ NR Sal $\mu_{2}$ : mean of Saline \% collagen Aorta when Saline Treatments Aorta $=$ Rad Sal

Difference: $\mu_{1}-\mu_{2}$

Equal variances are not assumed for this analysis.

Descriptive Statistics: Saline \% collagen Aorta

Saline

Treatments

\begin{tabular}{lrrrr} 
Aorta & N & Mean & StDev & SE Mean \\
\hline NR Sal & 2 & 8.82 & 7.81 & 5.5 \\
Rad Sal & 3 & 10.839 & 0.246 & 0.14
\end{tabular}

\section{Estimation for Difference}

\begin{tabular}{ll}
$95 \% \mathrm{CI}$ for \\
Difference & Difference \\
\hline
\end{tabular}




\section{Test}

Null hypothesis $\quad H_{0}: \mu_{1}-\mu_{2}=0$

Alternative hypothesis $H_{1}: \mu_{1}-\mu_{2} \neq 0$

$\begin{array}{rrr}\text { T-Value } & \text { DF } & \text { P-Value } \\ -0.37 & 1 & 0.777\end{array}$

Right Ventricle

\section{Two-Sample T-Test and CI: Saline \% collagen RV, Saline ... atments RV}

\section{Method}

$\mu_{1}$ : mean of Saline \% collagen RV when Saline Treatments RV = NR Sal

$\mu_{2}$ : mean of Saline \% collagen RV when Saline Treatments RV $=$ Rad Sal

Difference: $\mu_{1}-\mu_{2}$

Equal variances are not assumed for this analysis.

Descriptive Statistics: Saline \% collagen RV

\begin{tabular}{lrrrr}
$\begin{array}{l}\text { Saline } \\
\text { Treatments }\end{array}$ & & & & \\
RV & $N$ & Mean & StDev & SE Mean \\
\hline NR Sal & 3 & 0.370 & 0.312 & 0.18 \\
Rad Sal & 3 & 0.537 & 0.100 & 0.058
\end{tabular}

Estimation for Difference

\begin{tabular}{rc} 
& $\begin{array}{c}95 \% \text { CI for } \\
\text { Difference }\end{array}$ \\
\hline-0.167 & $(-0.981,0.646)$
\end{tabular}

\section{Test}

Null hypothesis $\quad \mathrm{H}_{0}: \mu_{1}-\mu_{2}=0$

Alternative hypothesis $H_{1}: \mu_{1}-\mu_{2} \neq 0$

\begin{tabular}{rrr} 
T-Value & DF & P-Value \\
\hline-0.89 & 2 & 0.469
\end{tabular}

Left Ventricle 


\title{
Two-Sample T-Test and CI: Saline \%collagen LV, Saline Treatments LV
}

\author{
Method \\ $\mu_{1}$ : mean of Saline \%collagen LV when Saline Treatments LV $=$ NR Sal \\ $\mu_{2}$ : mean of Saline \%collagen LV when Saline Treatments LV = Rad Sal \\ Difference: $\mu_{1}-\mu_{2}$
}

Equal variances are not assumed for this analysis.

Descriptive Statistics: Saline \%collagen LV

Saline

Treatments

\begin{tabular}{lrrrr} 
LV & N & Mean & StDev & SE Mean \\
\hline NR Sal & 3 & 0.3326 & 0.0687 & 0.040
\end{tabular}

$\begin{array}{lllll}\text { Rad Sal } & 3 & 0.332 & 0.137 & 0.079\end{array}$

Estimation for Difference

\begin{tabular}{rc}
$95 \%$ CI for \\
Difference & Difference \\
\hline 0.0005 & $(-0.3797,0.3808)$
\end{tabular}

\section{Test}

Null hypothesis $\quad \mathrm{H}_{0}: \mu_{1}-\mu_{2}=0$

Alternative hypothesis $H_{1}: \mu_{1}-\mu_{2} \neq 0$

$\begin{array}{rrr}\text { T-Value } & \text { DF } & \text { P-Value } \\ 0.01 & 2 & 0.996\end{array}$

\section{Immunohistology}

Aorta

Two-Sample T-Test and CI: Saline intensity Aorta, Saline ... ents Aorta

\author{
Method \\ $\mu_{1}$ : mean of Saline intensity Aorta when Saline Treatments Aorta $=$ NR Sal \\ $\mu_{2}$ : mean of Saline intensity Aorta when Saline Treatments Aorta $=$ Rad Sal
}


Difference: $\mu_{1}-\mu_{2}$

Equal variances are not assumed for this analysis.

Descriptive Statistics: Saline intensity Aorta

Saline

Treatments

\begin{tabular}{lrrrr} 
Aorta & N & Mean & StDev & SE Mean \\
\hline NR Sal & 2 & 1080704 & 509583 & 360329
\end{tabular}

Rad Sal $\quad 2 \quad 2750209 \quad 3208276 \quad 2268593$

Estimation for Difference

\begin{tabular}{cc} 
& $\begin{array}{l}95 \% \text { CI for } \\
\text { Difference }\end{array}$ \\
\hline-1669505 & $(-30856057,27517047)$
\end{tabular}

\section{Test}

Null hypothesis $\quad \mathrm{H}_{0}: \mu_{1}-\mu_{2}=0$

Alternative hypothesis $H_{1}: \mu_{1}-\mu_{2} \neq 0$

\begin{tabular}{rrr} 
T-Value & DF & P-Value \\
\hline-0.73 & 1 & 0.600
\end{tabular}

Right Ventricle

\section{Two-Sample T-Test and CI: Saline intensity RV, Saline Treatments RV}

\section{Method}

$\mu_{1}$ : mean of Saline intensity RV when Saline Treatments RV = NR Sal

$\mu_{2}$ : mean of Saline intensity RV when Saline Treatments RV $=$ Rad Sal

Difference: $\mu_{1}-\mu_{2}$

Equal variances are not assumed for this analysis.

Descriptive Statistics: Saline intensity RV

Saline

Treatments

\begin{tabular}{lrrrr} 
RV & N & Mean & StDev & SE Mean \\
\hline NR Sal & 3 & 15042834 & 7031889 & 4059863 \\
Rad Sal & 3 & 14385768 & 7641902 & 4412054
\end{tabular}




\section{Estimation for Difference}

\begin{tabular}{rc} 
& $\begin{array}{c}95 \% \text { CI for } \\
\text { Difference }\end{array}$ \\
\hline 657066 & $(-18424005,19738136)$
\end{tabular}

\section{Test}

Null hypothesis $\quad H_{0}: \mu_{1}-\mu_{2}=0$

Alternative hypothesis $\mathrm{H}_{1}: \mu_{1}-\mu_{2} \neq 0$

\begin{tabular}{rrr} 
T-Value & DF & P-Value \\
\hline 0.11 & 3 & 0.920
\end{tabular}

Left Ventricle

\section{Two-Sample T-Test and CI: Saline intensity LV, Saline Treatments LV}

\section{Method}

$\mu_{1}$ : mean of Saline intensity LV when Saline Treatments LV = NR Sal

$\mu_{2}$ : mean of Saline intensity LV when Saline Treatments LV $=$ Rad Sal

Difference: $\mu_{1}-\mu_{2}$

Equal variances are not assumed for this analysis.

Descriptive Statistics: Saline intensity LV

Saline

Treatments

\begin{tabular}{lrrrr} 
LV & N & Mean & StDev & SE Mean \\
\hline NR Sal & 3 & 11797218 & 3392385 & 1958594 \\
Rad Sal & 3 & 18299003 & 11281558 & 6513410
\end{tabular}

Estimation for Difference

\begin{tabular}{cc} 
& $95 \%$ CI for \\
Difference & Difference \\
\hline-6501784 & $(-35766341,22762773)$
\end{tabular}

\section{Test}

Null hypothesis $\quad H_{0}: \mu_{1}-\mu_{2}=0$

Alternative hypothesis $H_{1}: \mu_{1}-\mu_{2} \neq 0$

T-Value DF P-Value 


\begin{tabular}{|l|l|l|l|}
\hline t-test summaries & Aorta & Right Ventricle & Left Ventricle \\
\hline Aortic Outgrowth & 0.530 & NA & NA \\
\hline ROS Assay & 0.772 & NA & NA \\
\hline H and E & 0.760 & 0.041 & 0.562 \\
\hline Thickness & 0.240 & 0.026 & 0.343 \\
\hline Massons Trichrome & 0.777 & 0.469 & 0.996 \\
\hline Immunohistology & 0.600 & 0.920 & 0.440 \\
\hline
\end{tabular}

\section{ANOVAs}

Aortic Outgrowth

General Linear Model: Radiated Outgrowth versus ... iated Treatments

\section{Method}

Factor coding $\quad(-1,0,+1)$

Factor Information

\begin{tabular}{llrl} 
Factor & Type & Levels & Values \\
\hline Radiated Treatments & Fixed & 5 Alb_ccm, ccm, chitocurc, lipids, rad sal
\end{tabular}

Analysis of Variance

\begin{tabular}{lrrrrr} 
Source & DF & Adj SS & Adj MS & F-Value & P-Value \\
\hline Radiated Treatments & 4 & 98948860 & 24737215 & 1.80 & 0.160 \\
Error & 26 & 358205879 & 13777149 & & \\
Total & 30 & 457154739 & & &
\end{tabular}

\section{Model Summary}

\begin{tabular}{rrrr} 
S & R-sq & R-sq(adj) & R-sq(pred) \\
\hline 3711.76 & $21.64 \%$ & $9.59 \%$ & $0.00 \%$
\end{tabular}


Coefficients

\begin{tabular}{lrrrrr} 
Term & Coef & SE Coef & T-Value & P-Value & VIF \\
\hline Constant & 7192 & 668 & 10.77 & 0.000 & \\
Radiated Treatments & & & & & \\
$\quad$ Alb_ccm & 3120 & 1350 & 2.31 & 0.029 & 1.72 \\
ccm & -1423 & 1350 & -1.05 & 0.302 & 1.72 \\
$\quad$ chitocurc & -2118 & 1350 & -1.57 & 0.129 & 1.72 \\
lipids & -37 & 1350 & -0.03 & 0.978 & 1.72
\end{tabular}

\section{Regression Equation}

Radiated

Outgrowth
$=7192+3120$ Radiated Treatments_Alb_ccm

- 1423 Radiated Treatments_ccm

- 2118 Radiated Treatments_chitocurc - 37 Radiated Treatments_lipids

+ 457 Radiated Treatments_rad sal

Fits and Diagnostics for Unusual Observations

\begin{tabular}{|c|c|c|c|c|}
\hline bs & Radiated & Fit & Resid & $\begin{array}{r}\text { Std } \\
\text { Resid }\end{array}$ \\
\hline 4 & 12625 & 5074 & 7551 & $2.23 \mathrm{R}$ \\
\hline
\end{tabular}

\section{Residual Plots for Radiated Outgrowth}

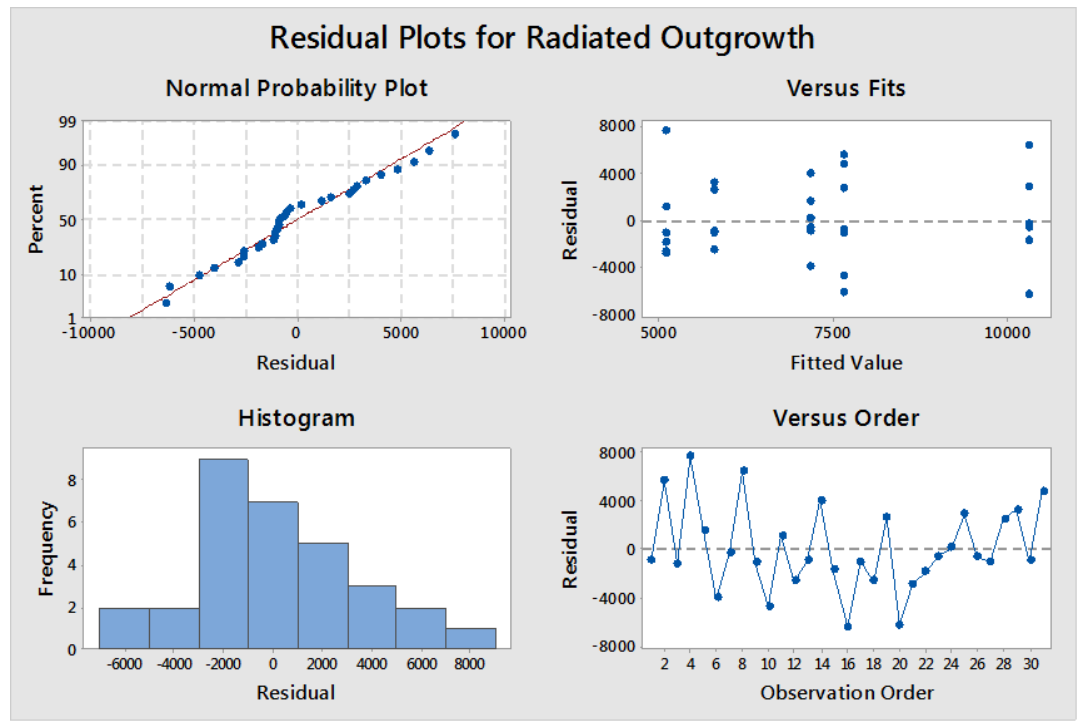

\section{Tukey Test}

\section{Comparisons for Radiated Outgrowth}




\title{
Tukey Pairwise Comparisons: Radiated Treatments
}

\section{Grouping Information Using the Tukey Method and 90\% Confidence}

\author{
Radiated \\ Treatments N Mean Grouping \\ Alb_ccm 610312.5 A

rad sal $7 \quad 7649.3 \mathrm{~A}$ \\ lipids $\quad 6 \quad 7155.0 \mathrm{~A}$ \\ ccm $65769.2 \mathrm{~A}$ \\ chitocurc $6 \quad 5074.1 \mathrm{~A}$ \\ Means that do not share a letter are significantly different.
}

\section{Tukey Simultaneous Tests for Differences of Means}

\begin{tabular}{lrrcrr}
$\begin{array}{l}\text { Difference of Radiated } \\
\text { Treatments Levels }\end{array}$ & $\begin{array}{r}\text { Difference } \\
\text { of Means }\end{array}$ & $\begin{array}{r}\text { SE of } \\
\text { Difference }\end{array}$ & $\begin{array}{c}\text { Simultaneous } \\
90 \% \text { CI }\end{array}$ & $\begin{array}{r}\text { T-Value } \\
\text { T-Value }\end{array}$ \\
\hline ccm - Alb_ccm & -4543 & 2143 & $(-10104,1018)$ & -2.12 & 0.242 \\
chitocurc - Alb_ccm & -5238 & 2143 & $(-10800,323)$ & -2.44 & 0.135 \\
lipids - Alb_ccm & -3157 & 2143 & $(-8719,2404)$ & -1.47 & 0.588 \\
rad sal - Alb_ccm & -2663 & 2065 & $(-8022,2696)$ & -1.29 & 0.700 \\
chitocurc - ccm & -695 & 2143 & $(-6256,4866)$ & -0.32 & 0.997 \\
lipids - ccm & 1386 & 2143 & $(-4175,6947)$ & 0.65 & 0.966 \\
rad sal - ccm & 1880 & 2065 & $(-3479,7239)$ & 0.91 & 0.890 \\
lipids - chitocurc & 2081 & 2143 & $(-3480,7642)$ & 0.97 & 0.866 \\
rad sal - chitocurc & 2575 & 2065 & $(-2784,7934)$ & 1.25 & 0.725 \\
rad sal - lipids & 494 & 2065 & $(-4865,5853)$ & 0.24 & 0.999
\end{tabular}

Individual confidence level $=98.47 \%$

\section{ROS ASSAY}

\section{General Linear Model: Radiated ROS versus Radiated Treatments}

\author{
Method \\ Factor coding $\quad(-1,0,+1)$ \\ Factor Information

\begin{tabular}{llr} 
Factor & Type & Levels Values \\
\hline Radiated Treatments & Fixed & 4 Alb/CCM, Chitocurc, Lipids, Rad Sal
\end{tabular}




\section{Analysis of Variance}

\begin{tabular}{lrrrrr} 
Source & DF & Adj SS & Adj MS & F-Value & P-Value \\
\hline Radiated Treatments & 3 & 0.000318 & 0.000106 & 0.46 & 0.719 \\
Error & 7 & 0.001617 & 0.000231 & & \\
Total & 10 & 0.001935 & & &
\end{tabular}

\section{Model Summary}

\begin{tabular}{rrrr} 
S & R-sq & R-sq(adj) & R-sq(pred) \\
\hline 0.0151971 & $16.45 \%$ & $0.00 \%$ & $0.00 \%$
\end{tabular}

\section{Coefficients}

\begin{tabular}{lrrrrr} 
Term & Coef & SE Coef & T-Value & P-Value & VIF \\
\hline Constant & 0.04460 & 0.00478 & 9.33 & 0.000 & \\
Radiated Treatments & & & & & \\
$\quad$ Alb/CCM & 0.00115 & 0.00719 & 0.16 & 0.878 & 1.55 \\
Chitocurc & -0.01035 & 0.00898 & -1.15 & 0.287 & 1.71 \\
$\quad$ Lipids & 0.00565 & 0.00898 & 0.63 & 0.549 & 1.71
\end{tabular}

\section{Regression Equation}

$$
\begin{array}{ll}
\text { Radiated }= & 0.04460+0.00115 \text { Radiated Treatments_Alb/CCM } \\
\text { ROS } & -0.01035 \text { Radiated Treatments_Chitocurc } \\
& +0.00565 \text { Radiated Treatments_Lipids } \\
& +0.00356 \text { Radiated Treatments_Rad Sal }
\end{array}
$$

Fits and Diagnostics for Unusual Observations

\begin{tabular}{rrrrr}
\multicolumn{3}{c}{ Radiated } & & \multicolumn{2}{c}{$\begin{array}{c}\text { Std } \\
\text { Obs }\end{array}$} \\
ROS & Fit & Resid & Resid \\
\hline 9 & 0.07600 & 0.04575 & 0.03025 & $2.30 \quad R$ \\
$R$ Large residual & & &
\end{tabular}




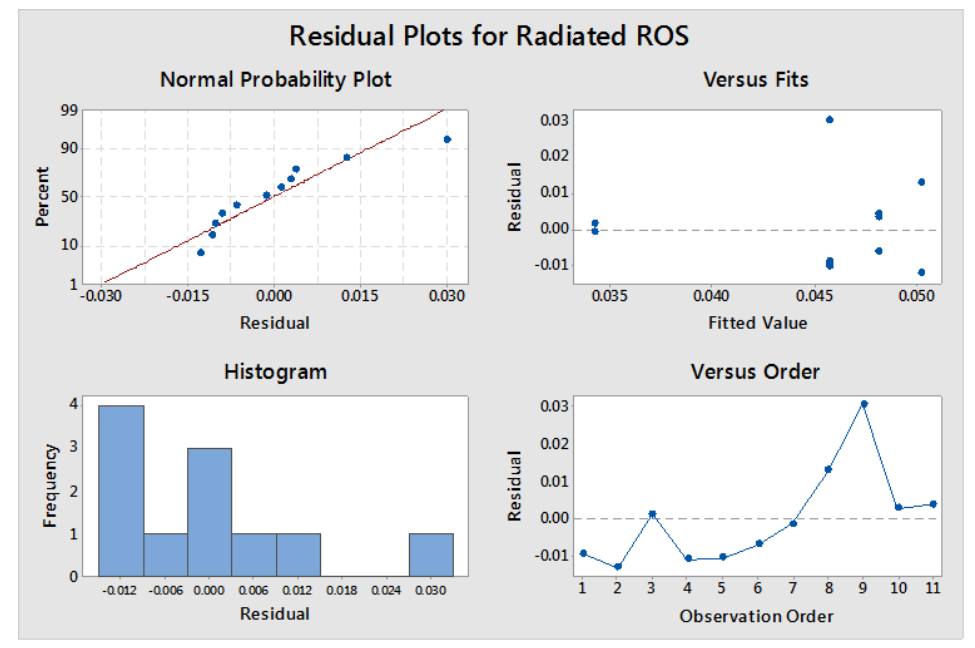

\section{Comparisons for Radiated ROS}

\section{Tukey Pairwise Comparisons: Radiated Treatments}

\section{Grouping Information Using the Tukey Method and 95\% Confidence}

\begin{tabular}{lrr}
$\begin{array}{l}\text { Radiated } \\
\text { Treatments }\end{array}$ & N & Mean Grouping \\
\hline Lipids & 2 & $0.0502500 \mathrm{~A}$ \\
Rad Sal & 3 & $0.0481667 \mathrm{~A}$ \\
Alb/CCM & 4 & $0.0457500 \mathrm{~A}$ \\
Chitocurc & 2 & $0.0342500 \mathrm{~A}$
\end{tabular}

Means that do not share a letter are significantly different.

\section{Tukey Simultaneous Tests for Differences of Means}

\begin{tabular}{|l|r|r|r|r|r|r|}
$\begin{array}{l}\text { Difference of Radiated } \\
\text { Treatments Levels }\end{array}$ & $\begin{array}{r}\text { Difference } \\
\text { of Means }\end{array}$ & $\begin{array}{r}\text { SE of } \\
\text { Difference }\end{array}$ & $\begin{array}{c}\text { Simultaneous } \\
95 \% \text { CI }\end{array}$ & & T-Value & $\begin{array}{r}\text { Adjusted } \\
\text { P-Value }\end{array}$ \\
\hline Chitocurc - Alb/CCM & -0.0115 & 0.0132 & $(-0.0551,0.0321)$ & -0.87 & 0.818 \\
\hline Lipids - Alb/CCM & 0.0045 & 0.0132 & $(-0.0391,0.0481)$ & 0.34 & 0.985 \\
\hline Rad Sal - Alb/CCM & 0.0024 & 0.0116 & $(-0.0360,0.0408)$ & 0.21 & 0.997 \\
\hline Lipids - Chitocurc & 0.0160 & 0.0152 & $(-0.0343,0.0663)$ & 1.05 & 0.726 \\
\hline Rad Sal - Chitocurc & 0.0139 & 0.0139 & $(-0.0320,0.0598)$ & 1.00 & 0.753 \\
\hline Rad Sal - Lipids & -0.0021 & 0.0139 & $(-0.0480,0.0438)$ & -0.15 & 0.999 \\
\hline
\end{tabular}

Individual confidence level $=98.70 \%$

$H \& E$

Aorta 


\title{
General Linear Model: Radiation Muscle Tissue/Nucle_2 ... ents Aorta
}

\author{
Method
}

Factor coding $\quad(-1,0,+1)$

Factor Information

\begin{tabular}{llrl} 
Factor & Type & Levels Values \\
\hline Radiation Treatments Aorta & Fixed & 5 Alb_CCM, CCM, Chitocurc, Lipids, Rad sal
\end{tabular}

Analysis of Variance

\begin{tabular}{lrrrrr} 
Source & DF & Adj SS & Adj MS & F-Value & P-Value \\
\hline Radiation Treatments Aorta & 4 & 6452 & 1613 & 0.99 & 0.454 \\
Error & 10 & 16230 & 1623 & & \\
Total & 14 & 22682 & & &
\end{tabular}

\section{Model Summary}

\begin{tabular}{rrrr} 
S & R-sq & R-sq(adj) & R-sq(pred) \\
\hline 40.2871 & $28.44 \%$ & $0.00 \%$ & $0.00 \%$
\end{tabular}

\section{Coefficients}

\begin{tabular}{lrrrrr} 
Term & Coef & SE Coef & T-Value & P-Value & VIF \\
\hline Constant & 198.7 & 10.7 & 18.64 & 0.000 & \\
Radiation Treatments Aorta & & & & & \\
$\quad$ Alb_CCM & -6.2 & 24.5 & -0.25 & 0.805 & 1.83 \\
CCM & 33.7 & 20.9 & 1.61 & 0.138 & 1.62 \\
Chitocurc & 8.6 & 18.9 & 0.45 & 0.660 & 1.53 \\
Lipids & -29.1 & 20.9 & -1.39 & 0.195 & 1.62
\end{tabular}

\section{Regression Equation}

Radiation Muscle Tissue/Nucle_2 $=198.7$ - 6.2 Radiation Treatments Aorta_Alb_CCM

+ 33.7 Radiation Treatments Aorta_CCM

+ 8.6 Radiation Treatments Aorta_Chitocurc

- 29.1 Radiation Treatments Aorta_Lipids

- 7.0 Radiation Treatments Aorta_Rad sal 


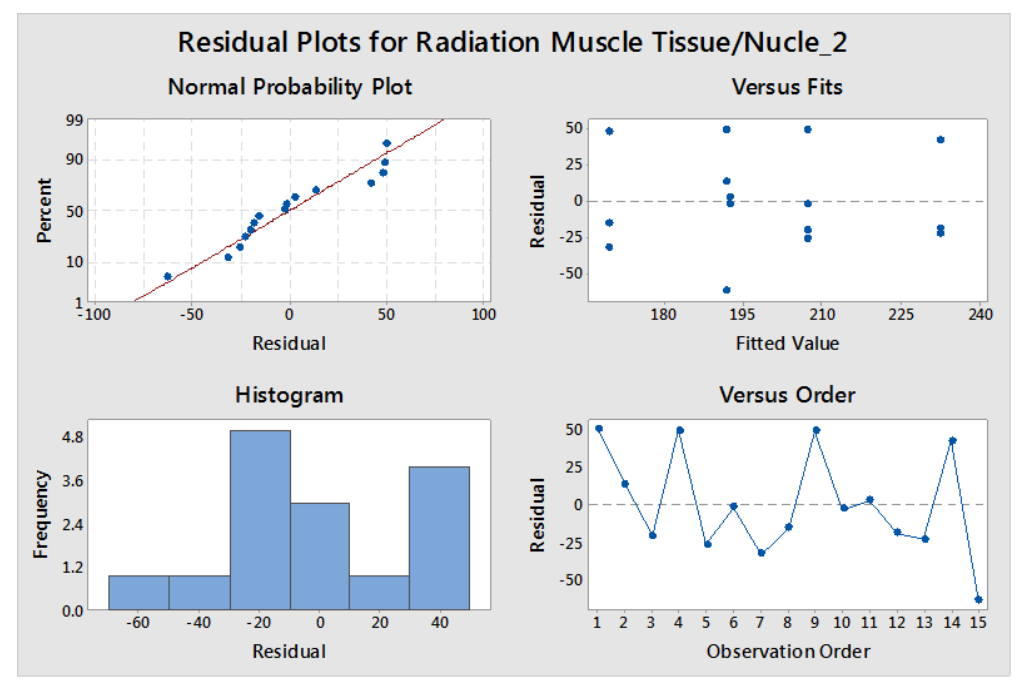

\section{Comparisons for Radiation Muscle Tissue/Nucle_2}

Tukey Pairwise Comparisons: Radiation Treatments Aorta

\section{Grouping Information Using the Tukey Method and 75\% Confidence}

\begin{tabular}{|c|c|c|c|}
\hline $\begin{array}{l}\text { Radiation } \\
\text { Treatments }\end{array}$ & & & \\
\hline Aorta & $\mathrm{N}$ & Mean & Grouping \\
\hline $\mathrm{CCM}$ & 3 & 232.403 & $A$ \\
\hline Chitocurc & 4 & 207.250 & $A$ \\
\hline Alb_CCM & 2 & 192.487 & A \\
\hline Rad sal & 3 & 191.690 & A \\
\hline Lipids & 3 & 169.596 & $A$ \\
\hline
\end{tabular}

Means that do not share a letter are significantly different.

\section{Tukey Simultaneous Tests for Differences of Means}

\begin{tabular}{lrrcrr}
$\begin{array}{l}\text { Difference of Radiation } \\
\text { Treatments Aorta Levels }\end{array}$ & $\begin{array}{r}\text { Difference } \\
\text { of Means }\end{array}$ & $\begin{array}{r}\text { SE of } \\
\text { Difference }\end{array}$ & $\begin{array}{c}\text { Simultaneous } \\
75 \% \text { CI }\end{array}$ & $\begin{array}{r}\text { T-Value } \\
\text { P-Value }\end{array}$ \\
\hline CCM - Alb_CCM & 39.9 & 36.8 & $(-41.5,121.3)$ & 1.09 & 0.810 \\
Chitocurc - Alb_CCM & 14.8 & 34.9 & $(-62.5,92.0)$ & 0.42 & 0.992 \\
Lipids - Alb_CCM & -22.9 & 36.8 & $(-104.3,58.5)$ & -0.62 & 0.968 \\
Rad sal - Alb_CCM & -0.8 & 36.8 & $(-82.2,80.6)$ & -0.02 & 1.000 \\
Chitocurc - CCM & -25.2 & 30.8 & $(-93.3,42.9)$ & -0.82 & 0.919 \\
Lipids - CCM & -62.8 & 32.9 & $(-135.6,10.0)$ & -1.91 & 0.372 \\
Rad sal - CCM & -40.7 & 32.9 & $(-113.5,32.1)$ & -1.24 & 0.732 \\
Lipids - Chitocurc & -37.7 & 30.8 & $(-105.8,30.4)$ & -1.22 & 0.739 \\
Rad sal - Chitocurc & -15.6 & 30.8 & $(-83.7,52.5)$ & -0.51 & 0.985 \\
Rad sal - Lipids & 22.1 & 32.9 & $(-50.7,94.9)$ & 0.67 & 0.958
\end{tabular}


Right Ventricle

\section{General Linear Model: Radiation Muscle Tissue/Nuclei ... reatments RV}

\section{Method}

Factor coding $\quad(-1,0,+1)$

\section{Factor Information}

\begin{tabular}{llrl} 
Factor & Type & Levels Values \\
\hline Radiation Treatments RV & Fixed & 5 Alb/CCM, CCM, Chitocurc, Lipids, Rad Sal
\end{tabular}

\section{Analysis of Variance}

\begin{tabular}{lrrrrr} 
Source & DF & Adj SS & Adj MS & F-Value & P-Value \\
\hline Radiation Treatments RV & 4 & 354.8 & 88.70 & 0.04 & 0.997 \\
Error & 13 & 28970.4 & 2228.49 & & \\
Total & 17 & 29325.2 & & &
\end{tabular}

\section{Model Summary}

\begin{tabular}{rrrr} 
S & R-sq & R-sq(adj) & R-sq(pred) \\
\hline 47.2069 & $1.21 \%$ & $0.00 \%$ & $0.00 \%$
\end{tabular}

\section{Coefficients}

\begin{tabular}{lrrrrr} 
Term & Coef & SE Coef & T-Value & P-Value & VIF \\
\hline Constant & 339.8 & 11.2 & 30.23 & 0.000 & \\
Radiation Treatments RV & & & & & \\
$\quad$ Alb/CCM & 1.4 & 21.5 & 0.07 & 0.948 & 1.65 \\
CCM & 2.3 & 23.9 & 0.10 & 0.925 & 1.78 \\
Chitocurc & 5.0 & 21.5 & 0.23 & 0.818 & 1.65 \\
Lipids & -1.3 & 23.9 & -0.05 & 0.959 & 1.78
\end{tabular}

\section{Regression Equation}

\footnotetext{
Radiation Muscle Tissue/Nuclei $=339.8+1.4$ Radiation Treatments RV_Alb/CCM

+ 2.3 Radiation Treatments RV_CCM

+ 5.0 Radiation Treatments RV_Chitocurc

- 1.3 Radiation Treatments RV_Lipids

- 7.5 Radiation Treatments RV_Rad Sal
} 


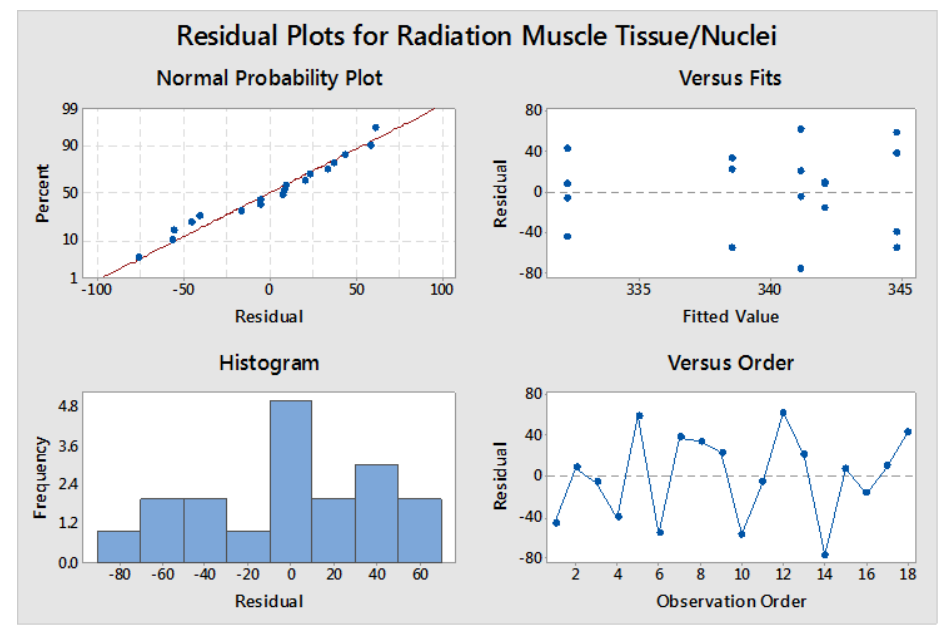

\section{Comparisons for Radiation Muscle Tissue/Nuclei}

Tukey Pairwise Comparisons: Radiation Treatments RV

\section{Grouping Information Using the Tukey Method and 75\% Confidence}

\begin{tabular}{|c|c|c|c|}
\hline Radiation & & & \\
\hline Treatments RV & $\mathrm{N}$ & Mean & Grouping \\
\hline Chitocurc & 4 & 344.798 & A \\
\hline $\mathrm{CCM}$ & 3 & 342.053 & A \\
\hline Alb/CCM & 4 & 341.175 & A \\
\hline Lipids & 3 & 338.509 & A \\
\hline Rad Sal & 4 & 332.263 & A \\
\hline
\end{tabular}

Means that do not share a letter are significantly different.

\section{Tukey Simultaneous Tests for Differences of Means}

\begin{tabular}{lrrcrr}
$\begin{array}{l}\text { Difference of Radiation } \\
\text { Treatments RV Levels }\end{array}$ & $\begin{array}{r}\text { Difference } \\
\text { of Means }\end{array}$ & $\begin{array}{r}\text { SE of } \\
\text { Difference }\end{array}$ & $\begin{array}{c}\text { Simultaneous } \\
75 \% \text { CI }\end{array}$ & $\begin{array}{r}\text { T-Value } \\
\text { P-Value }\end{array}$ \\
\hline CCM - Alb/CCM & 0.9 & 36.1 & $(-77.4,79.1)$ & 0.02 & 1.000 \\
Chitocurc - Alb/CCM & 3.6 & 33.4 & $(-68.8,76.1)$ & 0.11 & 1.000 \\
Lipids - Alb/CCM & -2.7 & 36.1 & $(-80.9,75.6)$ & -0.07 & 1.000 \\
Rad Sal - Alb/CCM & -8.9 & 33.4 & $(-81.4,63.6)$ & -0.27 & 0.999 \\
Chitocurc - CCM & 2.7 & 36.1 & $(-75.5,81.0)$ & 0.08 & 1.000 \\
Lipids - CCM & -3.5 & 38.5 & $(-87.2,80.1)$ & -0.09 & 1.000 \\
Rad Sal - CCM & -9.8 & 36.1 & $(-88.1,68.5)$ & -0.27 & 0.999 \\
Lipids - Chitocurc & -6.3 & 36.1 & $(-84.6,72.0)$ & -0.17 & 1.000 \\
Rad Sal - Chitocurc & -12.5 & 33.4 & $(-85.0,59.9)$ & -0.38 & 0.995 \\
Rad Sal - Lipids & -6.2 & 36.1 & $(-84.5,72.0)$ & -0.17 & 1.000
\end{tabular}

Individual confidence level $=95.09 \%$ 
Left Ventricle

\title{
General Linear Model: Radiation Muscle Tissue/Nucle_1 ... atments LV
}

\author{
Method \\ Factor coding $\quad(-1,0,+1)$
}

\section{Factor Information}

\begin{tabular}{llrl} 
Factor & Type & Levels & Values \\
\hline Radiation Treatments LV & Fixed & 5 Alb/CCM, CCM, Chitocurc, Lipids, Rad Sal
\end{tabular}

\section{Analysis of Variance}

\begin{tabular}{lrrrrr} 
Source & DF & Adj SS & Adj MS & F-Value & P-Value \\
\hline Radiation Treatments LV & 4 & 6266 & 1566 & 1.22 & 0.347 \\
Error & 14 & 18012 & 1287 & & \\
Total & 18 & 24278 & & &
\end{tabular}

\section{Model Summary}

\begin{tabular}{rrrr} 
S & R-sq & R-sq(adj) & R-sq(pred) \\
\hline 35.8692 & $25.81 \%$ & $4.61 \%$ & $0.00 \%$
\end{tabular}

\section{Coefficients}

\begin{tabular}{lrrrrr} 
Term & Coef & SE Coef & T-Value & P-Value & VIF \\
\hline Constant & 306.65 & 8.28 & 37.02 & 0.000 & \\
Radiation Treatments LV & & & & & \\
$\quad$ Alb/CCM & -7.6 & 16.2 & -0.47 & 0.644 & 1.63 \\
CCM & 35.4 & 16.2 & 2.19 & 0.046 & 1.63 \\
Chitocurc & -10.4 & 16.2 & -0.64 & 0.532 & 1.63 \\
$\quad$ Lipids & -13.5 & 18.1 & -0.75 & 0.467 & 1.76
\end{tabular}

\section{Regression Equation}

Radiation Muscle Tissue/Nucle_1 $=306.65-7.6$ Radiation Treatments LV_Alb/CCM

+ 35.4 Radiation Treatments LV_CCM

- 10.4 Radiation Treatments LV_Chitocurc

- 13.5 Radiation Treatments LV_Lipids

- 3.9 Radiation Treatments LV_Rad Sal

Fits and Diagnostics for Unusual Observations 


\begin{tabular}{|c|c|c|c|c|}
\hline \multicolumn{4}{|c|}{ Radiation Muscle } & \multirow{2}{*}{$\begin{array}{r}\text { Std } \\
\text { Resid }\end{array}$} \\
\hline Obs & Tissue/Nucle_1 & Fit & Resid & \\
\hline 5 & 364.2 & 296.3 & 67.9 & $2.19 \mathrm{R}$ \\
\hline 19 & 375.8 & 302.8 & 73.0 & $2.35 \mathrm{R}$ \\
\hline
\end{tabular}

$R$ Large residual

Residual Plots for Radiation Muscle Tissue/Nucle_1

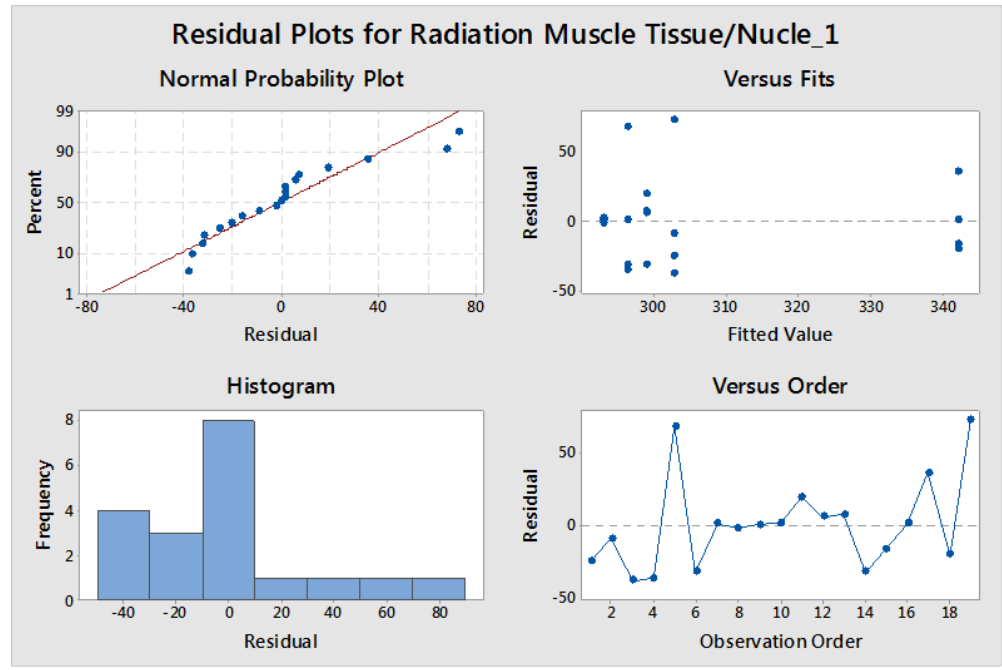

\section{Comparisons for Radiation Muscle Tissue/Nucle_1}

Tukey Pairwise Comparisons: Radiation Treatments LV

Grouping Information Using the Tukey Method and 75\% Confidence

\begin{tabular}{|c|c|c|c|}
\hline Radiation & $N$ & Mean & Grouning \\
\hline CCM & 4 & 342.024 & A \\
\hline Rad Sal & 4 & 302.798 & A \\
\hline Alb/CCM & 4 & 299.012 & A \\
\hline Chitocurc & 4 & 296.286 & A \\
\hline Lipids & 3 & 293.140 & A \\
\hline
\end{tabular}

Means that do not share a letter are significantly different.

\section{Tukey Simultaneous Tests for Differences of Means}

$\begin{array}{lrrrrr}\begin{array}{l}\text { Difference of Radiation } \\ \text { Treatments LV Levels }\end{array} & \begin{array}{r}\text { Difference } \\ \text { of Means }\end{array} & \begin{array}{r}\text { SE of } \\ \text { Difference }\end{array} & \begin{array}{c}\text { Simultaneous } \\ 75 \% \text { CI }\end{array} & \begin{array}{r}\text { Adjusted } \\ \text { T-Value }\end{array} & \begin{array}{r}\text { P-Value } \\ \hline \text { CCM - Alb/CCM }\end{array} \\ \text { Chitocurc - Alb/CCM } & 43.0 & 25.4 & (-11.7,97.7) & 1.70 & 0.467 \\ & -2.7 & 25.4 & (-57.4,52.0) & -0.11 & 1.000\end{array}$




$\begin{array}{lrrrrr}\text { Lipids - Alb/CCM } & -5.9 & 27.4 & (-65.0,53.2) & -0.21 & 0.999 \\ \text { Rad Sal - Alb/CCM } & 3.8 & 25.4 & (-50.9,58.5) & 0.15 & 1.000 \\ \text { Chitocurc - CCM } & -45.7 & 25.4 & (-100.4,9.0) & -1.80 & 0.409 \\ \text { Lipids - CCM } & -48.9 & 27.4 & (-108.0,10.2) & -1.78 & 0.419 \\ \text { Rad Sal - CCM } & -39.2 & 25.4 & (-93.9,15.5) & -1.55 & 0.552 \\ \text { Lipids - Chitocurc } & -3.1 & 27.4 & (-62.2,55.9) & -0.11 & 1.000 \\ \text { Rad Sal - Chitocurc } & 6.5 & 25.4 & (-48.2,61.2) & 0.26 & 0.999 \\ \text { Rad Sal - Lipids } & 9.7 & 27.4 & (-49.4,68.7) & 0.35 & 0.996\end{array}$

Individual confidence level $=95.11 \%$

Thickness Measurements

Aorta

\title{
General Linear Model: Radiation Thickness Aorta versus ... ents Aorta
}

\author{
Method \\ Factor coding $\quad(-1,0,+1)$
}

\section{Factor Information}

\begin{tabular}{llrl} 
Factor & Type & Levels Values \\
\hline Radiation Treatments Aorta & Fixed & 5 Alb CCM, CCM, chitocurc, Lipids, Rad Sal
\end{tabular}

\section{Analysis of Variance}

\begin{tabular}{lrrrrr} 
Source & DF & Adj SS & Adj MS & F-Value & P-Value \\
\hline Radiation Treatments Aorta & 4 & 2374 & 593.4 & 0.90 & 0.501 \\
Error & 10 & 6622 & 662.2 & & \\
Total & 14 & 8995 & & &
\end{tabular}

\section{Model Summary}

\begin{tabular}{rrrr} 
S & R-sq & R-sq(adj) & R-sq(pred) \\
\hline 25.7323 & $26.39 \%$ & $0.00 \%$ & $0.00 \%$
\end{tabular}

\section{Coefficients}

\begin{tabular}{lrrrrr} 
Term & Coef & SE Coef & T-Value & P-Value & VIF \\
\hline Constant & 71.06 & 6.81 & 10.44 & 0.000 & \\
Radiation Treatments Aorta & & & & & \\
$\quad$ Alb CCM & -20.7 & 15.7 & -1.33 & 0.214 & 1.83 \\
CCM & 16.1 & 13.4 & 1.20 & 0.257 & 1.62 \\
chitocurc & 5.0 & 12.1 & 0.41 & 0.690 & 1.53
\end{tabular}


Lipids

\section{Regression Equation}

$$
\begin{aligned}
\text { Radiation Thickness Aorta }= & 71.06-20.7 \text { Radiation Treatments Aorta_Alb CCM } \\
& +16.1 \text { Radiation Treatments Aorta_CCM } \\
& +5.0 \text { Radiation Treatments Aorta_chitocurc } \\
& -10.8 \text { Radiation Treatments Aorta_Lipids } \\
& +10.5 \text { Radiation Treatments Aorta_Rad Sal }
\end{aligned}
$$

\section{Fits and Diagnostics for Unusual Observations}

\begin{tabular}{|c|c|c|c|}
\hline $\begin{array}{l}\text { Radiation } \\
\text { Treatments }\end{array}$ & & & \\
\hline Aorta & $\mathrm{N}$ & Mean & Grouping \\
\hline $\mathrm{CCM}$ & 3 & 87.1434 & $A$ \\
\hline Rad Sal & 3 & 81.5788 & $A$ \\
\hline chitocurc & 4 & 76.0221 & $A$ \\
\hline Lipids & 3 & 60.2502 & $A$ \\
\hline Alb CCM & 2 & 50.3127 & $A$ \\
\hline
\end{tabular}

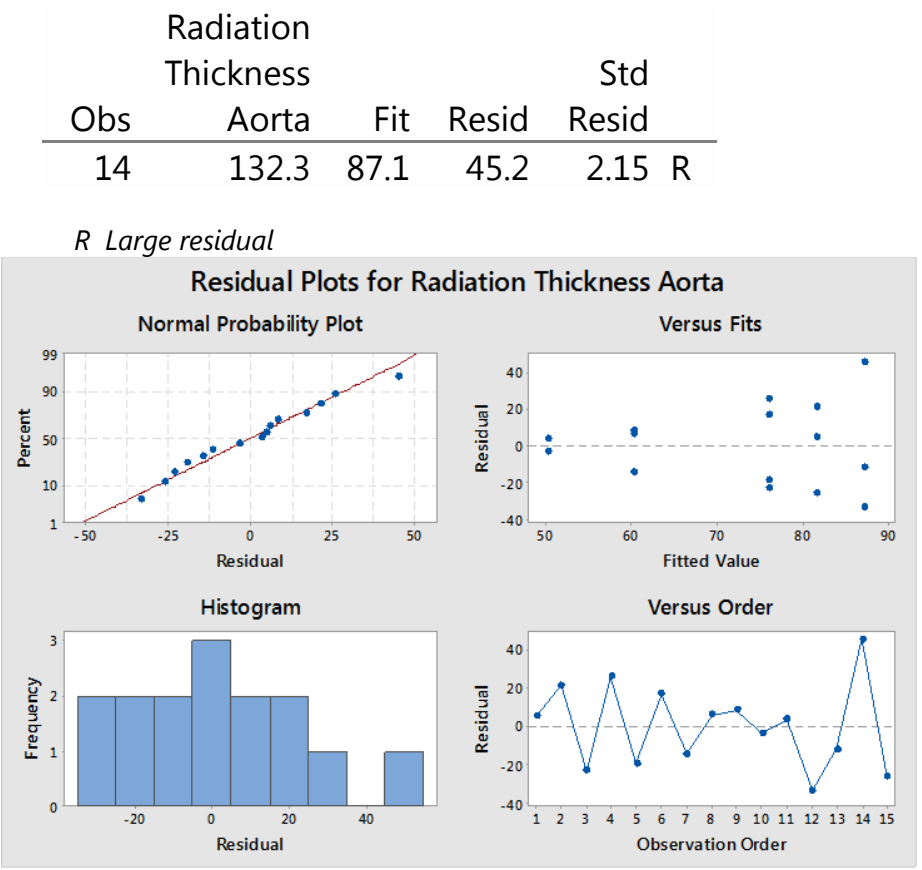

Comparisons for Radiation Thickness Aorta

Tukey Pairwise Comparisons: Radiation Treatments Aorta

Grouping Information Using the Tukey Method and 80\% Confidence 
Means that do not share a letter are significantly different.

Tukey Simultaneous Tests for Differences of Means

\begin{tabular}{lrrcrr}
$\begin{array}{l}\text { Difference of Radiation } \\
\text { Treatments Aorta Levels }\end{array}$ & $\begin{array}{r}\text { Difference } \\
\text { of Means }\end{array}$ & $\begin{array}{r}\text { SE of } \\
\text { Difference }\end{array}$ & $\begin{array}{c}\text { Simultaneous } \\
80 \% \text { CI }\end{array}$ & $\begin{array}{r}\text { T-Value } \\
\text { P-Value }\end{array}$ \\
\hline CCM - Alb CCM & 36.8 & 23.5 & $(-19.0,92.6)$ & 1.57 & 0.547 \\
chitocurc - Alb CCM & 25.7 & 22.3 & $(-27.2,78.7)$ & 1.15 & 0.776 \\
Lipids - Alb CCM & 9.9 & 23.5 & $(-45.9,65.7)$ & 0.42 & 0.992 \\
Rad Sal - Alb CCM & 31.3 & 23.5 & $(-24.5,87.1)$ & 1.33 & 0.680 \\
chitocurc - CCM & -11.1 & 19.7 & $(-57.8,35.6)$ & -0.57 & 0.977 \\
Lipids - CCM & -26.9 & 21.0 & $(-76.8,23.0)$ & -1.28 & 0.708 \\
Rad Sal - CCM & -5.6 & 21.0 & $(-55.5,44.4)$ & -0.26 & 0.999 \\
Lipids - chitocurc & -15.8 & 19.7 & $(-62.5,30.9)$ & -0.80 & 0.924 \\
Rad Sal - chitocurc & 5.6 & 19.7 & $(-41.1,52.3)$ & 0.28 & 0.998 \\
Rad Sal - Lipids & 21.3 & 21.0 & $(-28.6,71.2)$ & 1.02 & 0.843
\end{tabular}

Individual confidence level $=96.11 \%$

Right Ventricle

\section{General Linear Model: Radiation Thickness RV versus ... reatments $\mathrm{RV}$}

\section{Method}

Factor coding $\quad(-1,0,+1)$

\section{Factor Information}

\begin{tabular}{llrl} 
Factor & Type & Levels & Values \\
\hline Radiation Treatments RV & Fixed & 5 Alb CCM, CCM, Chitocurc, Lipids, Rad Sal
\end{tabular}

\section{Analysis of Variance}

\begin{tabular}{lrrrrr} 
Source & DF & Adj SS & Adj MS & F-Value & P-Value \\
\hline Radiation Treatments RV & 4 & 50902 & 12725 & 0.89 & 0.497 \\
Error & 14 & 200696 & 14335 & & \\
Total & 18 & 251598 & & &
\end{tabular}

\section{Model Summary}

\begin{tabular}{rrrr} 
S & R-sq & R-sq(adj) & R-sq(pred) \\
\hline 119.731 & $20.23 \%$ & $0.00 \%$ & $0.00 \%$
\end{tabular}


Coefficients

\begin{tabular}{lrrrrr} 
Term & Coef & SE Coef & T-Value & P-Value & VIF \\
\hline Constant & 905.8 & 27.7 & 32.76 & 0.000 & \\
Radiation Treatments RV & & & & & \\
$\quad$ Alb CCM & 13.0 & 54.0 & 0.24 & 0.814 & 1.63 \\
CCM & -25.9 & 54.0 & -0.48 & 0.639 & 1.63 \\
Chitocurc & -47.5 & 54.0 & -0.88 & 0.394 & 1.63 \\
Lipids & -33.5 & 60.3 & -0.56 & 0.587 & 1.76
\end{tabular}

\section{Regression Equation}

$$
\begin{aligned}
\text { Radiation Thickness RV }= & 905.8+13.0 \text { Radiation Treatments RV_Alb CCM } \\
& -25.9 \text { Radiation Treatments RV_CCM } \\
& -47.5 \text { Radiation Treatments RV_Chitocurc } \\
& -33.5 \text { Radiation Treatments RV_Lipids } \\
& +93.9 \text { Radiation Treatments RV_Rad Sal }
\end{aligned}
$$

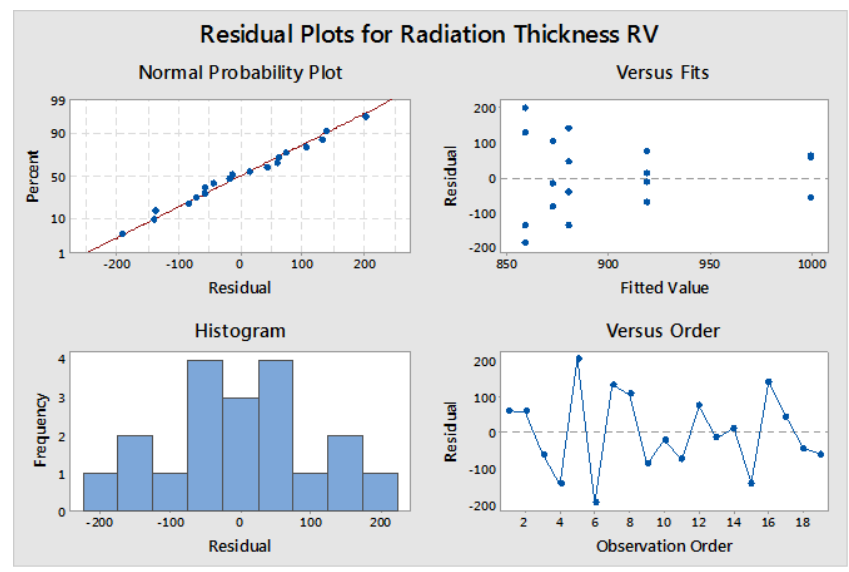

\section{Comparisons for Radiation Thickness RV}

\section{Tukey Pairwise Comparisons: Radiation Treatments RV}

Grouping Information Using the Tukey Method and 80\% Confidence

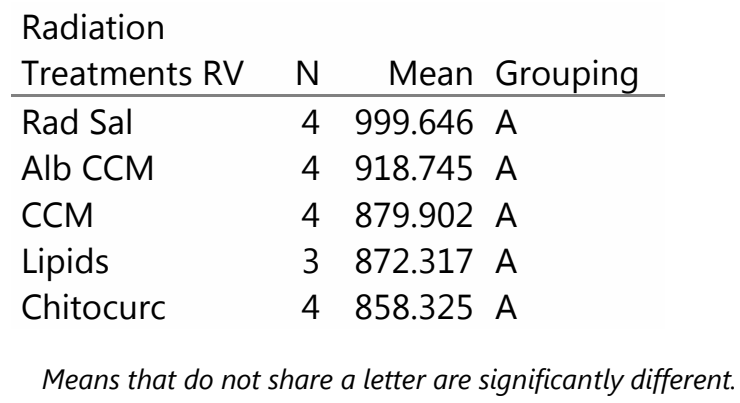




\section{Tukey Simultaneous Tests for Differences of Means}

\begin{tabular}{lrrcrr}
$\begin{array}{l}\text { Difference of Radiation } \\
\text { Treatments RV Levels }\end{array}$ & $\begin{array}{r}\text { Difference } \\
\text { of Means }\end{array}$ & $\begin{array}{r}\text { SE of } \\
\text { Difference }\end{array}$ & $\begin{array}{c}\text { Simultaneous } \\
80 \% \text { CI }\end{array}$ & $\begin{array}{r}\text { T-Value } \\
\text { P-Value }\end{array}$ \\
\hline CCM - Alb CCM & -38.8 & 84.7 & $(-234.0,156.3)$ & -0.46 & 0.990 \\
Chitocurc - Alb CCM & -60.4 & 84.7 & $(-255.6,134.7)$ & -0.71 & 0.950 \\
Lipids - Alb CCM & -46.4 & 91.4 & $(-257.2,164.4)$ & -0.51 & 0.985 \\
Rad Sal - Alb CCM & 80.9 & 84.7 & $(-114.3,276.1)$ & 0.96 & 0.870 \\
Chitocurc - CCM & -21.6 & 84.7 & $(-216.7,173.6)$ & -0.25 & 0.999 \\
Lipids - CCM & -7.6 & 91.4 & $(-218.4,203.2)$ & -0.08 & 1.000 \\
Rad Sal - CCM & 119.7 & 84.7 & $(-75.4,314.9)$ & 1.41 & 0.629 \\
Lipids - Chitocurc & 14.0 & 91.4 & $(-196.8,224.8)$ & 0.15 & 1.000 \\
Rad Sal - Chitocurc & 141.3 & 84.7 & $(-53.8,336.5)$ & 1.67 & 0.482 \\
Rad Sal - Lipids & 127.3 & 91.4 & $(-83.5,338.1)$ & 1.39 & 0.642
\end{tabular}

Individual confidence level $=96.30 \%$

Left Ventricle

\section{General Linear Model: Radiation Thickness LV versus ... Treatments LV}

\section{Method}

Factor coding $\quad(-1,0,+1)$

Factor Information

\begin{tabular}{llrl} 
Factor & Type & Levels & Values \\
\hline Radiation Treatments LV & Fixed & 5 Alb CCM, CCM, Chitocurc, Lipids, Rad Sal
\end{tabular}

\section{Analysis of Variance}

\begin{tabular}{lrrrrr} 
Source & DF & Adj SS & Adj MS & F-Value & P-Value \\
\hline Radiation Treatments LV & 4 & 3779759 & 944940 & 11.62 & 0.000 \\
Error & 14 & 1138661 & 81333 & & \\
Total & 18 & 4918420 & & &
\end{tabular}

\section{Model Summary}

\begin{tabular}{rrrr} 
S & R-sq & R-sq(adj) & R-sq(pred) \\
\hline 285.189 & $76.85 \%$ & $70.23 \%$ & $58.70 \%$
\end{tabular}

\section{Coefficients}




\begin{tabular}{lrrrrr} 
Term & Coef & SE Coef & T-Value & P-Value & VIF \\
\hline Constant & 1574.2 & 65.9 & 23.90 & 0.000 & \\
Radiation Treatments LV & & & & & \\
$\quad$ Alb CCM & 496 & 129 & 3.86 & 0.002 & 1.63 \\
CCM & 576 & 129 & 4.48 & 0.001 & 1.63 \\
Chitocurc & -445 & 129 & -3.46 & 0.004 & 1.63 \\
Lipids & -387 & 144 & -2.69 & 0.017 & 1.76
\end{tabular}

\section{Regression Equation}

Radiation Thickness LV $=1574.2+496$ Radiation Treatments LV_Alb CCM

+ 576 Radiation Treatments LV_CCM

- 445 Radiation Treatments LV_Chitocurc

- 387 Radiation Treatments LV_Lipids

- 241 Radiation Treatments LV_Rad Sal

Fits and Diagnostics for Unusual Observations

\begin{tabular}{|c|c|c|c|c|}
\hline \multicolumn{5}{|c|}{$\begin{array}{l}\text { Radiation } \\
\text { Thickness }\end{array}$} \\
\hline Obs & LV & Fit & Resid & Std Resid \\
\hline 2 & 805 & 1333 & -528 & $-2.14 R$ \\
\hline 19 & 2074 & 1333 & 741 & $3.00 R$ \\
\hline
\end{tabular}

$R$ Large residual

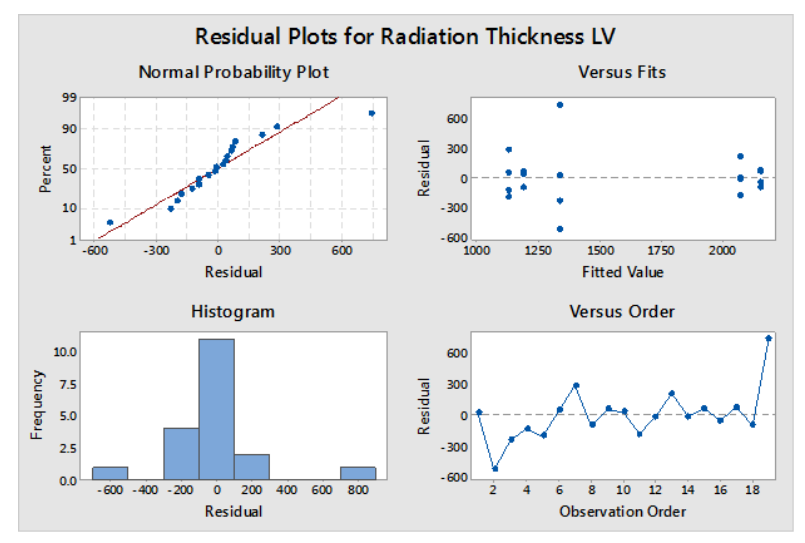

\section{Comparisons for Radiation Thickness LV}

\section{Tukey Pairwise Comparisons: Radiation Treatments LV}

Grouping Information Using the Tukey Method and 80\% Confidence

Radiation

Treatments LV N Mean Grouping 


\begin{tabular}{|c|c|c|}
\hline CCM & 4 & $2150.54 A$ \\
\hline Alb CCM & 4 & $2070.66 \mathrm{~A}$ \\
\hline Rad Sal & 4 & 1333.33 \\
\hline Lipids & 3 & 1187.62 \\
\hline Chitocurc & 4 & 1129.04 \\
\hline
\end{tabular}

Means that do not share a letter are significantly different.

Tukey Simultaneous Tests for Differences of Means

\begin{tabular}{lrrcrr}
$\begin{array}{l}\text { Difference of Radiation } \\
\text { Treatments LV Levels }\end{array}$ & $\begin{array}{r}\text { Difference } \\
\text { of Means }\end{array}$ & $\begin{array}{r}\text { SE of } \\
\text { Difference }\end{array}$ & $\begin{array}{c}\text { Simultaneous } \\
80 \% \text { CI }\end{array}$ & $\begin{array}{r}\text { T-Value } \\
\text { P-Value }\end{array}$ \\
\hline CCM - Alb CCM & 80 & 202 & $(-385,545)$ & 0.40 & 0.994 \\
Chitocurc - Alb CCM & -942 & 202 & $(-1406,-477)$ & -4.67 & 0.003 \\
Lipids - Alb CCM & -883 & 218 & $(-1385,-381)$ & -4.05 & 0.009 \\
Rad Sal - Alb CCM & -737 & 202 & $(-1202,-272)$ & -3.66 & 0.018 \\
Chitocurc - CCM & -1022 & 202 & $(-1486,-557)$ & -5.07 & 0.001 \\
Lipids - CCM & -963 & 218 & $(-1465,-461)$ & -4.42 & 0.004 \\
Rad Sal - CCM & -817 & 202 & $(-1282,-352)$ & -4.05 & 0.009 \\
Lipids - Chitocurc & 59 & 218 & $(-444,561)$ & 0.27 & 0.999 \\
Rad Sal - Chitocurc & 204 & 202 & $(-261,669)$ & 1.01 & 0.845 \\
Rad Sal - Lipids & 146 & 218 & $(-356,648)$ & 0.67 & 0.960
\end{tabular}

Individual confidence level $=96.30 \%$

Masson Trichrome

Aorta

\section{General Linear Model: Adventitia Thickness CCM versus ... ckness CCM}

\section{Method}

Factor coding $\quad(-1,0,+1)$

\section{Factor Information}

Factor Type Levels Values

Treatment Aorta Thickness CCM Fixed 5 Alb_CCM, CCM, Chitocurc, Lipids, Rad Sal

\section{Analysis of Variance}

\begin{tabular}{lrrrrr} 
Source & DF & Adj SS & Adj MS & F-Value & P-Value \\
\hline Treatment Aorta Thickness CCM & 4 & 100.6 & 25.14 & 0.42 & 0.788
\end{tabular}


Error

$9 \quad 534.5 \quad 59.39$

Total

$13 \quad 635.1$

Model Summary

\begin{tabular}{rrrr} 
S & R-sq & R-sq(adj) & R-sq(pred) \\
\hline 7.70652 & $15.83 \%$ & $0.00 \%$ & $0.00 \%$
\end{tabular}

\section{Coefficients}

\begin{tabular}{lrrrrr} 
Term & Coef & SE Coef & T-Value & P-Value & VIF \\
\hline Constant & 15.01 & 2.09 & 7.19 & 0.000 & \\
Treatment Aorta Thickness CCM & & & & & \\
Alb_CCM & -3.96 & 4.71 & -0.84 & 0.422 & 1.84 \\
CCM & 4.08 & 4.03 & 1.01 & 0.338 & 1.64 \\
Chitocurc & 0.71 & 4.03 & 0.18 & 0.863 & 1.64 \\
Lipids & -2.14 & 4.03 & -0.53 & 0.608 & 1.64
\end{tabular}

\section{Regression Equation}

Adventitia Thickness CCM $=15.01-3.96$ Treatment Aorta Thickness CCM_Alb_CCM

+ 4.08 Treatment Aorta Thickness CCM_CCM

+ 0.71 Treatment Aorta Thickness CCM_Chitocurc

- 2.14 Treatment Aorta Thickness CCM_Lipids

+ 1.31 Treatment Aorta Thickness CCM_Rad Sal

Residual Plots for Adventitia Thickness CCM

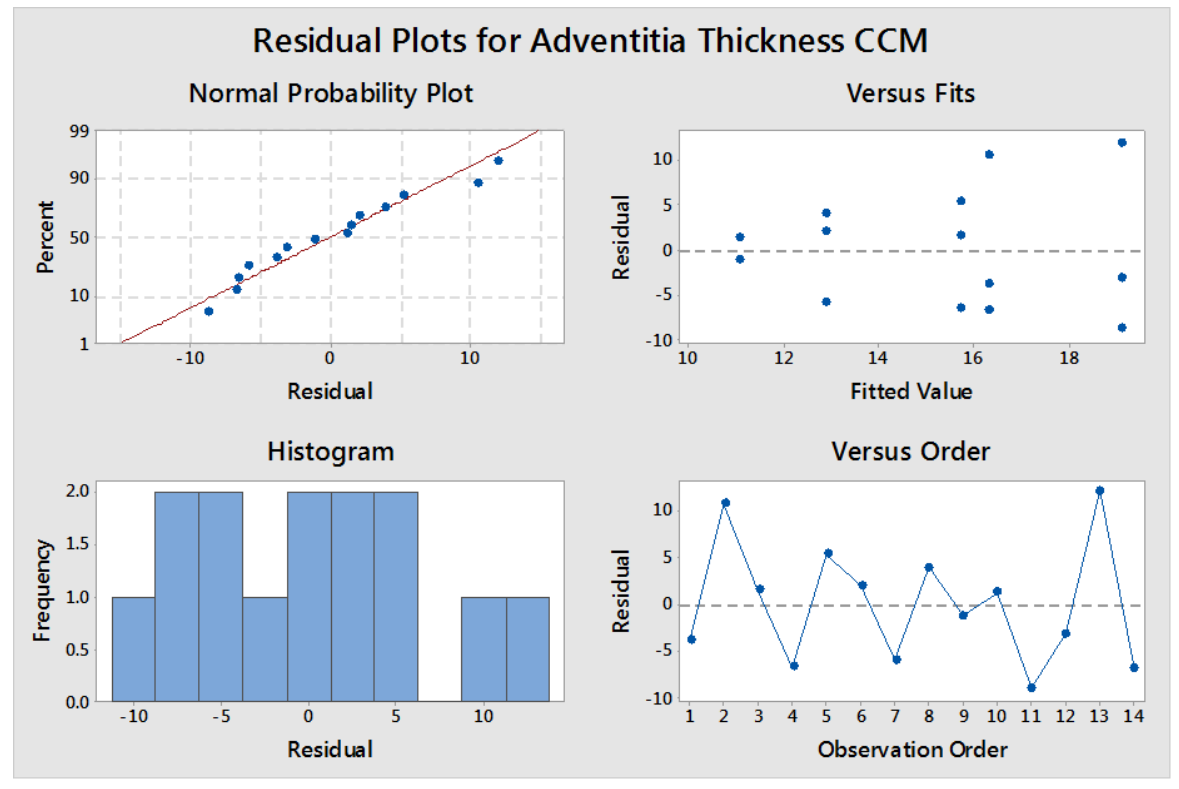




\title{
General Linear Model: \%Adventitia of total thicknes_2 ... ickness CCM
}

\author{
Method
}

Factor coding $\quad(-1,0,+1)$

Factor Information

\begin{tabular}{llrl} 
Factor & Type & Levels Values \\
\hline Treatment Aorta Thickness CCM & Fixed & 5 Alb_CCM, CCM, Chitocurc, Lipids, Rad Sal
\end{tabular}

Analysis of Variance

\begin{tabular}{lrrrrr} 
Source & DF & Adj SS & Adj MS & F-Value & P-Value \\
\hline Treatment Aorta Thickness CCM & 4 & 67.70 & 16.93 & 0.38 & 0.819 \\
Error & 9 & 402.66 & 44.74 & & \\
Total & 13 & 470.36 & & &
\end{tabular}

\section{Model Summary}

\begin{tabular}{rrrr} 
S & R-sq & R-sq(adj) & R-sq(pred) \\
\hline 6.68883 & $14.39 \%$ & $0.00 \%$ & $0.00 \%$
\end{tabular}

\section{Coefficients}

\begin{tabular}{lrrrrr} 
Term & Coef & SE Coef & T-Value & P-Value & VIF \\
\hline Constant & 19.71 & 1.81 & 10.88 & 0.000 & \\
Treatment Aorta Thickness CCM & & & & & \\
Alb_CCM & -2.58 & 4.09 & -0.63 & 0.544 & 1.84 \\
CCM & 3.80 & 3.50 & 1.09 & 0.306 & 1.64 \\
Chitocurc & 0.89 & 3.50 & 0.25 & 0.806 & 1.64 \\
Lipids & -0.40 & 3.50 & -0.11 & 0.912 & 1.64
\end{tabular}

\section{Regression Equation}

\%Adventitia of total thicknes_2 $=19.71-2.58$ Treatment Aorta Thickness CCM_Alb_CCM + 3.80 Treatment Aorta Thickness CCM_CCM

+ 0.89 Treatment Aorta Thickness CCM_Chitocurc

- 0.40 Treatment Aorta Thickness CCM_Lipids

- 1.71 Treatment Aorta Thickness CCM_Rad Sal

Residual Plots for \%Adventitia of total thicknes_2 


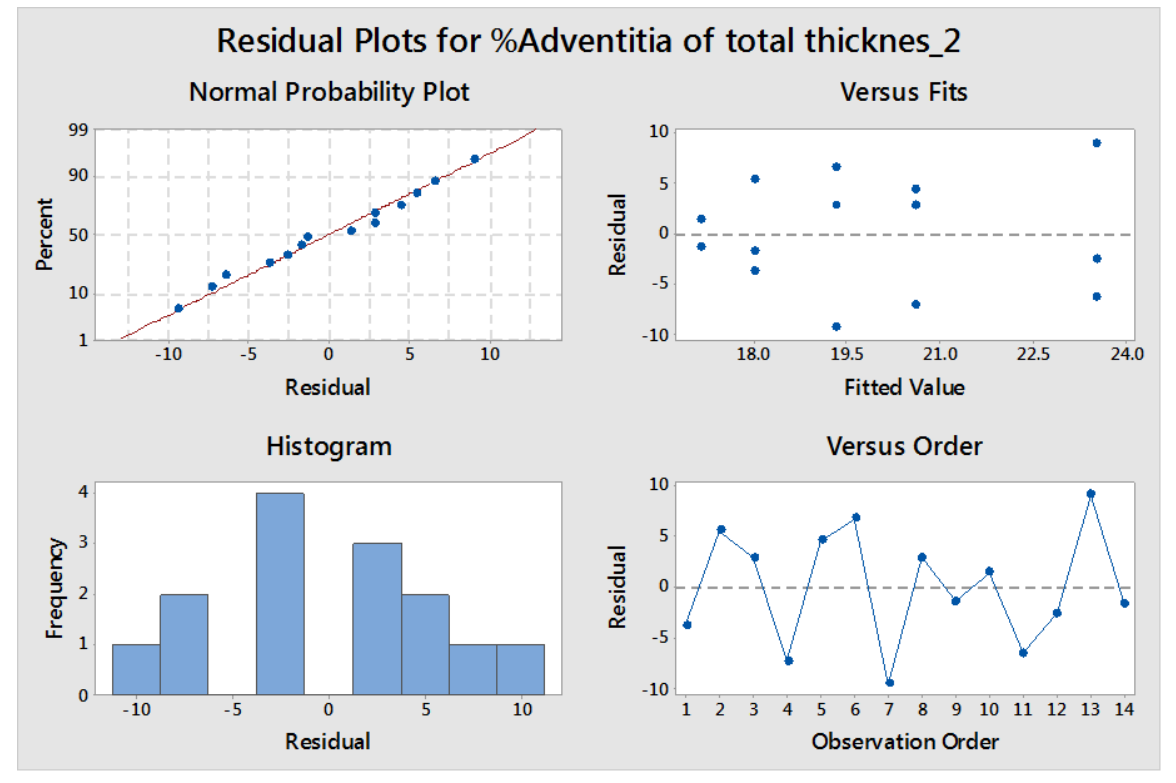

\section{General Linear Model: Radiation \% collagen Aorta versus ... ents Aorta}

\section{Method}

Factor coding $\quad(-1,0,+1)$

\section{Factor Information}

\begin{tabular}{llrl} 
Factor & Type & Levels Values \\
\hline Radiation Treatments Aorta & Fixed & 5 Alb_CCM, CCM, Chitocurc, Lipids, Rad Sal
\end{tabular}

\section{Analysis of Variance}

\begin{tabular}{lrrrrr} 
Source & DF & Adj SS & Adj MS & F-Value & P-Value \\
\hline Radiation Treatments Aorta & 4 & 37.36 & 9.340 & 0.35 & 0.840 \\
Error & 9 & 241.97 & 26.885 & & \\
Total & 13 & 279.33 & & &
\end{tabular}

\section{Model Summary}

\begin{tabular}{rrrr} 
S & R-sq & R-sq(adj) & R-sq(pred) \\
\hline 5.18511 & $13.37 \%$ & $0.00 \%$ & $0.00 \%$
\end{tabular}

\section{Coefficients}

\begin{tabular}{lrrrrr} 
Term & Coef & SE Coef & T-Value & P-Value & VIF \\
\hline Constant & 10.08 & 1.40 & 7.18 & 0.000 &
\end{tabular}


Radiation Treatments Aorta

$\begin{array}{lrrrrr}\text { Alb_CCM } & 0.79 & 3.17 & 0.25 & 0.809 & 1.84 \\ \text { CCM } & -3.08 & 2.71 & -1.14 & 0.285 & 1.64 \\ \text { Chitocurc } & 1.41 & 2.71 & 0.52 & 0.616 & 1.64 \\ \text { Lipids } & 0.13 & 2.71 & 0.05 & 0.964 & 1.64\end{array}$

\title{
Regression Equation
}
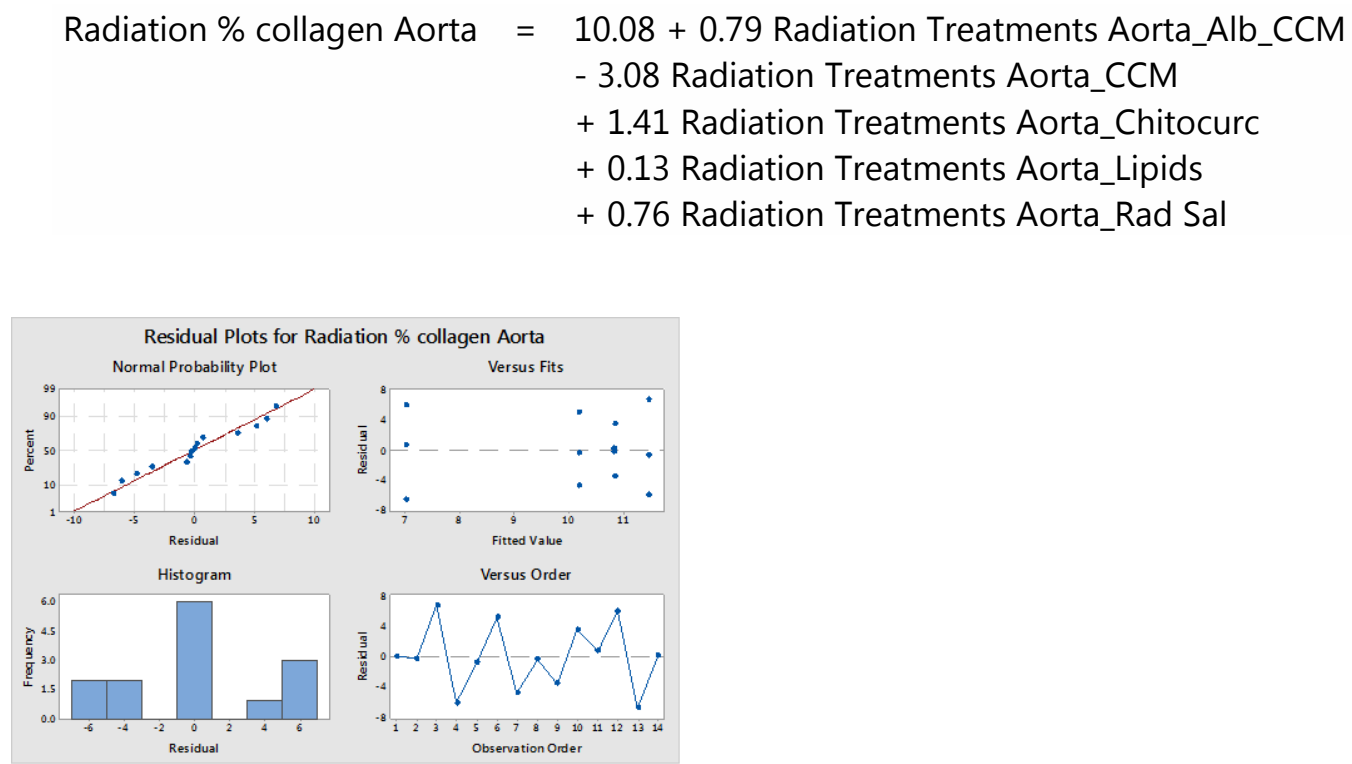

\section{Comparisons for Radiation \% collagen Aorta}

Tukey Pairwise Comparisons: Radiation Treatments Aorta

\author{
Grouping Information Using the Tukey Method and 95\% Confidence \\ Radiation \\ Treatments

\begin{tabular}{|c|c|c|c|}
\hline Aorta & $\mathrm{N}$ & Mean & Grouping \\
\hline Chitocurc & 3 & 11.4870 & A \\
\hline Alb_CCM & 2 & 10.8664 & A \\
\hline Rad Sal & 3 & 10.8390 & A \\
\hline Lipids & 3 & 10.2058 & A \\
\hline CCM & 3 & 7.0003 & A \\
\hline
\end{tabular} \\ Means that do not share a letter are significantly different.
}

\section{Tukey Simultaneous Tests for Differences of Means}

\begin{tabular}{|c|c|c|c|c|}
\hline & $\begin{array}{r}\text { Difference } \\
\text { of Means }\end{array}$ & $\begin{array}{r}\text { SE of } \\
\text { Difference }\end{array}$ & $\begin{array}{c}\text { Simultaneous } \\
95 \% \mathrm{CI}\end{array}$ & T-Value \\
\hline
\end{tabular}




$\begin{array}{lrrrrr}\text { CCM - Alb_CCM } & -3.87 & 4.73 & (-19.80,12.07) & -0.82 & 0.919 \\ \text { Chitocurc - Alb_CCM } & 0.62 & 4.73 & (-15.31,16.55) & 0.13 & 1.000 \\ \text { Lipids - Alb_CCM } & -0.66 & 4.73 & (-16.59,15.27) & -0.14 & 1.000 \\ \text { Rad Sal - Alb_CCM } & -0.03 & 4.73 & (-15.96,15.90) & -0.01 & 1.000 \\ \text { Chitocurc - CCM } & 4.49 & 4.23 & (-9.76,18.74) & 1.06 & 0.822 \\ \text { Lipids - CCM } & 3.21 & 4.23 & (-11.04,17.46) & 0.76 & 0.937 \\ \text { Rad Sal - CCM } & 3.84 & 4.23 & (-10.41,18.09) & 0.91 & 0.887 \\ \text { Lipids - Chitocurc } & -1.28 & 4.23 & (-15.53,12.97) & -0.30 & 0.998 \\ \text { Rad Sal - Chitocurc } & -0.65 & 4.23 & (-14.90,13.60) & -0.15 & 1.000 \\ \text { Rad Sal - Lipids } & 0.63 & 4.23 & (-13.62,14.88) & 0.15 & 1.000\end{array}$

Individual confidence level $=99.17 \%$

Right Ventricle

\section{General Linear Model: Radiation \% collagen RV versus ... eatments $\mathrm{RV}$}

\section{Method}

Factor coding $\quad(-1,0,+1)$

\section{Factor Information}

\begin{tabular}{llrl} 
Factor & Type & Levels & Values \\
\hline Radiation Treatments RV & Fixed & 5 Alb_CCM, CCM, Chitocurc, Lipids, Rad Sal
\end{tabular}

\section{Analysis of Variance}

\begin{tabular}{lrrrrr} 
Source & DF & Adj SS & Adj MS & F-Value & P-Value \\
\hline Radiation Treatments RV & 4 & 0.1234 & 0.03086 & 0.93 & 0.482 \\
Error & 10 & 0.3302 & 0.03302 & & \\
Total & 14 & 0.4537 & & &
\end{tabular}

\section{Model Summary}

\begin{tabular}{rrrr} 
S & R-sq & R-sq(adj) & R-sq(pred) \\
\hline 0.181727 & $27.21 \%$ & $0.00 \%$ & $0.00 \%$
\end{tabular}

\section{Coefficients}

\begin{tabular}{lrrrrr} 
Term & Coef & SE Coef & T-Value & P-Value & VIF \\
\hline $\begin{array}{l}\text { Constant } \\
\text { Radiation Treatments RV }\end{array}$ & 0.3978 & 0.0469 & 8.48 & 0.000 & \\
$\quad$ Alb_CCM & -0.0986 & 0.0938 & -1.05 & 0.318 & 1.60
\end{tabular}




$\begin{array}{lrrrrr}\text { CCM } & -0.0910 & 0.0938 & -0.97 & 0.355 & 1.60 \\ \text { Chitocurc } & 0.0607 & 0.0938 & 0.65 & 0.532 & 1.60 \\ \text { Lipids } & -0.0103 & 0.0938 & -0.11 & 0.915 & 1.60\end{array}$

\section{Regression Equation}

$$
\begin{aligned}
\text { Radiation \% collagen RV = } & 0.3978-0.0986 \text { Radiation Treatments RV_Alb_CCM } \\
& -0.0910 \text { Radiation Treatments RV_CCM } \\
& +0.0607 \text { Radiation Treatments RV_Chitocurc } \\
& -0.0103 \text { Radiation Treatments RV_Lipids } \\
& +0.1391 \text { Radiation Treatments RV_Rad Sal }
\end{aligned}
$$

Fits and Diagnostics for Unusual Observations

\begin{tabular}{rrrrrr}
\multicolumn{3}{c}{ Radiation \% } \\
Obs & collagen RV & Fit & Resid & Std Resid \\
\hline 5 & 0.077 & 0.459 & -0.382 & -2.57 & R \\
$R$ Large residual & & & & &
\end{tabular}

Residual Plots for Radiation \% collagen RV

\begin{tabular}{|c|c|c|c|}
\hline Radiation & & & \\
\hline Treatments RV & $\mathrm{N}$ & Mean & Grouping \\
\hline Rad Sal & 3 & 0.536940 & $A$ \\
\hline Chitocurc & 3 & 0.458523 & A \\
\hline Lipids & 3 & 0.387557 & $A$ \\
\hline
\end{tabular}

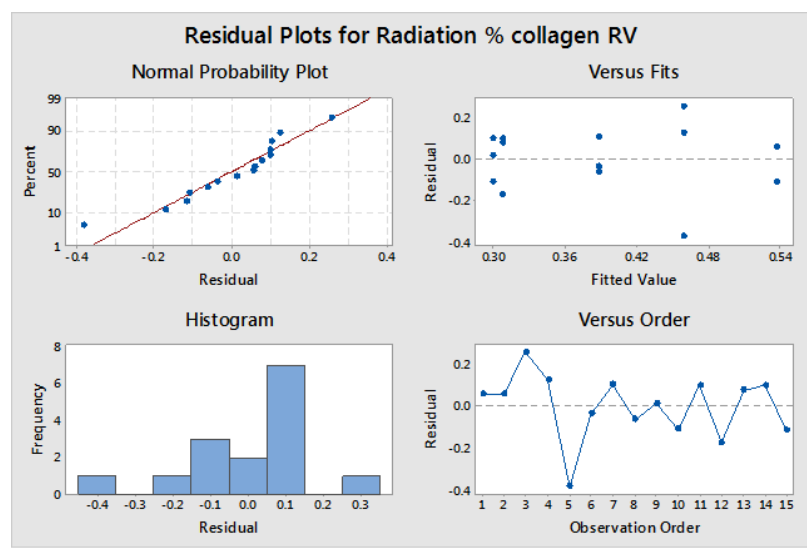

\section{Comparisons for Radiation \% collagen RV}

Tukey Pairwise Comparisons: Radiation Treatments RV

Grouping Information Using the Tukey Method and 95\% Confidence 


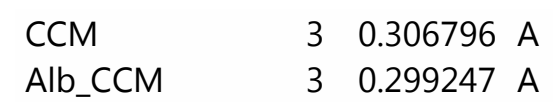

Means that do not share a letter are significantly different.

\section{Tukey Simultaneous Tests for Differences of Means}

\begin{tabular}{lrrcrr}
$\begin{array}{l}\text { Difference of Radiation } \\
\text { Treatments RV Levels }\end{array}$ & $\begin{array}{r}\text { Difference } \\
\text { of Means }\end{array}$ & $\begin{array}{r}\text { SE of } \\
\text { Difference }\end{array}$ & $\begin{array}{c}\text { Simultaneous } \\
95 \% \text { CI }\end{array}$ & $\begin{array}{r}\text { T-Value } \\
\text { P-Value }\end{array}$ \\
\hline CCM - Alb_CCM & 0.008 & 0.148 & $(-0.480,0.495)$ & 0.05 & 1.000 \\
Chitocurc - Alb_CCM & 0.159 & 0.148 & $(-0.329,0.647)$ & 1.07 & 0.816 \\
Lipids - Alb_CCM & 0.088 & 0.148 & $(-0.400,0.576)$ & 0.60 & 0.973 \\
Rad Sal - Alb_CCM & 0.238 & 0.148 & $(-0.250,0.726)$ & 1.60 & 0.528 \\
Chitocurc - CCM & 0.152 & 0.148 & $(-0.336,0.640)$ & 1.02 & 0.840 \\
Lipids - CCM & 0.081 & 0.148 & $(-0.407,0.569)$ & 0.54 & 0.980 \\
Rad Sal - CCM & 0.230 & 0.148 & $(-0.258,0.718)$ & 1.55 & 0.556 \\
Lipids - Chitocurc & -0.071 & 0.148 & $(-0.559,0.417)$ & -0.48 & 0.988 \\
Rad Sal - Chitocurc & 0.078 & 0.148 & $(-0.409,0.566)$ & 0.53 & 0.982 \\
Rad Sal - Lipids & 0.149 & 0.148 & $(-0.338,0.637)$ & 1.01 & 0.847
\end{tabular}

Individual confidence level $=99.18 \%$

Left Ventricle

\section{General Linear Model: Radiation \% collagen LV versus ... reatments $L V$}

\section{Method}

Factor coding $\quad(-1,0,+1)$

\section{Factor Information}

\begin{tabular}{llrl} 
Factor & Type & Levels & Values \\
\hline Radiation Treatments LV & Fixed & 5 Alb_CCM, CCM, Chitocurc, Lipids, Rad Sal
\end{tabular}

\section{Analysis of Variance}

\begin{tabular}{lrrrrr} 
Source & DF & Adj SS & Adj MS & F-Value & P-Value \\
\hline Radiation Treatments LV & 4 & 0.1182 & 0.02954 & 0.76 & 0.572 \\
Error & 10 & 0.3861 & 0.03861 & & \\
Total & 14 & 0.5043 & & &
\end{tabular}

\section{Model Summary}




\begin{tabular}{rrrr} 
S & R-sq & R-sq(adj) & R-sq(pred) \\
\hline 0.196501 & $23.43 \%$ & $0.00 \%$ & $0.00 \%$
\end{tabular}

Coefficients

\begin{tabular}{lrrrrr} 
Term & Coef & SE Coef & T-Value & P-Value & VIF \\
\hline Constant & 0.3080 & 0.0507 & 6.07 & 0.000 & \\
Radiation Treatments LV & & & & & \\
Alb_CCM & -0.147 & 0.101 & -1.45 & 0.177 & 1.60 \\
CCM & 0.116 & 0.101 & 1.15 & 0.278 & 1.60 \\
Chitocurc & 0.045 & 0.101 & 0.45 & 0.665 & 1.60 \\
Lipids & -0.039 & 0.101 & -0.38 & 0.712 & 1.60
\end{tabular}

\section{Regression Equation}

$$
\begin{aligned}
\text { Radiation \% collagen LV = } & 0.3080-0.147 \text { Radiation Treatments LV_Alb_CCM } \\
& +0.116 \text { Radiation Treatments LV_CCM } \\
& +0.045 \text { Radiation Treatments LV_Chitocurc } \\
& -0.039 \text { Radiation Treatments LV_Lipids } \\
& +0.024 \text { Radiation Treatments LV_Rad Sal }
\end{aligned}
$$

Residual Plots for Radiation \% collagen LV

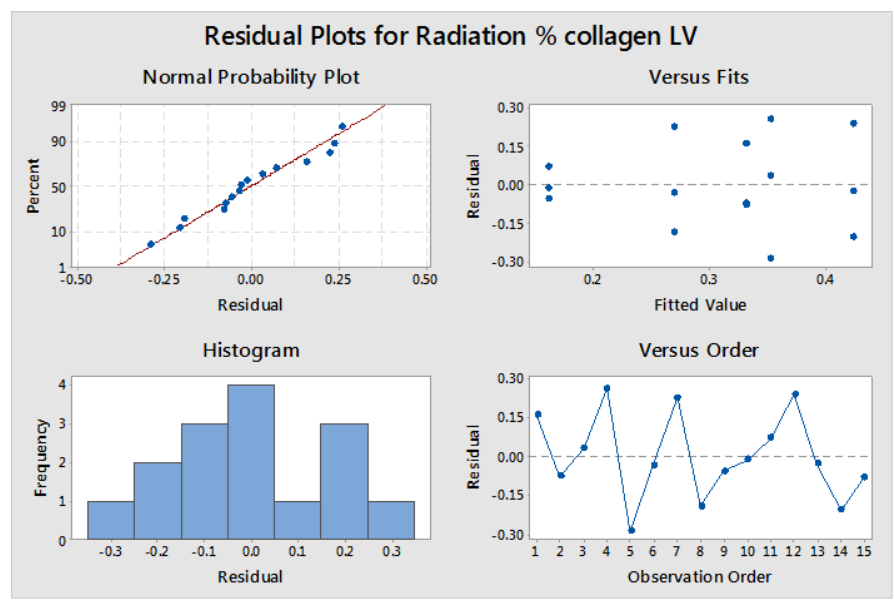

\section{Comparisons for Radiation \% collagen LV}

Tukey Pairwise Comparisons: Radiation Treatments LV

Grouping Information Using the Tukey Method and 95\% Confidence

Radiation

Treatments LV N Mean Grouping 


$\begin{array}{llll}\text { CCM } & 3 & 0.424469 & \text { A } \\ \text { Chitocurc } & 3 & 0.353318 & \text { A } \\ \text { Rad Sal } & 3 & 0.332048 & \text { A } \\ \text { Lipids } & 3 & 0.269450 & \text { A } \\ \text { Alb_CCM } & 3 & 0.160688 & \text { A }\end{array}$

Means that do not share a letter are significantly different.

Tukey Simultaneous Tests for Differences of Means

\begin{tabular}{lrrcrr}
$\begin{array}{l}\text { Difference of Radiation } \\
\text { Treatments LV Levels }\end{array}$ & $\begin{array}{r}\text { Difference } \\
\text { of Means }\end{array}$ & $\begin{array}{r}\text { SE of } \\
\text { Difference }\end{array}$ & $\begin{array}{c}\text { Simultaneous } \\
95 \% \text { CI }\end{array}$ & $\begin{array}{r}\text { T-Value } \\
\text { P-Value }\end{array}$ \\
\hline CCM - Alb_CCM & 0.264 & 0.160 & $(-0.264,0.791)$ & 1.64 & 0.505 \\
Chitocurc - Alb_CCM & 0.193 & 0.160 & $(-0.335,0.720)$ & 1.20 & 0.751 \\
Lipids - Alb_CCM & 0.109 & 0.160 & $(-0.419,0.636)$ & 0.68 & 0.957 \\
Rad Sal - Alb_CCM & 0.171 & 0.160 & $(-0.356,0.699)$ & 1.07 & 0.818 \\
Chitocurc - CCM & -0.071 & 0.160 & $(-0.599,0.456)$ & -0.44 & 0.991 \\
Lipids - CCM & -0.155 & 0.160 & $(-0.683,0.373)$ & -0.97 & 0.864 \\
Rad Sal - CCM & -0.092 & 0.160 & $(-0.620,0.435)$ & -0.58 & 0.976 \\
Lipids - Chitocurc & -0.084 & 0.160 & $(-0.611,0.444)$ & -0.52 & 0.983 \\
Rad Sal - Chitocurc & -0.021 & 0.160 & $(-0.549,0.506)$ & -0.13 & 1.000 \\
Rad Sal - Lipids & 0.063 & 0.160 & $(-0.465,0.590)$ & 0.39 & 0.994
\end{tabular}

Individual confidence level $=99.18 \%$

Immunohistology

Aorta

\section{General Linear Model: Radiation intensity Aorta versus ... ments Aorta}

\section{Method}

Factor coding $\quad(-1,0,+1)$

\section{Factor Information}

\begin{tabular}{llrl} 
Factor & Type & Levels Values \\
\hline Radiation Treatments Aorta & Fixed & 5 Alb/CCM, CCM, Chitocurc, Lipids, Rad Sal
\end{tabular}

\section{Analysis of Variance}

\begin{tabular}{lrrrrr} 
Source & DF & Adj SS & Adj MS & F-Value & P-Value \\
\hline Radiation Treatments Aorta & 4 & $5.79713 \mathrm{E}+13$ & $1.44928 \mathrm{E}+13$ & 0.35 & 0.832
\end{tabular}


$\begin{array}{lrrr}6 & 6 & 2.45015 \mathrm{E}+14 & 4.08359 \mathrm{E}+13\end{array}$

Total $10 \quad 3.02987 \mathrm{E}+14$

Model Summary

\begin{tabular}{rrrr} 
S & R-sq & R-sq(adj) & R-sq(pred) \\
\hline 6390297 & $19.13 \%$ & $0.00 \%$ & $0.00 \%$
\end{tabular}

\section{Coefficients}

\begin{tabular}{lrrrrr} 
Term & Coef & SE Coef & T-Value & P-Value & VIF \\
\hline Constant & 3327839 & 1952268 & 1.70 & 0.139 & \\
Radiation Treatments Aorta & & & & & \\
Alb/CCM & 487644 & 4007757 & 0.12 & 0.907 & 1.57 \\
CCM & -1166523 & 4007757 & -0.29 & 0.781 & 1.57 \\
Chitocurc & 3773903 & 3461001 & 1.09 & 0.317 & 1.44 \\
Lipids & -2517394 & 4007757 & -0.63 & 0.553 & 1.57
\end{tabular}

\section{Regression Equation}
Radiation intensity Aorta $=3327839+487644$ Radiation Treatments Aorta_Alb/CCM
- 1166523 Radiation Treatments Aorta_CCM
+ 3773903 Radiation Treatments Aorta_Chitocurc
- 2517394 Radiation Treatments Aorta_Lipids
- 577630 Radiation Treatments Aorta_Rad Sal

Fits and Diagnostics for Unusual Observations

\begin{tabular}{|c|c|c|c|c|}
\hline Obs & $\begin{array}{r}\text { Radiation } \\
\text { intensity } \\
\text { Aorta }\end{array}$ & Fit & Resid & $\begin{array}{r}\text { Std } \\
\text { Resid }\end{array}$ \\
\hline 3 & 18662829 & 7101742 & 11561087 & $2.22 \mathrm{R}$ \\
\hline
\end{tabular}

Residual Plots for Radiation intensity Aorta 


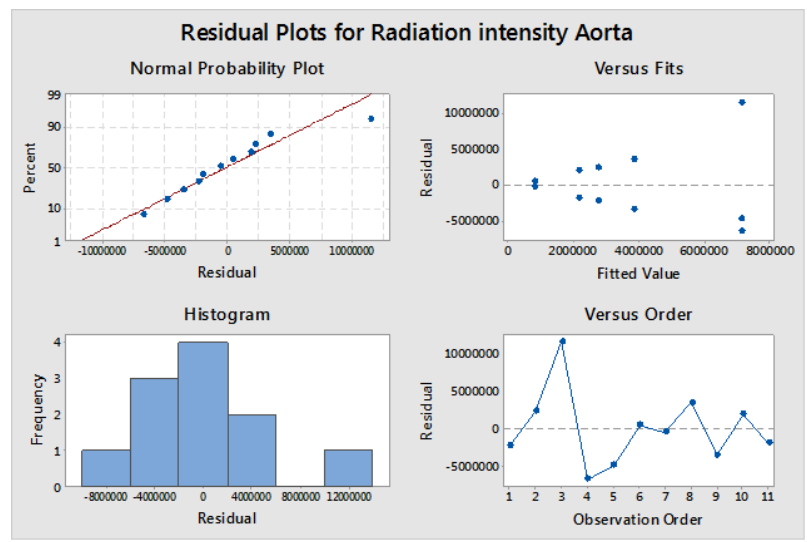

\title{
Comparisons for Radiation intensity Aorta
}

Tukey Pairwise Comparisons: Radiation Treatments Aorta

\author{
Grouping Information Using the Tukey Method and 80\% Confidence \\ Radiation \\ Treatments

\begin{tabular}{|c|c|c|c|}
\hline Aorta & $\mathrm{N}$ & Mean & Grouping \\
\hline Chitocurc & 3 & 7101742 & A \\
\hline Alb/CCM & 2 & 3815483 & $A$ \\
\hline Rad Sal & 2 & 2750209 & $A$ \\
\hline CCM & 2 & 2161316 & $A$ \\
\hline Lipids & 2 & 810446 & $A$ \\
\hline
\end{tabular} \\ Means that do not share a letter are significantly different.
}

\section{Tukey Simultaneous Tests for Differences of Means}

\begin{tabular}{|c|c|c|c|c|c|}
\hline $\begin{array}{l}\text { Difference of Radiation } \\
\text { Treatments Aorta } \\
\text { Levels }\end{array}$ & $\begin{array}{r}\text { Difference } \\
\text { of Means }\end{array}$ & $\begin{array}{r}\text { SE of } \\
\text { Difference }\end{array}$ & Simultaneous 80\% CI & T-Value & $\begin{array}{r}\text { Adjusted } \\
\text { P-Value }\end{array}$ \\
\hline CCM - Alb/CCM & -1654168 & 6390297 & $\begin{array}{c}(-17876021 \\
14567686)\end{array}$ & -0.26 & 0.999 \\
\hline Chitocurc - Alb/CCM & 3286259 & 5833516 & $\begin{array}{c}(-11522199 \\
18094717)\end{array}$ & 0.56 & 0.976 \\
\hline Lipids - Alb/CCM & -3005038 & 6390297 & $\begin{array}{c}(-19226891 \\
13216816)\end{array}$ & -0.47 & 0.987 \\
\hline Rad Sal - Alb/CCM & -1065274 & 6390297 & $\begin{array}{c}(-17287128 \\
15156579)\end{array}$ & -0.17 & 1.00 \\
\hline Chitocurc - CCM & 4940427 & 5833516 & $(-9868032,19748885)$ & 0.85 & 0.90 \\
\hline Lipids - CCM & -1350870 & 6390297 & $\begin{array}{c}(-17572723 \\
14870983)\end{array}$ & -0.21 & 0.99 \\
\hline
\end{tabular}




$\begin{array}{lrcccr}\text { Rad Sal - CCM } & 588893 & 6390297 & (-15632960, & 0.09 & 1.000 \\ & & & 16810747) & & \\ \text { Lipids - Chitocurc } & -6291297 & 5833516 & (-21099755,8517162) & -1.08 & 0.812 \\ \text { Rad Sal - Chitocurc } & -4351533 & 5833516 & (-19159992, & -0.75 & 0.937 \\ & & & 10456925) & & \\ \text { Rad Sal - Lipids } & 1939763 & 6390297 & (-14282090, & 0.30 & 0.998 \\ & & & 18161617) & & \end{array}$

Individual confidence level $=95.58 \%$

Right Ventricle

\section{General Linear Model: Radiation intensity RV versus ... Treatments RV}

\section{Method}

Factor coding $\quad(-1,0,+1)$

\section{Factor Information}

\begin{tabular}{llrl} 
Factor & Type & Levels & Values \\
\hline Radiation Treatments RV & Fixed & 5 Alb/CCM, CCM, Chitocurc, Lipids, Rad Sal
\end{tabular}

\section{Analysis of Variance}

\begin{tabular}{lrrrrr} 
Source & DF & Adj SS & Adj MS & F-Value & P-Value \\
\hline Radiation Treatments RV & 4 & $2.21318 \mathrm{E}+14$ & $5.53294 \mathrm{E}+13$ & 0.68 & 0.620 \\
Error & 9 & $7.27715 \mathrm{E}+14$ & $8.08572 \mathrm{E}+13$ & & \\
Total & 13 & $9.49033 \mathrm{E}+14$ & & &
\end{tabular}

\section{Model Summary}

\begin{tabular}{rrrr} 
S & R-sq & R-sq(adj) & R-sq(pred) \\
\hline 8992066 & $23.32 \%$ & $0.00 \%$ & $0.00 \%$
\end{tabular}

\section{Coefficients}

\begin{tabular}{lrrrrr} 
Term & Coef & SE Coef & T-Value & P-Value & VIF \\
\hline Constant & 15647420 & 2435063 & 6.43 & 0.000 & \\
Radiation Treatments RV & & & & & \\
Alb/CCM & -6378645 & 5494243 & -1.16 & 0.276 & 1.84 \\
CCM & 4745886 & 4701168 & 1.01 & 0.339 & 1.64 \\
Chitocurc & 4554662 & 4701168 & 0.97 & 0.358 & 1.64 \\
Lipids & -1660251 & 4701168 & -0.35 & 0.732 & 1.64
\end{tabular}




\section{Regression Equation}

Radiation intensity RV $=15647420-6378645$ Radiation Treatments RV_Alb/CCM

+ 4745886 Radiation Treatments RV_CCM

+ 4554662 Radiation Treatments RV_Chitocurc

- 1660251 Radiation Treatments RV_Lipids

- 1261652 Radiation Treatments RV_Rad Sal

Residual Plots for Radiation intensity RV

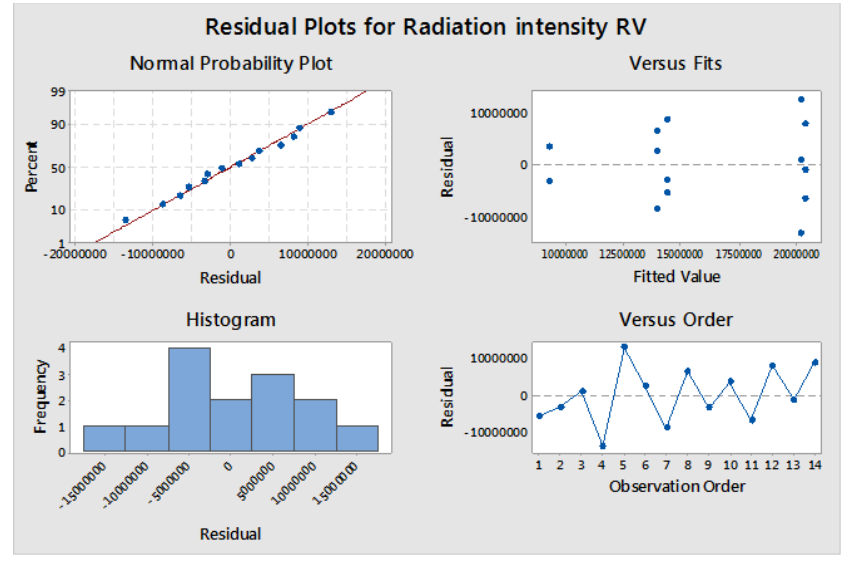

\section{Comparisons for Radiation intensity RV}

Tukey Pairwise Comparisons: Radiation Treatments RV

\section{Grouping Information Using the Tukey Method and 95\% Confidence}

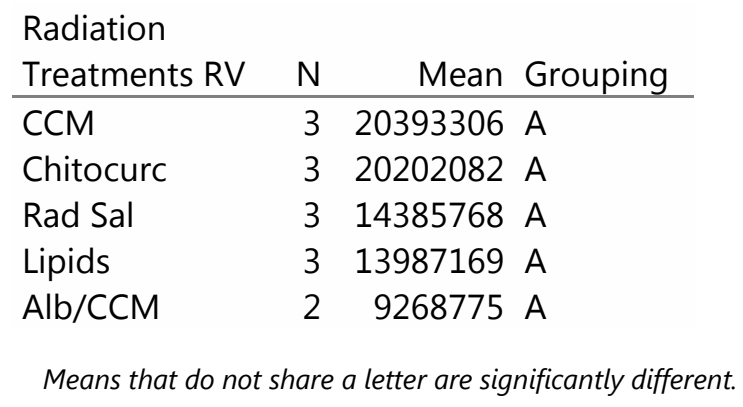

\section{Tukey Simultaneous Tests for Differences of Means}

\begin{tabular}{lrrcrr}
$\begin{array}{l}\text { Difference of Radiation } \\
\text { Treatments RV Levels }\end{array}$ & $\begin{array}{r}\text { Difference } \\
\text { of Means }\end{array}$ & $\begin{array}{r}\text { SE of } \\
\text { Difference }\end{array}$ & Simultaneous 95\% CI & $\begin{array}{r}\text { T-Value } \\
\text { Adjusted } \\
\text { P-Value }\end{array}$ \\
\hline CCM - Alb/CCM & 11124531 & 8208595 & $\begin{array}{c}(-16504191, \\
38753253)\end{array}$ & 1.36 & 0.667
\end{tabular}




$\begin{array}{lccccc}\text { Chitocurc - Alb/CCM } & 10933307 & 8208595 & \begin{array}{c}(-16695415, \\ 38562029)\end{array} & 1.33 & 0.680 \\ \text { Lipids - Alb/CCM } & 4718394 & 8208595 & \begin{array}{c}(-22910328, \\ 32347116)\end{array} & 0.57 & 0.976 \\ \text { Rad Sal - Alb/CCM } & 5116993 & 8208595 & \begin{array}{c}(-22511729, \\ 32745715)\end{array} & 0.62 & 0.967 \\ \text { Chitocurc - CCM } & -191224 & 7341991 & \begin{array}{c}(-24903104, \\ 24520656)\end{array} & -0.03 & 1.000 \\ \text { Lipids - CCM } & -6406137 & 7341991 & \begin{array}{c}(-31118017, \\ 18305743)\end{array} & -0.87 & 0.900 \\ \text { Rad Sal - CCM } & -6007538 & 7341991 & \begin{array}{c}(-30719418, \\ 18704343)\end{array} & -0.82 & 0.918 \\ \text { Lipids - Chitocurc } & -6214913 & 7341991 & \begin{array}{c}(-30926793, \\ 18496967)\end{array} & -0.85 & 0.909 \\ \text { Rad Sal - Chitocurc } & -5816314 & 7341991 & \begin{array}{c}(-30528194, \\ 18895567)\end{array} & -0.79 & 0.927 \\ \text { Rad Sal - Lipids } & 398599 & 7341991 & \begin{array}{c}(-24313281, \\ 25110480)\end{array} & 0.05 & 1.000\end{array}$

Individual confidence level $=99.17 \%$

Left Ventricle

\section{General Linear Model: Radiation intensity LV versus ... Treatments LV}

\section{Method}

Factor coding $(-1,0,+1)$

\section{Factor Information}

\begin{tabular}{llrl} 
Factor & Type & Levels & Values \\
\hline Radiation Treatments LV & Fixed & 5 Alb/CCM, CCM, Chitocurc, Lipids, Rad Sal
\end{tabular}

\section{Analysis of Variance}

\begin{tabular}{lrrrrr} 
Source & DF & Adj SS & Adj MS & F-Value & P-Value \\
\hline Radiation Treatments LV & 4 & $9.96928 \mathrm{E}+13$ & $2.49232 \mathrm{E}+13$ & 0.26 & 0.894 \\
Error & 9 & $8.52235 \mathrm{E}+14$ & $9.46927 \mathrm{E}+13$ & & \\
Total & 13 & $9.51927 \mathrm{E}+14$ & & &
\end{tabular}

\section{Model Summary}

\begin{tabular}{rrrr} 
S & R-sq & R-sq(adj) & R-sq(pred) \\
\hline 9731019 & $10.47 \%$ & $0.00 \%$ & $0.00 \%$
\end{tabular}




\section{Coefficients}

\begin{tabular}{lrrrrr} 
Term & Coef & SE Coef & T-Value & P-Value & VIF \\
\hline Constant & 17239085 & 2635173 & 6.54 & 0.000 & \\
Radiation Treatments LV & & & & & \\
$\quad$ Alb/CCM & -5791102 & 5945751 & -0.97 & 0.356 & 1.84 \\
CCM & 1399552 & 5087503 & 0.28 & 0.789 & 1.64 \\
Chitocurc & 433044 & 5087503 & 0.09 & 0.934 & 1.64 \\
Lipids & 2898589 & 5087503 & 0.57 & 0.583 & 1.64
\end{tabular}

\section{Regression Equation}

$$
\begin{aligned}
\text { Radiation intensity LV }= & 17239085-5791102 \text { Radiation Treatments LV_Alb/CCM } \\
& +1399552 \text { Radiation Treatments LV_CCM } \\
& +433044 \text { Radiation Treatments LV_Chitocurc } \\
& +2898589 \text { Radiation Treatments LV_Lipids } \\
& +1059917 \text { Radiation Treatments LV_Rad Sal }
\end{aligned}
$$

\begin{tabular}{|c|c|c|c|}
\hline $\begin{array}{l}\text { Radiation } \\
\text { Treatments LV }\end{array}$ & $\mathrm{N}$ & Mean & Grouping \\
\hline Lipids & 3 & 20137674 & $A$ \\
\hline CCM & 3 & 18638637 & A \\
\hline Rad Sal & 3 & 18299003 & $A$ \\
\hline Chitocurc & 3 & 17672130 & A \\
\hline Alb/CCM & 2 & 11447984 & A \\
\hline
\end{tabular}

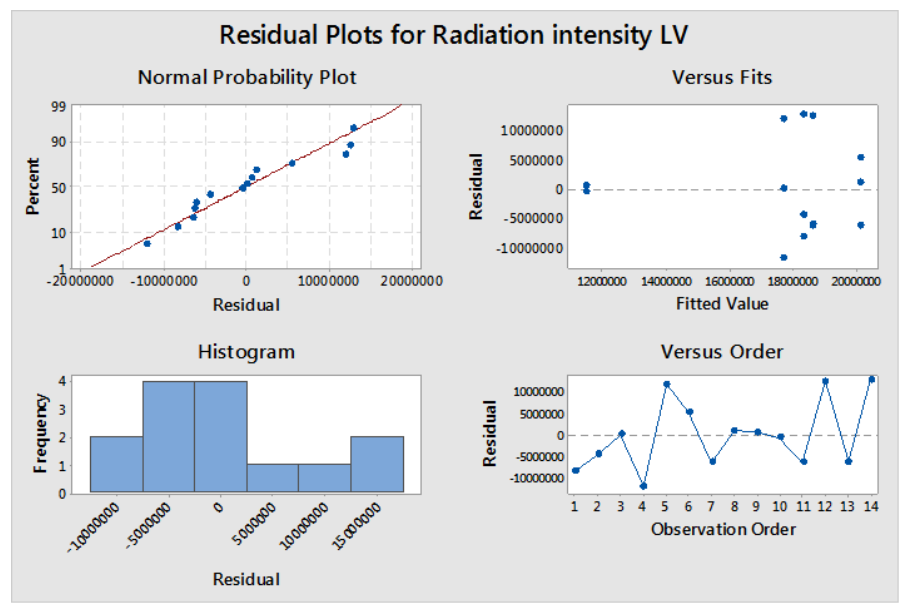

\section{Comparisons for Radiation intensity LV}

\section{Tukey Pairwise Comparisons: Radiation Treatments LV}

\section{Grouping Information Using the Tukey Method and 95\% Confidence}


Means that do not share a letter are significantly different.

\section{Tukey Simultaneous Tests for Differences of Means}

\begin{tabular}{lrrrrr}
$\begin{array}{l}\text { Difference of Radiation } \\
\text { Treatments LV Levels }\end{array}$ & $\begin{array}{r}\text { Difference } \\
\text { of Means }\end{array}$ & $\begin{array}{r}\text { SE of } \\
\text { Difference }\end{array}$ & Simultaneous 95\% CI & T-Value & $\begin{array}{r}\text { Adjusted } \\
\text { P-Value }\end{array}$ \\
\hline CCM - Alb/CCM & 7190653 & 8883165 & $\begin{array}{c}(-22708553, \\
37089860)\end{array}$ & 0.81 & 0.921 \\
Chitocurc - Alb/CCM & 6224146 & 8883165 & $\begin{array}{c}(-23675061, \\
36123353)\end{array}$ & 0.70 & 0.951 \\
Lipids - Alb/CCM & 8689690 & 8883165 & $\begin{array}{c}(-21209517, \\
38588897)\end{array}$ & 0.98 & 0.859 \\
Rad Sal - Alb/CCM & 6851019 & 8883165 & $\begin{array}{c}(-23048188, \\
36750226)\end{array}$ & 0.77 & 0.933 \\
Chitocurc - CCM & -966507 & 7945344 & $\begin{array}{c}(-27709171, \\
25776156)\end{array}$ & -0.12 & 1.000 \\
Lipids - CCM & 1499037 & 7945344 & $\begin{array}{c}(-25243627, \\
28241701)\end{array}$ & 0.19 & 1.000 \\
Rad Sal - CCM & -339634 & 7945344 & $\begin{array}{c}(-27082298, \\
26403029) \\
(-24277119,\end{array}$ & -0.04 & 1.000 \\
Lipids - Chitocurc & 2465544 & 7945344 & $29208208)$ & 0.31 & 0.998 \\
Rad Sal - Chitocurc & 626873 & 7945344 & $\begin{array}{c}(-26115790, \\
27369537)\end{array}$ & 0.08 & 1.000 \\
Rad Sal - Lipids & -1838671 & 7945344 & $\begin{array}{c}(-28581335, \\
24903993)\end{array}$ & -0.23 & 0.999 \\
& & & & &
\end{tabular}

Individual confidence level $=99.17 \%$

\begin{tabular}{|l|l|l|l|}
\hline ANOVA summaries & Aorta & Right Ventricle & Left Ventricle \\
\hline Aortic Outgrowth & 0.160 & NA & NA \\
\hline ROS Assay & 0.719 & NA & NA \\
\hline H and E & 0.454 & 0.997 & 0.347 \\
\hline Thickness & 0.501 & 0.497 & $<0.001$ \\
\hline Massons Trichrome & 0.840 & 0.482 & 0.572 \\
\hline Immunohistology & 0.832 & 0.620 & 0.894 \\
\hline
\end{tabular}

